\title{
Development and Applications of Transesterification Reactions Catalyzed by N-Heterocyclic Olefins
}

Marcus Blümel, ${ }^{[\mathrm{a}, \mathrm{b}]}$ Janina-Miriam Noy, ${ }^{[\mathrm{a}]}$ Dieter Enders, ${ }^{[\mathrm{b}]}$ Martina H. Stenzel, ${ }^{[\mathrm{a}]}$ and Thanh V. Nguyen*[a]

[a] School of Chemistry, University of New South Wales, Sydney, NSW 2052, Australia. *E-mail: t.v.nguyen@unsw.edu.au [b] Institute of Organic Chemistry, RWTH Aachen University, Landoltweg 1, 52074 Aachen, Germany

\section{Table of Contents}

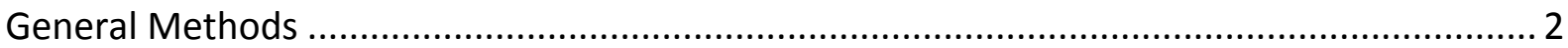

General Procedure A for the transesterification reaction of methyl esters with NHOs .......... 8

General procedure B for the transesterification reaction of vinyl esters with $\mathrm{NHOs} \mathrm{..............} 8$

General procedure $\mathrm{C}$ for the transesterification reaction with in-situ NHO formation ............ 8

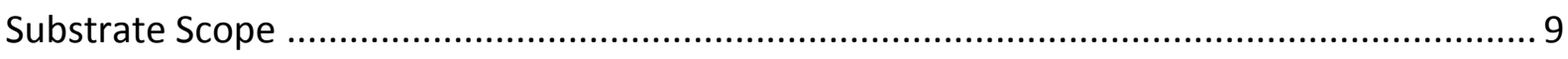

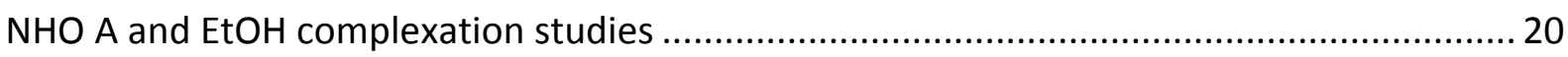

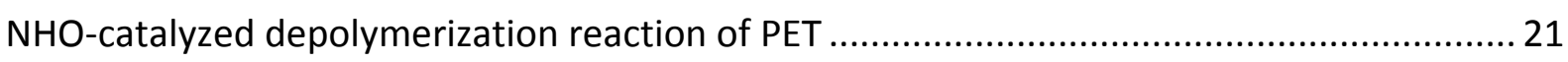

Synthesis of polyesters via an NHO-catalyzed transesterification reaction ........................... 22

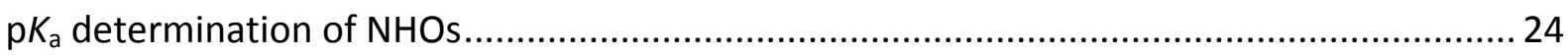

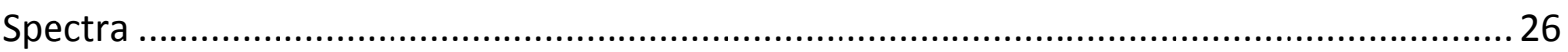




\section{General Methods}

Reactions, unless otherwise stated, were conducted under a positive pressure of dry nitrogen in oven-dried glassware. Toluene, hexane, dichloromethane (DCM), tetrahydrofuran (THF), and diethyl ether were dried with an SPS apparatus. Commercially available reagents were used as purchased unless otherwise noted. Analytical thin layer chromatography was performed using silica gel plates precoated with silica gel $60 \mathrm{~F}_{254}(0.2 \mathrm{~mm})$. Flash chromatography employed 230-400 mesh silica gel. Solvents used for chromatography are quoted as volume/volume ratios.

NMR spectroscopy was performed at $298 \mathrm{~K}$ using either a Bruker Avance III 300 (300.13 $\mathrm{MHz},{ }^{1} \mathrm{H} ; 75.5 \mathrm{MHz},{ }^{13} \mathrm{C}$; BBFO probe), a Avance I $300\left(300.13 \mathrm{MHz},{ }^{1} \mathrm{H} ; 75.5 \mathrm{MHz},{ }^{13} \mathrm{C}\right.$; BBFO probe), or a Avance III $400\left(400.13 \mathrm{MHz},{ }^{1} \mathrm{H} ; 100.6 \mathrm{MHz},{ }^{13} \mathrm{C}\right.$; BBFO probe or Prodigy cryoprobe) at the Mark Wainwright Analytical Centre at the University of New South Wales and using either a Varian Mercury $300\left(300.03 \mathrm{MHz},{ }^{1} \mathrm{H}\right)$, a Varian Inova 400 (399.97 $\left.\mathrm{MHz},{ }^{1} \mathrm{H}\right)$, or a Varian Inova $600\left(599.86 \mathrm{MHz},{ }^{1} \mathrm{H} ; 150.85 \mathrm{MHz},{ }^{13} \mathrm{C}\right)$ at the RWTH Aachen University. Data is expressed in parts per million (ppm) downfield shift from tetramethylsilane with residual solvent as an internal reference $(\delta 7.26 \mathrm{ppm}$ for chloroform, $5.27 \mathrm{ppm}$ for dichloromethane, $1.94 \mathrm{ppm}$ for acetonitrile, and $2.09 \mathrm{ppm}$ for the toluene methyl group) and is reported as position ( $\delta$ in ppm), multiplicity ( $\mathrm{s}=$ singlet, $\mathrm{d}=$ doublet, $\mathrm{t}=$ triplet, $\mathrm{q}=$ quartet, quint. $=$ quintet, $\mathrm{sxt} .=$ sextet, $\mathrm{spt} .=$ septet, $\mathrm{m}=$ multiplet $)$, coupling constant $(J$ in $\mathrm{Hz}$ ) and integration (number of protons). ${ }^{13} \mathrm{C}$ NMR spectra were recorded at $298 \mathrm{~K}$ with complete proton decoupling. Data is expressed in parts per million (ppm) downfield shift relative to the internal reference ( $\delta 77.2 \mathrm{ppm}$ for the central peak of deuterated chloroform).

Infrared spectra were obtained on a ThermoNicolet Avatar 370 FT-IR spectrometer and are reported in wavenumbers $\left(\mathrm{cm}^{-1}\right)$. HRMS were performed at the Bioanalytical Mass Spectrometry Facility within the Mark Wainwright Analytical Centre at the University of New South Wales on an Orbitrap LTQ XL (Thermo Fisher Scientific, San Jose, CA, USA) ion trap mass spectrometer. 


\section{Catalyst syntheses}<smiles>Cc1ncn(C)c1C</smiles><smiles>CCOCC</smiles><smiles></smiles>

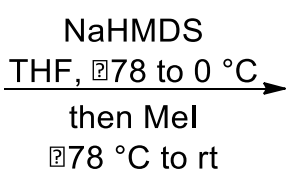<smiles></smiles>

The tetramethyl imidazolium intermediate was synthesized according to a modified literature procedure of Fürstner. ${ }^{1} 6.6 \mathrm{~mL}(15.0 \mathrm{~g}, 106.0 \mathrm{mmol}, 1.5$ equiv) methyl iodide was slowly added to a solution of $7.8 \mathrm{~g}$ (70.8 mmol, 1.0 equiv) 1,4,5-trimethyl imidazole in $90 \mathrm{~mL}$ dry ether and the reaction mixture was stirred overnight at ambient temperature. The resulting white suspension was filtered, the filter cake washed with dry ether $(3 \mathrm{x}, 50 \mathrm{~mL})$, and subsequently dried in vacuum to yield $17.66 \mathrm{~g}$ (70.0 mmol, 99\%) 1,3,4,5-tetramethyl imidazolium iodide as colorless powder.

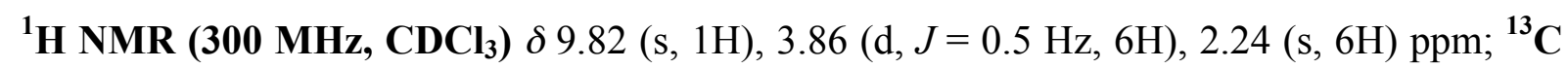
NMR (75 MHz, $\left.\mathbf{C D C l}_{3}\right) \delta 135.58,127.16,34.38,8.82$ ppm.

A suspension of $2.52 \mathrm{~g}$ (10.0 mmol, 1.0 equiv) 1,3,4,5-tetramethyl imidazolium iodide in 50 $\mathrm{mL}$ dry $\mathrm{THF}$ under nitrogen atmosphere at $-78{ }^{\circ} \mathrm{C}$ was slowly treated with $10.2 \mathrm{~mL}(10.2$ mmol, 1.0 equiv) NaHMDS-solution ( $1 \mathrm{M}$ in THF), stirred for $10 \mathrm{~min}$ at $-78{ }^{\circ} \mathrm{C}$ and then slowly warmed to $0{ }^{\circ} \mathrm{C}$. Subsequently, the reaction mixture was cooled to $-78^{\circ} \mathrm{C}$ and $1.0 \mathrm{~mL}$ (16.1 mmol, 1.6 equiv) methyl iodide was added dropwise. The reaction mixture was slowly warmed to ambient temperature and continued overnight. After the addition of $50 \mathrm{~mL}$ dry ether, the resulting suspension was filtered and the filter cake washed with dry ether $(50 \mathrm{~mL})$. Dissolving the filter cake in dry dichloromethane, a second filtration, and removal of the volatiles under reduced pressure gave the crude product, which was recrystallized from hot chloroform to yield $2.52 \mathrm{~g}(9.47 \mathrm{mmol}, 95 \%)$ pre-A as off-white needles. ${ }^{2}$

${ }^{1}$ H NMR (300 MHz, CDCl $) \delta 3.74(\mathrm{~s}, 6 \mathrm{H}), 2.82(\mathrm{~s}, 3 \mathrm{H}), 2.24(\mathrm{~s}, 6 \mathrm{H}) \mathrm{ppm} ;{ }^{13} \mathbf{C}$ NMR (75 $\left.\mathbf{M H z}, \mathbf{C D C l}_{3}\right) \delta 142.9,125.9,33.3,12.7,9.3 \mathrm{ppm}$.

\footnotetext{
${ }^{1}$ A. Fürstner, M. Alcarazo, R. Goddard, C. W. Lehmann, Angew. Chemie Int. Ed. 2008, 47, 3210-3214.

${ }^{2}$ T. Peppel, C. Roth, K. Fumino, D. Paschek, M. Köckerling, R. Ludwig, Angew. Chemie Int. Ed. 2011, 50, 6661-6665.
} 

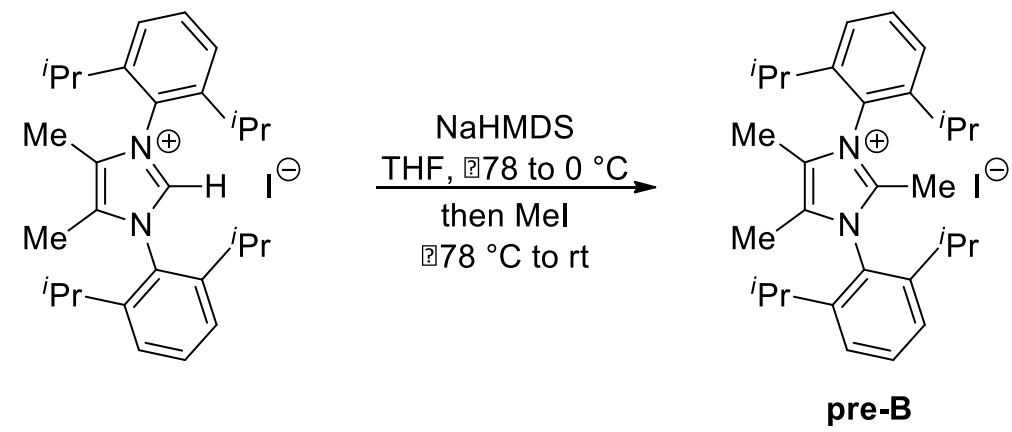

Under argon $25 \mathrm{~mL}$ dry THF were added to $2.05 \mathrm{~g}$ (4.52 mmol, 1.0 equiv) 1,3-bis(2,6-diisopropylphenyl)-4,5-dimethyl-1 $H$-imidazol-3-ium chloride and the resulting suspension treated dropwise with $4.6 \mathrm{~mL}$ (4.6 mmol, 1.0 equiv) NaHMDS-solution $\left(1 \mathrm{M}\right.$ in THF) at $-78{ }^{\circ} \mathrm{C}$. After slowly warming to $0{ }^{\circ} \mathrm{C}$ over 2 hours and cooling to $-78{ }^{\circ} \mathrm{C} 0.42 \mathrm{~mL}(0.96 \mathrm{~g}, 6.75$ mmol, 1.5 equiv) methyl iodide were slowly added and the reaction mixture gradually warmed to room temperature overnight. $30 \mathrm{~mL}$ dry ether were added, the mixture filtered, and the filter cake washed with dry ether $(3 \mathrm{x}, 20 \mathrm{~mL})$. Subsequently the filter cake was dissolved in dry dichloromethane, filtered again, and the filtrate concentrated under reduced pressure to yield $2.39 \mathrm{~g}$ (4.28 mmol, 95\%) 2-methyl imidazolium iodide pre-B.

${ }^{1}$ H NMR (300 MHz, CD ${ }_{3}$ CN) $\delta 7.66-7.74(\mathrm{~m}, 2 \mathrm{H}), 7.50-7.57$ (m, 4H), 2.34 (spt, $J=6.8$ $\mathrm{Hz}, 4 \mathrm{H}), 2.02-2.09$ (m, 9H), 1.25 (d, $J=6.8 \mathrm{~Hz}, 12 \mathrm{H}), 1.16(\mathrm{~d}, J=6.9 \mathrm{~Hz}, 12 \mathrm{H}) \mathrm{ppm} ;{ }^{13} \mathbf{C}$ NMR (75 MHz, CD ${ }_{3}$ CN) $\delta 145.3,143.3,132.8,128.8,127.0,125.8,29.1,24.2,24.2,12.2$, 10.4 ppm; IR: 3150, 3068, 2960, 2939, 1630, 1602, 1536, $1468 \mathrm{~cm}^{-1}$; ESI-MS m/z: 431.3 $\left([\mathrm{M}-\mathrm{I}]^{+}, 100 \%\right)$; Anal. Calcd. for $\mathrm{C}_{30} \mathrm{H}_{43} \mathrm{~N}_{2}{ }^{+}: m / z=431.3421$; found 431.3422 . 


\section{Optimization}

Table S1. Solvent Screening and optimization of NHO-catalyzed transesterification reaction. ${ }^{[\text {ad }}$

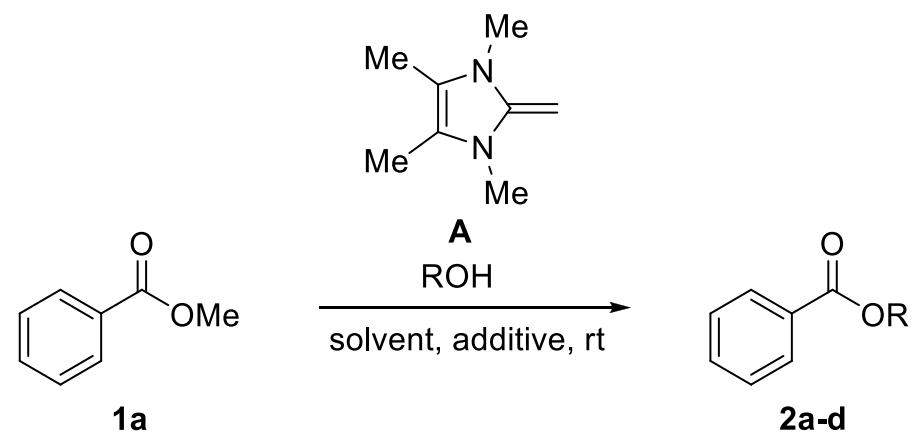

\begin{tabular}{|c|c|c|c|c|c|c|}
\hline entry & $\mathrm{R}$ & $\mathrm{ROH}$ [equiv] & solvent & $\mathbf{A}[\mathrm{mol} \%]$ & $\operatorname{additive~}^{[\mathrm{b}]}$ & yield $[\%]^{[\mathrm{c}]}$ \\
\hline 1 & Et & 20 & - & 5 & - & 58 \\
\hline 2 & Et & 10 & - & 5 & - & 54 \\
\hline 3 & $\mathrm{Et}$ & 5 & - & 5 & - & 49 \\
\hline 4 & $\mathrm{Et}$ & 5 & $\mathrm{THF}$ & 5 & - & 61 \\
\hline 5 & $\mathrm{Et}$ & 5 & $\mathrm{CH}_{2} \mathrm{Cl}_{2}$ & 5 & - & 62 \\
\hline 6 & Et & 5 & Toluene & 5 & - & 41 \\
\hline 7 & $\mathrm{Et}$ & 5 & $\mathrm{THF}$ & 5 & $4 \AA \mathrm{MS}$ & 80 \\
\hline 8 & $\mathrm{Et}$ & 5 & $\mathrm{CH}_{2} \mathrm{Cl}_{2}$ & 5 & $4 \AA \mathrm{MS}$ & 80 \\
\hline 9 & ${ }^{n} \mathrm{Bu}$ & 5 & $\mathrm{THF}$ & 5 & $4 \AA \mathrm{MS}$ & 85 \\
\hline 10 & ${ }^{n} \mathrm{Bu}$ & 5 & $\mathrm{CH}_{2} \mathrm{Cl}_{2}$ & 5 & $4 \AA \mathrm{MS}$ & 79 \\
\hline 11 & ${ }^{n}$ Oct & 5 & $\mathrm{THF}$ & 5 & $4 \AA \mathrm{MS}$ & 86 \\
\hline 12 & ${ }^{n} \mathrm{Oct}$ & 5 & $\mathrm{CH}_{2} \mathrm{Cl}_{2}$ & 5 & $4 \AA ̊ \mathrm{MS}$ & 85 \\
\hline 13 & $\mathrm{Bn}$ & 5 & THF & 5 & $4 \AA \mathrm{MS}$ & 90 \\
\hline 14 & $\mathrm{Bn}$ & 5 & $\mathrm{CH}_{2} \mathrm{Cl}_{2}$ & 5 & $4 \AA \mathrm{MS}$ & 86 \\
\hline 15 & Et & 5 & $\mathrm{THF}$ & 2 & $4 \AA ̊ \mathrm{MS}$ & 69 \\
\hline 16 & Et & 5 & $\mathrm{THF}$ & 1 & $4 \AA ̊ M S$ & 62 \\
\hline
\end{tabular}

[a] The reactions were carried out with $1.0 \mathrm{mmol}$ methyl benzoate 1a and the alcohol in the presence of a catalytic amount of NHO $\mathbf{A}$ in $1 \mathrm{~mL}$ of the solvent $(c=1 \mathrm{M})$ at ambient temperature until the TLC indicated no further change in the reaction mixture. [b] $0.5 \mathrm{~g}$ of activated $4 \AA \mathrm{MS}$ was used. [c] Yield of the isolated products. 
Table S2. Effect of the catalyst and the substrate structure. ${ }^{[\mathrm{a}]}$<smiles>[R7]OC(=O)c1ccc([R])cc1</smiles>

A<smiles>[R10]OC(=O)c1ccc([R10])cc1</smiles><smiles>C=C1N(C(=O)c2ccccc2)C(C)=C(C)N1C(=O)c1ccccc1</smiles>

B

\begin{tabular}{|c|c|c|c|c|c|c|}
\hline entry & $\mathrm{R}^{1}$ & $\mathrm{R}^{2}$ & $\mathrm{R}^{3}$ & catalyst & $t[\mathrm{~h}]$ & yield $[\%]^{[b]}$ \\
\hline $1^{[\mathrm{c}]}$ & $\mathrm{H}$ & $\mathrm{Me}$ & Et & $\begin{array}{l}\mathbf{A} \\
\mathbf{B}\end{array}$ & $\begin{array}{l}2 \\
2\end{array}$ & $\begin{array}{l}80 \\
83\end{array}$ \\
\hline 2 & $\mathrm{H}$ & vinyl & Et & $\begin{array}{l}\mathbf{A} \\
\mathbf{B}\end{array}$ & $\begin{array}{c}1.5 \\
2\end{array}$ & $\begin{array}{l}90 \\
88\end{array}$ \\
\hline $3^{[\mathrm{cc}]}$ & $\mathrm{H}$ & $\mathrm{Me}$ & $n \mathrm{Bu}$ & $\begin{array}{l}\mathbf{A} \\
\mathbf{B}\end{array}$ & $\begin{array}{l}3 \\
3\end{array}$ & $\begin{array}{l}85 \\
84\end{array}$ \\
\hline 4 & $\mathrm{H}$ & vinyl & $n \mathrm{Bu}$ & $\begin{array}{l}\mathbf{A} \\
\mathbf{B}\end{array}$ & $\begin{array}{c}1.5 \\
2\end{array}$ & $\begin{array}{l}88 \\
85\end{array}$ \\
\hline $5^{[\mathrm{cc}]}$ & $\mathrm{H}$ & $\mathrm{Me}$ & $\mathrm{Bn}$ & $\begin{array}{l}\mathbf{A} \\
\mathbf{B}\end{array}$ & $\begin{array}{c}1.5 \\
4\end{array}$ & $\begin{array}{l}90 \\
88\end{array}$ \\
\hline 6 & $\mathrm{H}$ & vinyl & $\mathrm{Bn}$ & $\begin{array}{l}\mathbf{A} \\
\mathbf{B}\end{array}$ & $\begin{array}{l}1 \\
3\end{array}$ & $\begin{array}{l}91 \\
92\end{array}$ \\
\hline $7^{[\mathrm{c}]}$ & $\mathrm{OMe}$ & $\mathrm{Me}$ & ${ }^{n} \mathrm{Bu}$ & $\mathbf{A}$ & 3 & 81 \\
\hline $8^{[\mathrm{cc}]}$ & $\mathrm{OMe}$ & $\mathrm{Me}$ & ${ }^{i} \mathrm{Pr}$ & $\mathbf{A}$ & 12 & 29 \\
\hline $9^{[\mathrm{cc}]}$ & $\mathrm{OMe}$ & $\mathrm{Me}$ & ${ }^{t} \mathrm{Bu}$ & $\mathbf{A}$ & 24 & n.r. ${ }^{[d]}$ \\
\hline
\end{tabular}

[a] The reactions were carried out with $1.0 \mathrm{mmol}$ ester 1 and $5.0 \mathrm{mmol}$ alcohol in the presence of $5 \mathrm{~mol} \%$ catalyst in $1 \mathrm{~mL}$ THF $(c=1 \mathrm{M})$ at ambient temperature until the TLC indicated no further change in the reaction mixture. [b] Yield of the isolated product. [c] $0.5 \mathrm{~g} 4 \AA \mathrm{MS}$ was also added. [d] No reaction. Dipp $=2,6-\left({ }^{i} \operatorname{Pr}\right)_{2}{ }^{-}$ $\mathrm{C}_{6} \mathrm{H}_{3}$. 
Table S3. Transesterification reaction with the in-situ formed NHO.$^{[a]}$<smiles>COC(=O)c1ccccc1</smiles>

1a<smiles>C=C1N(C)C(C)=C(C)N1C</smiles>

A<smiles>[R]OC(C)(C)F</smiles>

THF, $4 \AA$ MS, rt<smiles></smiles><smiles>[R]OC(=O)c1ccccc1</smiles>

$2 a, b, d$

(6 mol \% pre-A $\left.+5 \mathrm{~mol} \% \mathrm{KO}^{t} \mathrm{Bu}\right)$

\begin{tabular}{|c|c|c|c|c|}
\hline entry & $\mathrm{R}^{1}$ & catalyst & $t[\mathrm{~h}]$ & yield $[\%]^{[\mathrm{b}]}$ \\
\hline 1 & Et & $\begin{array}{l}\mathbf{A} \\
\mathbf{C}\end{array}$ & $\begin{array}{l}2 \\
2\end{array}$ & $\begin{array}{l}80 \\
84\end{array}$ \\
\hline 2 & ${ }^{n} \mathrm{Bu}$ & $\begin{array}{l}\mathbf{A} \\
\mathbf{C}\end{array}$ & $\begin{array}{l}3 \\
3\end{array}$ & $\begin{array}{l}85 \\
86\end{array}$ \\
\hline 3 & $\mathrm{Bn}$ & $\begin{array}{l}\mathbf{A} \\
\mathbf{C}\end{array}$ & $\begin{array}{c}1.5 \\
2\end{array}$ & $\begin{array}{l}90 \\
91\end{array}$ \\
\hline
\end{tabular}

[a] The reactions were carried out with $1.0 \mathrm{mmol}$ ester $1 \mathrm{a}$ and $5.0 \mathrm{mmol}$ alcohol in the presence of $5 \mathrm{~mol} \%$ of catalyst in $1 \mathrm{~mL}$ THF $(c=1 \mathrm{M})$ at ambient temperature until the TLC indicated no further change in the reaction mixture. [b] Yield of the isolated product. 


\section{General Procedure A for the transesterification reaction of methyl esters with NHOs}

A suspension of $0.05 \mathrm{mmol}(5 \mathrm{~mol} \%)$ NHO azolium salt pre-A and $0.05 \mathrm{mmol}(5 \mathrm{~mol} \%)$ potassium tert-butoxide in $0.2 \mathrm{~mL}$ dry THF was stirred for 30 seconds and filtered through glass wool into a solution of $5.0 \mathrm{mmol}$ (5.0 equiv) alcohol 4 in $0.8 \mathrm{~mL}$ dry THF $(c=1 \mathrm{M})$. Subsequently, $1.0 \mathrm{mmol}$ (1.0 equiv) methyl ester $\mathbf{1}$, and $0.5 \mathrm{~g}$ activated molecular sieve ( $4 \AA$ ) were added and the reaction was continued at ambient temperature until TLC indicated no further change, concentrated under reduced pressure, and the residue purified by flash chromatography $(n$-hexane/ethyl acetate $=10: 1)$.

\section{General procedure B for the transesterification reaction of vinyl esters with NHOs}

A suspension of $0.05 \mathrm{mmol}(5 \mathrm{~mol} \%)$ NHO azolium salt pre-A and $0.05 \mathrm{mmol}(5 \mathrm{~mol} \%)$ potassium tert-butoxide in $0.2 \mathrm{~mL}$ dry THF was stirred for 30 seconds and filtered through glass wool into a solution of $5.0 \mathrm{mmol}$ (5.0 equiv) alcohol 4 in $0.8 \mathrm{~mL}$ dry THF $(c=1 \mathrm{M})$. Subsequently, $1.0 \mathrm{mmol}$ (1.0 equiv) vinyl ester $1 \mathbf{c}$ were added and the reaction was continued at ambient temperature until TLC indicated no further change, concentrated under reduced pressure, and the residue purified by flash chromatography ( $n$-hexane/ethyl acetate $=10: 1$ ).

\section{General procedure $\mathrm{C}$ for the transesterification reaction with in-situ NHO formation}

A suspension of $0.06 \mathrm{mmol}(6 \mathrm{~mol} \%)$ NHO azolium salt pre-A and $0.05 \mathrm{mmol}(5 \mathrm{~mol} \%)$ potassium tert-butoxide in $1.0 \mathrm{~mL}$ dry $\operatorname{THF}(c=1 \mathrm{M})$ was stirred for 30 seconds. Subsequently, $5.0 \mathrm{mmol}$ (5.0 equiv) alcohol 4, $1.0 \mathrm{mmol}$ (1.0 equiv) methyl ester 1 , and $0.5 \mathrm{~g}$ molecular sieve $(4 \AA)$ were added. Then the reaction was continued at ambient temperature until TLC indicated no further change, concentrated under reduced pressure, and the residue purified by flash chromatography $(n$-hexane/ethyl acetate $=10: 1)$. 


\section{Substrate Scope}

Table S4. Substrate scope of the NHO-catalyzed transesterification reaction. ${ }^{[\mathrm{a}]}$

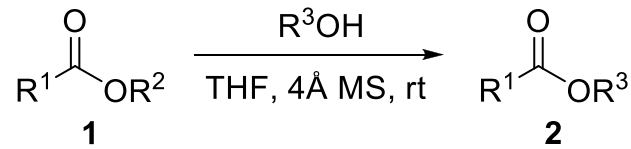<smiles>C=C1N(C)C(C)=C(C)N1C</smiles>

A<smiles></smiles>

C $\left(6 \mathrm{~mol} \%\right.$ pre-A $\left.+5 \mathrm{~mol} \% \mathrm{KO}^{t} \mathrm{Bu}\right)$

\begin{tabular}{|c|c|c|c|c|c|}
\hline 2 & $\mathrm{R}^{1}$ & $\mathrm{R}^{2}$ & $\mathrm{R}^{3}$ & $t[\mathrm{~h}]$ & yield $[\%]^{[\mathrm{b}]}$ \\
\hline $\mathbf{a}$ & $\mathrm{Ph}$ & $\mathrm{Me}$ & $\mathrm{Et}$ & 2 & $80(84)$ \\
\hline b & $\mathrm{Ph}$ & $\mathrm{Me}$ & ${ }^{n} \mathrm{Bu}$ & 3 & $85(86)$ \\
\hline c & $\mathrm{Ph}$ & $\mathrm{Me}$ & ${ }^{n}$ Oct & 3 & 86 \\
\hline d & $\mathrm{Ph}$ & $\mathrm{Me}$ & $\mathrm{Bn}$ & 1.5 & $90(91)$ \\
\hline $\mathbf{e}$ & $(E)-\mathrm{Ph}-\mathrm{CH}=\mathrm{CH}$ & $\mathrm{Me}$ & Et & 2 & 88 \\
\hline f & $(E)-\mathrm{Ph}-\mathrm{CH}=\mathrm{CH}$ & $\mathrm{Me}$ & $\mathrm{Bn}$ & 2 & $79(84)$ \\
\hline g & pyridin-2-yl & $\mathrm{Me}$ & Et & 2 & 82 \\
\hline h & $4-\mathrm{NO}_{2} \mathrm{C}_{6} \mathrm{H}_{4}$ & $\mathrm{Me}$ & Et & 2 & $86(85)$ \\
\hline $\mathbf{i}$ & $4-\mathrm{MeOC}_{6} \mathrm{H}_{4}$ & $\mathrm{Me}$ & ${ }^{n} \mathrm{Bu}$ & 3 & 81 \\
\hline $\mathbf{j}$ & $4-\mathrm{MeOC}_{6} \mathrm{H}_{4}$ & $\mathrm{Me}$ & ${ }^{i} \operatorname{Pr}$ & 12 & 29 \\
\hline $\mathbf{k}$ & $\mathrm{C}_{9} \mathrm{H}_{19}$ & $\mathrm{Me}$ & $\mathrm{Et}$ & 6 & $69(76)$ \\
\hline 1 & $\mathrm{Br}-\mathrm{C}_{5} \mathrm{H}_{10}$ & $\mathrm{Me}$ & $\mathrm{Ph}-\left(\mathrm{CH}_{2}\right)_{2}$ & 3 & 74 \\
\hline $\mathbf{m}$ & $\mathrm{C}_{11} \mathrm{H}_{21}$ & $\mathrm{Me}$ & oct-2-yl & 12 & 24 \\
\hline $\mathbf{n}$ & $\mathrm{C}_{11} \mathrm{H}_{21}$ & $\mathrm{Me}$ & $\mathrm{Cy}$ & 12 & 27 \\
\hline $\mathbf{o}$ & $\mathrm{Me}$ & Et & $\mathrm{Ph}-\left(\mathrm{CH}_{2}\right)_{2}$ & 4 & $89(88)$ \\
\hline $\mathbf{p}$ & 4-TBSOC ${ }_{6} \mathrm{H}_{4}$ & $\mathrm{Me}$ & ${ }^{n} \operatorname{Pr}$ & 4 & 73 \\
\hline $\mathbf{q}$ & $4-\mathrm{TBSOC}_{6} \mathrm{H}_{4}$ & $\mathrm{Me}$ & ${ }^{n} \mathrm{Bu}$ & 4 & 71 \\
\hline $\mathbf{r}$ & 4-THPOC ${ }_{6} \mathrm{H}_{4}$ & $\mathrm{Me}$ & ${ }^{n} \operatorname{Pr}$ & 4 & 66 \\
\hline
\end{tabular}

[a] The reactions were carried out with $1.0 \mathrm{mmol}$ ester 1 and $5.0 \mathrm{mmol}$ alcohol 4 in the presence of $5 \mathrm{~mol} \%$ of catalyst $\mathbf{A}$ in $1 \mathrm{~mL}$ THF $(c=1 \mathrm{M})$ at ambient temperature until the TLC indicated no further change in the reaction mixture. [b] Yield of the isolated product. Yields of the reaction with catalyst $\mathbf{C}$ is given in parentheses. [c] $0.5 \mathrm{~g} 4 \AA$ MS was also added. 


\section{Special Substrates}

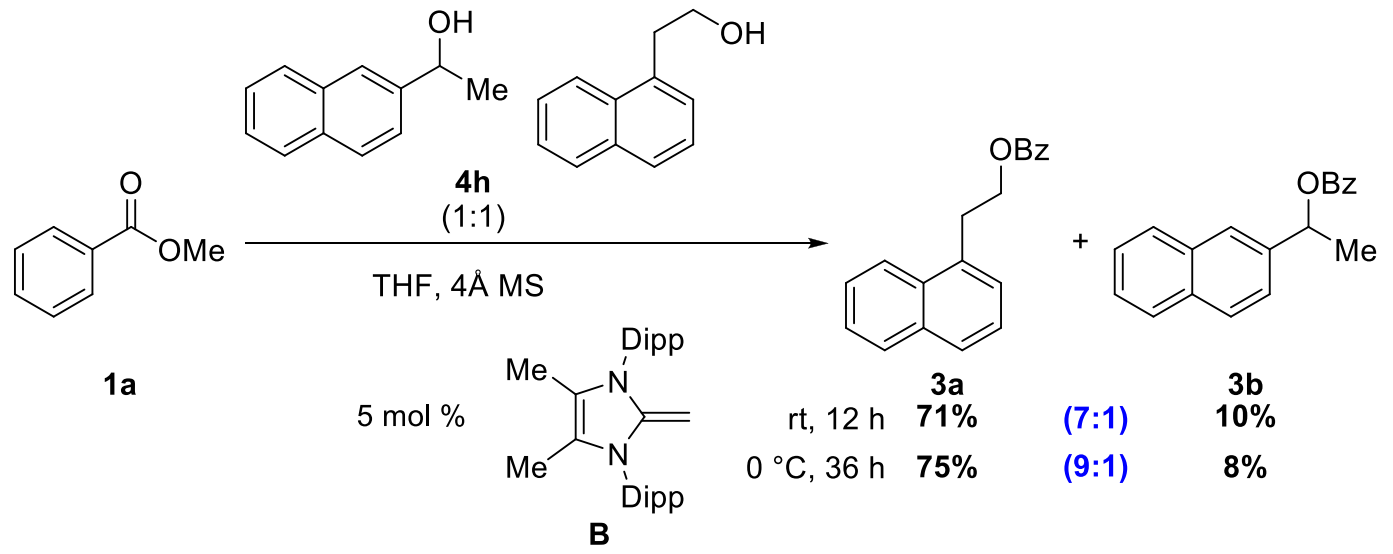

Scheme S1 Selective transesterification reaction with primary alcohols in the presence of secondary alcohols.

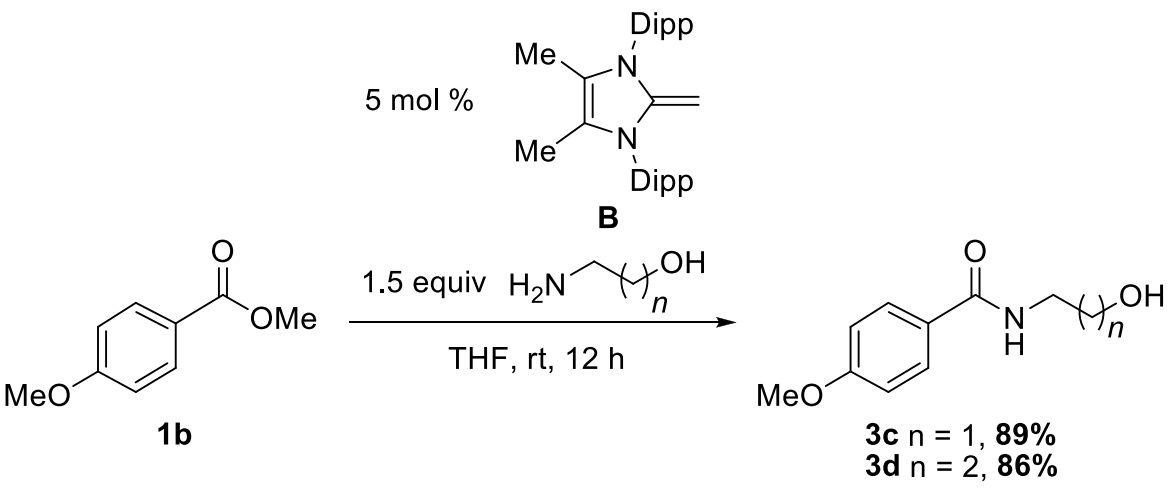

Scheme S2 NHO-catalyzed amidation reaction with aminoalcohols.

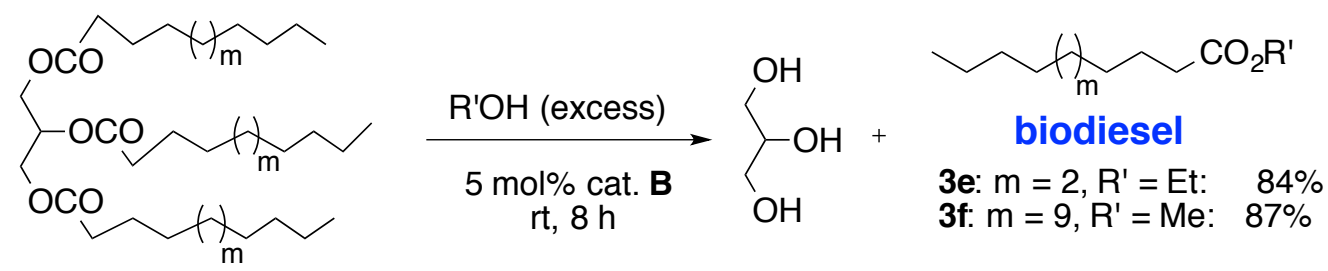

Scheme S3 NHO-catalyzed biodiesel production.<smiles>COC(=O)c1ccc(C(=O)OCCO)cc1</smiles>

Scheme S4 Synthesis of BHET (3g) via the NHO-catalyzed transesterification reaction with ethylene glycol. 
Ethyl benzoate $^{3}$ (Table S4, 2a): Prepared according to the general procedure A from methyl benzoate and ethanol to yield the title compound as colorless oil $(120 \mathrm{mg}, 0.80 \mathrm{mmol}, 80 \%$ yield).<smiles>CCOC(=O)c1ccccc1</smiles>

${ }^{1}$ H NMR (300 MHz, CDCl$) \delta 8.05(\mathrm{~d}, J=7.9 \mathrm{~Hz}, 2 \mathrm{H}), 7.49$ - $7.61(\mathrm{~m}, 1 \mathrm{H}), 7.37-7.49$ (m, 2H), 4.38 (q, $J=7.1 \mathrm{~Hz}, 2 \mathrm{H}), 1.39(\mathrm{t}, J=7.1 \mathrm{~Hz}, 3 \mathrm{H}) \mathrm{ppm} ;{ }^{13} \mathbf{C}$ NMR (75 MHz, CDCl $) \delta$ $166.8,132.9,130.6,129.6,128.4,61.1,14.4 \mathrm{ppm}$.

n-Butyl benzoate ${ }^{4}$ (Table S4, 2b): Prepared according to the general procedure A from methyl benzoate and $n$-butanol to yield the title compound as colorless oil (152 mg, 0.85 mmol, $85 \%$ yield).<smiles>CCCCOC(=O)c1ccccc1</smiles>

${ }^{1}$ H NMR (300 MHz, CDCl$\left._{3}\right) \delta 8.00-8.10(\mathrm{~m}, 2 \mathrm{H}), 7.51-7.60(\mathrm{~m}, 1 \mathrm{H}), 7.39-7.49(\mathrm{~m}, 2 \mathrm{H})$, $4.33(\mathrm{t}, J=6.6 \mathrm{~Hz}, 2 \mathrm{H}), 1.69-1.82(\mathrm{~m}, 2 \mathrm{H}), 1.40-1.57(\mathrm{~m}, 2 \mathrm{H}), 0.98(\mathrm{t}, J=7.4 \mathrm{~Hz}, 3 \mathrm{H})$ ppm; ${ }^{13} \mathbf{C}$ NMR (75 MHz, $\left.\mathbf{C D C l}_{3}\right) \delta 166.8,132.9,130.7,129.7,128.4,65.0,30.9,19.4,13.9$ ppm.

n-Octyl benzoate ${ }^{5}$ (Table S4, 2c): Prepared according to the general procedure A from methyl benzoate and $n$-octanol to yield the title compound as colorless oil (202 $\mathrm{mg}, 0.86$ mmol, $86 \%$ yield).<smiles>O=C(O[Ga])c1ccccc1</smiles>

${ }^{1}$ H NMR (400 MHz, CDCl $) \delta 8.02-8.08(\mathrm{~m}, 2 \mathrm{H}), 7.51-7.58(\mathrm{~m}, 1 \mathrm{H}), 7.39-7.48(\mathrm{~m}, 2 \mathrm{H})$, $4.32(\mathrm{t}, J=6.7 \mathrm{~Hz}, 2 \mathrm{H}), 1.77$ (quin, $J=7.1 \mathrm{~Hz}, 2 \mathrm{H}), 1.21-1.50(\mathrm{~m}, 10 \mathrm{H}), 0.84-0.93(\mathrm{~m}, 3 \mathrm{H})$

\footnotetext{
${ }^{3}$ J. R. Hwu, C.-Y. Hsu, M. L. Jain, Tetrahedron Lett. 2004, 45, 5151-5154.

${ }^{4}$ J. J. Hans, R. W. Driver, S. D. Burke, J. Org. Chem. 2000, 65, 2114-2121.

${ }^{5}$ T. V Nguyen, D. J. M. Lyons, Chem. Commun. 2015, 51, 3131-3134.
} 
ppm; ${ }^{13}$ C NMR (100 MHz, $\left.\mathbf{C D C l}_{3}\right) \delta 166.8,132.9,130.7,129.7,128.4,65.3,31.9,29.4$, $29.3,28.9,26.2,22.8,14.21 \mathrm{ppm}$.

Benzyl benzoate ${ }^{6}$ (Table S4, 2d): Prepared according to the general procedure A from methyl benzoate and benzyl alcohol to yield the title compound as colorless oil (191 mg, 0.90 mmol, 90\% yield).<smiles>O=C(OCc1ccccc1)c1ccccc1</smiles>

${ }^{1}$ H NMR (300 MHz, CDCl $) \delta 8.00$ - $8.19(\mathrm{~m}, 2 \mathrm{H}), 7.30$ - $7.63(\mathrm{~m}, 8 \mathrm{H}), 5.38$ (s, 2H) ppm; ${ }^{13}$ C NMR (75 MHz, $\left.\mathbf{C D C l}_{3}\right) \delta 166.6,136.2,133.2,130.3,129.8,128.7,128.5,128.4,128.3$, $66.8 \mathrm{ppm}$.

Ethyl cinnamate ${ }^{7}$ (Table S4, 2e): Prepared according to the general procedure A from methyl cinnamate and ethanol to yield the title compound as colorless oil (155 mg, 0.88 mmol, $88 \%$ yield).<smiles>CCOC(=O)/C=C/c1ccccc1</smiles>

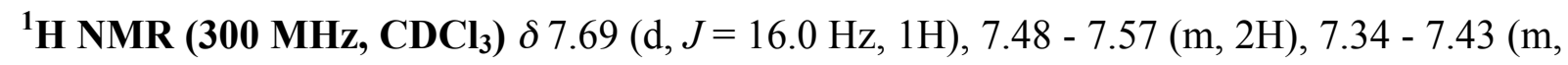
$3 \mathrm{H}), 6.44(\mathrm{~d}, J=16.0 \mathrm{~Hz}, 1 \mathrm{H}), 4.27(\mathrm{q}, J=7.1 \mathrm{~Hz}, 2 \mathrm{H}), 1.34(\mathrm{t}, J=7.1 \mathrm{~Hz}, 3 \mathrm{H}) \mathrm{ppm} ;{ }^{13} \mathbf{C}$ NMR (75 MHz, $\left.\mathbf{C D C l}_{3}\right) \delta 167.1,144.7,134.6,130.3,129.0,128.2,118.4,60.6,14.5$ ppm.

Benzyl cinnamate ${ }^{7}$ (Table S4, 2f): Prepared according to the general procedure A from methyl cinnamate and benzyl alcohol to yield the title compound as colorless solid (188 mg, $0.79 \mathrm{mmol}, 79 \%$ yield).<smiles>O=C(/C=C/c1ccccc1)OCc1ccccc1</smiles>

\footnotetext{
${ }^{6}$ R. Doi, K. Kikushima, M. Ohashi, S. Ogoshi, J. Am. Chem. Soc. 2015, 137, 3276-3282.

${ }^{7}$ B. Zhang, P. Feng, Y. Cui, N. Jiao, Chem. Commun. 2012, 48, 7280-7282.
} 
${ }^{1}$ H NMR (300 MHz, CDCl $) \delta 7.75(\mathrm{~d}, J=16.0 \mathrm{~Hz}, 1 \mathrm{H}), 7.48-7.61(\mathrm{~m}, 2 \mathrm{H}), 7.29-7.48(\mathrm{~m}$, $8 \mathrm{H}), 6.50(\mathrm{~d}, J=16.0 \mathrm{~Hz}, 1 \mathrm{H}), 5.27$ (s, 2H) ppm; ${ }^{13} \mathbf{C}$ NMR (75 MHz, $\left.\mathbf{C D C l}_{3}\right) \delta 166.9$, $145.3,136.2$, 134.5, 130.5, 129.0, 128.7, 128.4, 128.4, 128.2, 118.0, 66.5 ppm.

Ethyl picolinate $^{\mathbf{8}}$ (Table S4, 2g): Prepared according to the general procedure A from methyl picolinate and ethanol to yield the title compound as yellow solid (124 mg, $0.82 \mathrm{mmol}, 82 \%$ yield).<smiles>CCOC(=O)c1ccccn1</smiles>

${ }^{1}$ H NMR (400 MHz, CDCl $)_{3} \delta 8.66\left(\mathrm{ddd}, J_{1}=4.7 \mathrm{~Hz}, J_{2}=1.8 \mathrm{~Hz}, J_{3}=1.0 \mathrm{~Hz}, 1 \mathrm{H}\right), 8.04(\mathrm{dt}$, $\left.J_{1}=7.8 \mathrm{~Hz}, J_{2}=1.0 \mathrm{~Hz}, 1 \mathrm{H}\right), 7.75\left(\mathrm{td}, J_{1}=7.8 \mathrm{~Hz}, J_{2}=1.8 \mathrm{~Hz}, 1 \mathrm{H}\right), 7.38$ (ddd, $J_{1}=7.8 \mathrm{~Hz}$, $\left.J_{2}=4.7 \mathrm{~Hz}, J_{3}=1.0 \mathrm{~Hz}, 1 \mathrm{H}\right), 4.39(\mathrm{q}, J=7.1 \mathrm{~Hz}, 2 \mathrm{H}), 1.35(\mathrm{t}, J=7.1 \mathrm{~Hz}, 3 \mathrm{H}) \mathrm{ppm} ;{ }^{13} \mathbf{C}$ NMR (100 MHz, $\left.\mathbf{C D C l}_{3}\right) \delta 165.1,149.8,148.2,136.9,126.8,125.0,61.9,14.3$ ppm.

Ethyl 4-nitrobenzoate9 (Table S4, 2h): Prepared according to the general procedure A from methyl 4-nitrobenzoate and ethanol to yield the title compound as off-white solid (168 mg, $0.86 \mathrm{mmol}, 86 \%$ yield).<smiles>CCOC(=O)c1ccc([N+](=O)[O-])cc1</smiles>

${ }^{1}$ H NMR (400 MHz, $\left.\mathbf{C D C l}_{3}\right) \delta 8.25-8.30(\mathrm{~m}, 2 \mathrm{H}), 8.18-8.24(\mathrm{~m}, 2 \mathrm{H}), 4.43(\mathrm{q}, J=7.1 \mathrm{~Hz}$, 2H), $1.42(\mathrm{t}, J=7.1 \mathrm{~Hz}, 3 \mathrm{H}) \mathrm{ppm} ;{ }^{13} \mathbf{C}$ NMR (100 MHz, $\left.\mathbf{C D C l}_{3}\right) \delta$ 164.8, 150.6, 136.0, $130.8,123.6,62.1,14.4 \mathrm{ppm}$.

n-Butyl 4-methoxybenzoate ${ }^{10}$ (Table S4, 2i): Prepared according to the general procedure A from methyl 4-methoxybenzoate and $n$-butanol to yield the title compound as colorless oil (169 mg, $0.81 \mathrm{mmol}, 81 \%$ yield).

${ }^{8}$ B. Riflade, D. Lachkar, J. Oble, J. Li, S. Thorimbert, B. Hasenknopf, E. Lacôte, Org. Lett. 2014, 16, 3860-3863.

${ }^{9}$ W. Han, F. Jin, Q. Zhou, Synthesis 2015, 47, 1861-1868.

${ }^{10}$ A. M. Whittaker, V. M. Dong, Angew. Chemie Int. Ed. 2015, 54, 1312-1315. 
<smiles>CCCCOC(=O)c1ccc(OC)cc1</smiles>

${ }^{1}$ H NMR (300 MHz, CDCl$)_{3} \delta 7.99(\mathrm{~d}, J=8.8 \mathrm{~Hz}, 2 \mathrm{H}), 6.91(\mathrm{~d}, J=8.8 \mathrm{~Hz}, 2 \mathrm{H}), 4.29$ (t, $\mathrm{J}=6.6 \mathrm{~Hz}, 2 \mathrm{H}), 3.85(\mathrm{~s}, 3 \mathrm{H}), 1.66-1.81(\mathrm{~m}, 2 \mathrm{H}), 1.47(\mathrm{sxt}, \mathrm{J}=7.4 \mathrm{~Hz}, 2 \mathrm{H}), 0.97$ (t, J = 7.4 $\mathrm{Hz}, 3 \mathrm{H}) \mathrm{ppm} ;{ }^{13} \mathbf{C}$ NMR (75 MHz, $\left.\mathbf{C D C l}_{3}\right) \delta$ 166.6, 163.4, 131.7, 123.1, 113.7, 64.7, 55.5, 31.0, 19.4, $13.9 \mathrm{ppm}$.

iso-Propyl 4-methoxybenzoate ${ }^{11}$ (Table S4, 2j): Prepared according to the general procedure A from methyl 4-methoxybenzoate and iso-propanol to yield the title compound as colorless oil (56 mg, $0.29 \mathrm{mmol}, 29 \%$ yield).<smiles>CCCOC(=O)c1ccc(OC)cc1</smiles>

${ }^{1}$ H NMR (300 MHz, CDCl$)_{3} \delta 7.99(\mathrm{~d}, J=8.6 \mathrm{~Hz}, 2 \mathrm{H}), 6.90(\mathrm{~d}, J=8.6 \mathrm{~Hz}, 2 \mathrm{H}), 5.22$ (spt, $J=6.2 \mathrm{~Hz}, 1 \mathrm{H}), 3.85(\mathrm{~s}, 3 \mathrm{H}), 1.35(\mathrm{~d}, J=6.2 \mathrm{~Hz}, 6 \mathrm{H}) \mathrm{ppm} ;{ }^{13} \mathbf{C} \mathbf{N M R}\left(\mathbf{7 5} \mathbf{M H z}, \mathbf{C D C l}_{3}\right) \delta$ $166.0,163.3,131.6,123.5,113.6,68.0,55.5,22.1 \mathrm{ppm}$.

Ethyl decanoate $^{\mathbf{1 2}}$ (Table S4, 2k): Prepared according to the general procedure A from methyl hexanoate and ethanol to yield the title compound as colorless oil (138 mg, 0.69 mmol, 69\% yield).<smiles>CCCCCCCCCC(=O)OCC</smiles>

${ }^{1}$ H NMR (400 MHz, $\left.\mathbf{C D C l}_{3}\right) \delta 4.11(\mathrm{q}, J=7.2 \mathrm{~Hz}, 2 \mathrm{H}), 2.27(\mathrm{t}, J=7.6 \mathrm{~Hz}, 2 \mathrm{H}), 1.55-1.65$ $(\mathrm{m}, 2 \mathrm{H}), 1.17$ - $1.36(\mathrm{~m}, 15 \mathrm{H}), 0.82$ - $0.91(\mathrm{~m}, 3 \mathrm{H}) \mathrm{ppm} ;{ }^{13} \mathbf{C}$ NMR (100 MHz, CDCl $) \delta$ 174.0, 60.3, 34.5, 32.0, 29.5, 29.4 (two coincident resonances), 29.3, 25.1, 22.8, 14.4, 14.2 ppm.

${ }^{11}$ W. Guo, L.-Q. Lu, Y. Wang, Y.-N. Wang, J.-R. Chen, W.-J. Xiao, Angew. Chemie Int. Ed. 2015, 54, 2265-2269.

${ }^{12}$ L. Guo, X. Ma, H. Fang, X. Jia, Z. Huang, Angew. Chem. Int. Ed. 2015, 54, 4023-4027. 
Phenethyl 6-bromohexanoate (Table S4, 21): Prepared according to the general procedure A from methyl 6-bromohexanoate and 2-phenylethanol to yield the title compound as colorless oil (221 mg, $0.74 \mathrm{mmol}, 74 \%$ yield).

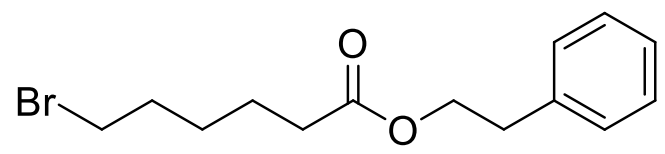

${ }^{1}$ H NMR (300 MHz, CDCl$\left.)_{3}\right) \delta 7.15-7.34(\mathrm{~m}, 5 \mathrm{H}), 4.28(\mathrm{t}, J=7.0 \mathrm{~Hz}, 2 \mathrm{H}), 3.35$ (t, $J=6.8$ $\mathrm{Hz}, 2 \mathrm{H}), 2.92(\mathrm{t}, J=7.0 \mathrm{~Hz}, 2 \mathrm{H}), 2.28$ (t, $J=7.3 \mathrm{~Hz}, 2 \mathrm{H}), 1.75-1.89$ (m, 2H), 1.52 - 1.67 $(\mathrm{m}, 2 \mathrm{H}), 1.33$ - 1.47 (m, 2H) ppm; ${ }^{13} \mathbf{C}$ NMR (75 MHz, $\left.\mathbf{C D C l}_{3}\right) \delta 173.5,138.0,129.0,128.6$, 126.7, 64.9, 35.2, 34.2, 33.6, 32.5, 27.7, 24.2 ppm; IR: 2981, $1722 \mathrm{~cm}^{-1}$; HRMS (ESI-MS): $m / z$ calcd. for $\mathrm{C}_{14} \mathrm{H}_{19} \mathrm{BrO}_{2}[\mathrm{M}+\mathrm{Na}]^{+}=321.0466$ and 323.0446, found 321.0464 and 323.0440 .

Octan-2-yl dodecanoate $^{13}$ (Table S4, 2m): Prepared according to the general procedure A from methyl hexanoate and octan-2-ol to yield the title compound as colorless oil $(75 \mathrm{mg}$, $0.24 \mathrm{mmol}, 24 \%$ yield).

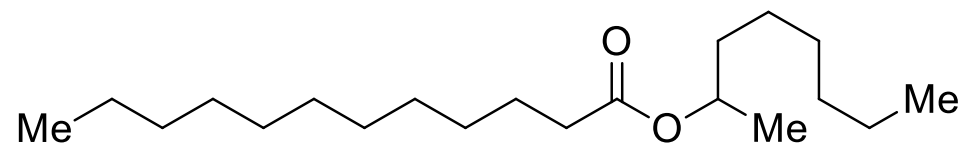

${ }^{1}$ H NMR (400 MHz, CDCl $) \delta 4.83-4.96(\mathrm{~m}, 1 \mathrm{H}), 2.26(\mathrm{t}, J=7.5 \mathrm{~Hz}, 2 \mathrm{H}), 1.42-1.66(\mathrm{~m}$, 4H), 1.23 - 1.31 (m, 24H), 1.19 (d, $J=6.3 \mathrm{~Hz}, 3 \mathrm{H}), 0.82$ - 0.94 (m, 6H) ppm; ${ }^{13} \mathbf{C}$ NMR (100 MHz, $\left.\mathbf{C D C l}_{3}\right) \delta 173.7,70.9,36.1,34.9,32.1,31.9,29.8,29.6,29.5,29.4,29.3,29.3,25.5$, $25.3,22.8,22.7,20.2,14.2,14.2 \mathrm{ppm}$.

Cyclohexyl dodecanoate $^{13}$ (Table S4, 2n): Prepared according to the general procedure A from methyl hexanoate and cyclohexanol to yield the title compound as colorless oil $(75 \mathrm{mg}$, $0.27 \mathrm{mmol}, 27 \%$ yield).

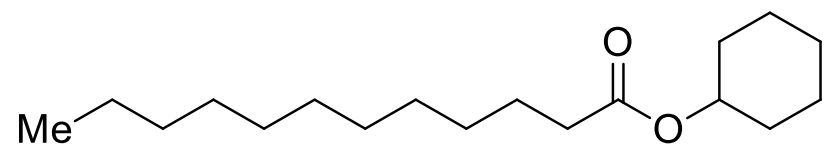

${ }^{1}$ H NMR (400 MHz, CDCl $) \delta 4.71-4.79(\mathrm{~m}, 1 \mathrm{H}), 2.26(\mathrm{t}, J=7.5 \mathrm{~Hz}, 2 \mathrm{H}), 1.78-1.88(\mathrm{~m}$, 2H), 1.66 - $1.77(\mathrm{~m}, 2 \mathrm{H}), 1.53$ - $1.63(\mathrm{~m}, 2 \mathrm{H}), 1.23$ - $1.43(\mathrm{~m}, 22 \mathrm{H}), 0.82$ - 0.92 (m, 3H) ppm; ${ }^{13} \mathbf{C}$ NMR (100 MHz, $\left.\mathbf{C D C l}_{3}\right) \delta 173.5,72.4,34.9,32.1,31.8,29.7,29.6,29.5,29.4,29.3$, 25.6, 25.3, 23.9, 22.8, $14.2 \mathrm{ppm}$.

${ }^{13}$ T. V. Nguyen, D. J. M. Lyons, Chem. Commun. 2015, 51, 3131-3134. 
Phenethyl acetate ${ }^{14}$ (Table S4, 2o): Prepared according to the general procedure A from ethyl acetate and 2-phenylethanol to yield the title compound as yellow oil (146 mg, 0.89 mmol, $89 \%$ yield).<smiles>CC(=O)OCCc1ccccc1</smiles>

${ }^{1}$ H NMR (300 MHz, CDCl $) \delta 7.18-7.38(\mathrm{~m}, 5 \mathrm{H}), 4.30(\mathrm{t}, J=7.1 \mathrm{~Hz}, 2 \mathrm{H}), 2.95(\mathrm{t}$, $J=7.1 \mathrm{~Hz}, 2 \mathrm{H}), 2.04$ (s, 3H) ppm; ${ }^{13} \mathbf{C} \mathbf{N M R}\left(\mathbf{7 5} \mathbf{M H z}, \mathbf{C D C l}_{3}\right) \delta 171.1,137.9,128.9,128.6$, $126.6,65.0,35.1,21.0 \mathrm{ppm}$.

n-Propyl 4-(tert-butyldimethylsilyloxy)benzoate ${ }^{15}$ (Table S4, 2p): Prepared according to the general procedure A from methyl 4-(tert-butyldimethylsilyloxy)benzoate and propanol to yield the title compound as a colorless solid ( $214 \mathrm{mg}, 0.73 \mathrm{mmol}, 73 \%$ yield).<smiles>CCCOC(=O)c1ccc(OS(=O)(=[Te])c2ccccc2)cc1</smiles>

${ }^{1}$ H NMR (300 MHz, CDCl $) \delta 7.89-8.00(\mathrm{~m}, 2 \mathrm{H}), 6.79-6.91(\mathrm{~m}, 2 \mathrm{H}), 4.24(\mathrm{t}, J=6.7 \mathrm{~Hz}$, 2H), 1.77 (sxt, $J=7.1 \mathrm{~Hz}, 2 \mathrm{H}), 0.93-1.08(\mathrm{~m}, 12 \mathrm{H}), 0.17-0.27(\mathrm{~m}, 6 \mathrm{H}) \mathrm{ppm} ;{ }^{13} \mathbf{C}$ NMR (75 MHz, $\left.\mathbf{C D C l}_{3}\right) \delta 166.6,160.1,131.6,123.7,119.9,66.4,25.7,22.3,18.4,10.7,-4.3$ ppm.

n-Butyl 4-(tert-butyldimethylsilyloxy)benzoate ${ }^{15}$ (Table S4, 2q): Prepared according to the general procedure A from methyl 4-(tert-butyldimethylsilyloxy)benzoate and butanol to yield the title compound as a colorless solid ( $218 \mathrm{mg}, 0.71 \mathrm{mmol}, 71 \%$ yield).<smiles>CCCCOc1ccc(C(=O)Oc2ccccc2)cc1</smiles>

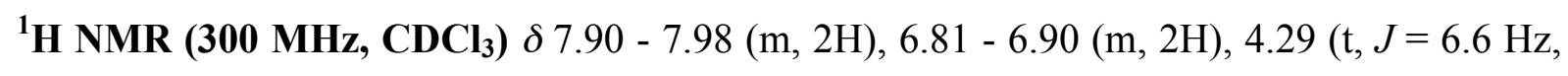
2H), $1.66-1.80(\mathrm{~m}, 2 \mathrm{H}), 1.39-1.55(\mathrm{~m}, 2 \mathrm{H}), 0.92-1.03(\mathrm{~m}, 12 \mathrm{H}), 0.22(\mathrm{~s}, 6 \mathrm{H}) \mathrm{ppm} ;{ }^{13} \mathbf{C}$ NMR (75 MHz, $\left.\mathbf{C D C l}_{3}\right) \delta$ 166.6, 160.1, 131.6, 123.7, 119.9, 64.7, 31.0, 25.7, 19.4, 18.4, $13.9,-4.3 \mathrm{ppm}$.

${ }^{14}$ T. Hashimoto, D. Hirose, T. Taniguchi, Adv. Synth. Catal. 2015, 357, 3346-3352.
${ }^{15}$ J.-H. Liu, C.-D. Hsieh, H.-Y. Wang, J. Polym. Sci. A Polym. Chem. 2004, 42, 1075-1092. 
n-Propyl 4-((tetrahydro-2H-pyran-2-yl)oxy)benzoate ${ }^{15}$ (Table S4, 2r): Prepared according to the general procedure A from methyl 4-((tetrahydro-2H-pyran-2-yl)oxy)benzoate and propanol to yield the title compound as a colorless solid (174 mg, $0.66 \mathrm{mmol}, 66 \%$ yield).<smiles>O=C(O[Ga])c1ccc(OP)cc1</smiles>

${ }^{1}$ H NMR (300 MHz, CDCl $) \delta 7.93-8.06(\mathrm{~m}, 2 \mathrm{H}), 7.01-7.12(\mathrm{~m}, 2 \mathrm{H}), 5.50(\mathrm{t}, J=3.1 \mathrm{~Hz}$, $1 \mathrm{H}), 4.25(\mathrm{t}, J=6.6 \mathrm{~Hz}, 2 \mathrm{H}), 3.76-3.95(\mathrm{~m}, 1 \mathrm{H}), 3.54-3.68(\mathrm{~m}, \mathrm{H}), 1.60-2.11(\mathrm{~m}, 8 \mathrm{H})$, $1.02(\mathrm{t}, J=7.4 \mathrm{~Hz}, 3 \mathrm{H}) \mathrm{ppm} ;{ }^{13} \mathbf{C}$ NMR (75 MHz, $\left.\mathbf{C D C l}_{3}\right) \delta 166.6,160.9,131.5,123.8$, $116.0,96.2,66.4,62.2,30.3,25.2,22.3,18.7,10.7$ ppm.

2-(Naphthalen-1-yl)ethyl benzoate ${ }^{16}$ (Scheme S1, 3a): Prepared according to the general procedure A with cat. B from methyl benzoate and 1:1-mixture of 1-(naphthalen-2-yl)ethanol and 2-(naphthalen-1-yl)ethanol to yield the title compound as colorless solid (196 mg, 0.71 mmol, $71 \%$ yield).<smiles>O=C(OCCc1cccc2ccccc12)c1ccccc1</smiles>

${ }^{1}$ H NMR (300 MHz, CDCl $) \delta 8.20(\mathrm{~d}, J=8.4 \mathrm{~Hz}, 1 \mathrm{H}), 7.99-8.10(\mathrm{~m}, 2 \mathrm{H}), 7.89(\mathrm{~d}$, $J=8.1 \mathrm{~Hz}, 1 \mathrm{H}), 7.74-7.85(\mathrm{~m}, 1 \mathrm{H}), 7.37-7.64(\mathrm{~m}, 7 \mathrm{H}), 4.69(\mathrm{t}, J=7.3 \mathrm{~Hz}, 2 \mathrm{H}), 3.58$ (t, $J=7.3 \mathrm{~Hz}, 2 \mathrm{H}) \mathrm{ppm} ;{ }^{13} \mathbf{C}$ NMR (75 MHz, $\left.\mathbf{C D C l}_{3}\right) \delta 166.8,134.0,133.9,133.1,132.2,130.4$, $129.7,129.0,128.5,127.6,127.2,126.4,125.8,125.7,123.8,65.1,32.5$ ppm.

1-(Naphthalen-1-yl)ethyl benzoate ${ }^{17}$ (Scheme S1, 3b): Prepared according to the general procedure A with cat. B from methyl benzoate and 1:1-mixture of 1- and 2-(naphthalen-1yl)ethanol to yield the title compound as colorless solid (28 mg, $0.10 \mathrm{mmol}, 10 \%$ yield).<smiles>CC(OC(=O)c1ccccc1)c1ccc2ccccc2c1</smiles>

${ }^{16}$ C. Zhang, P. Feng, N. Jiao, J. Am. Chem. Soc. 2013, 135, 15257-15262.

17 S. V. Slungård, T.-A. Krakeli, T. H. K. Thvedt, E. Fuglseth, E. Sundby, B. H. Hoff, Tetrahedron 2011, 67, 5642-5650. 
${ }^{1}$ H NMR (400 MHz, CDCl $) \delta 8.11(\mathrm{~d}, J=8.2 \mathrm{~Hz}, 2 \mathrm{H}), 7.79-7.95$ (m, 4H), 7.39 - 7.65 (m, $6 \mathrm{H}), 6.31(\mathrm{q}, J=6.6 \mathrm{~Hz}, 1 \mathrm{H}), 1.77(\mathrm{~d}, J=6.6 \mathrm{~Hz}, 3 \mathrm{H}) \mathrm{ppm} ;{ }^{13} \mathbf{C} \mathbf{N M R}\left(151 \mathbf{M H z}, \mathbf{C D C l}_{\mathbf{3}}\right) \delta$ $165.98,139.23,133.33,133.17,133.08,130.64,129.80,128.57,128.49,128.19$, 127.80, $126.37,126.20,125.14,124.21,73.20,22.49$ ppm.

N-(2-hydroxyethyl)-4-methoxybenzamide ${ }^{18}$ (Scheme S2, 3c): Prepared according to the general procedure A with cat. B and without molecular sieve from methyl benzoate and 2aminoethanol to yield the title compound as yellow oil (174 mg, $0.89 \mathrm{mmol}, 89 \%$ yield).<smiles>COc1ccc(C(=O)NCCO)cc1</smiles>

${ }^{1}$ H NMR (300 MHz, CDCl $) \delta 7.73(\mathrm{~d}, J=8.8 \mathrm{~Hz}, 2 \mathrm{H}), 6.88(\mathrm{~d}, J=8.8 \mathrm{~Hz}, 2 \mathrm{H}), 6.79$ (br. s., 1H), 3.73 - 3.89 (m, 5H), 3.58 (q, $J=5.0$ Hz, 2H), 2.84 (br. s., 1H) ppm; ${ }^{13}$ C NMR (75 MHz, $\left.\mathbf{C D C l}_{3}\right) \delta 168.4,162.4,129.0,126.4,113.9,62.5,55.5,43.0 \mathrm{ppm}$.

$N$-(3-hydroxypropyl)-4-methoxybenzamide ${ }^{19}$ (Scheme S2, 3d): Prepared according to the general procedure A with cat. B and without molecular sieve from methyl benzoate and 3aminopropanol to yield the title compound as yellow oil (180 mg, $0.86 \mathrm{mmol}, 86 \%$ yield).<smiles>COc1ccc(C(=O)NCCCO)cc1</smiles>

${ }^{1}$ H NMR (300 MHz, CDCl $) \delta 7.72(\mathrm{~d}, J=8.5 \mathrm{~Hz}, 2 \mathrm{H}), 7.10$ (br. s., $\left.1 \mathrm{H}\right), 6.85$ (d, $J=8.5 \mathrm{~Hz}$, 2H), 3.80 (s, 3H), 3.67 (t, $J=5.7 \mathrm{~Hz}, 2 \mathrm{H}), 3.55$ (q, $J=5.7 \mathrm{~Hz}, 2 \mathrm{H}), 3.44$ (br. s., 1H), 1.74 (quin, $J=5.7 \mathrm{~Hz}, 2 \mathrm{H}) \mathrm{ppm} ;{ }^{13} \mathbf{C}$ NMR (75 MHz, $\left.\mathbf{C D C l}_{3}\right) \delta 168.3,162.3,128.9,126.5,113.8$, $59.8,55.5,37.2,32.1 \mathrm{ppm}$.

Ethyl decanoate (Scheme S3, 3e): Prepared according to the general procedure A with cat. B and without molecular sieve from capric triglyceride and 20 equiv of ethanol to yield the title compound as a colorless oil (156 mg, $0.84 \mathrm{mmol}, 84 \%$ yield). This compound was also reported as compound $\mathbf{2 k}$ above.

\footnotetext{
${ }^{18}$ S. Srinivas Kotha, S. Badigenchala, G. Sekar, Adv. Synth. Catal. 2015, 357, 1437-1445.

${ }^{19}$ P. Ramesh, N. W. Fadnavis, Chem. Lett. 2015, 44, 138-140.
} 
Methyl octadecanoate ${ }^{13}$ (Scheme S3, 3f): Prepared according to the general procedure A with cat. B and without molecular sieve from stearic triglyceride and 20 equiv of methanol to yield the title compound as a white solid (259 mg, $0.87 \mathrm{mmol}, 87 \%$ yield).

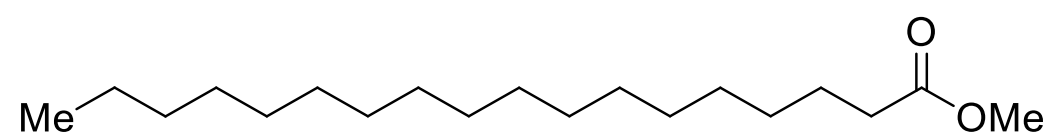

${ }^{1}$ H NMR (300 MHz, CDCl $) \delta 3.66(\mathrm{~s}, 3 \mathrm{H}), 2.30(\mathrm{t}, J=7.5 \mathrm{~Hz}, 2 \mathrm{H}), 1.57-1.70(\mathrm{~m}, 2 \mathrm{H})$, $1.18-1.34(\mathrm{~m}, 28 \mathrm{H}), 0.88(\mathrm{t}, J=6.7 \mathrm{~Hz}, 3 \mathrm{H}) \mathrm{ppm} ;{ }^{13} \mathbf{C}$ NMR (75 MHz, $\left.\mathbf{C D C l}_{3}\right) \delta 174.5$, 51.6, 34.3, 32.1, 29.8, 29.7, 29.6, 29.5, 29.4, 29.3, 25.1, 22.8, 14.3 ppm.

Bis(2-hydroxyethyl) terephthalate ${ }^{20}$ (BHET, Scheme S4, 3g): Prepared according to the general procedure A from methyl terephthalate and ethylene glycol (4.0 equiv) to yield the title compound as colorless solid (236 mg, $0.93 \mathrm{mmol}, 93 \%$ yield). Characterization data corresponded to that of the commercially available BHET.<smiles>O=C(OCCO)c1ccc(C(=O)OCCO)cc1</smiles>

${ }^{1}$ H NMR (300 MHz, d6-DMSO) $\delta 8.08-8.12(\mathrm{~m}, 4 \mathrm{H}), 4.94-4.98$ (m, 2H), $4.31-4.33$ (m, 4H), $3.69-3.74(\mathrm{~m}, 4 \mathrm{H}) \mathrm{ppm}$.

${ }^{20}$ Q. Wang, X. Yao, Y. Geng, Q. Zhou, X. Lu, S. Zhang, Green Chem. 2015, 17, 2473-2479. 


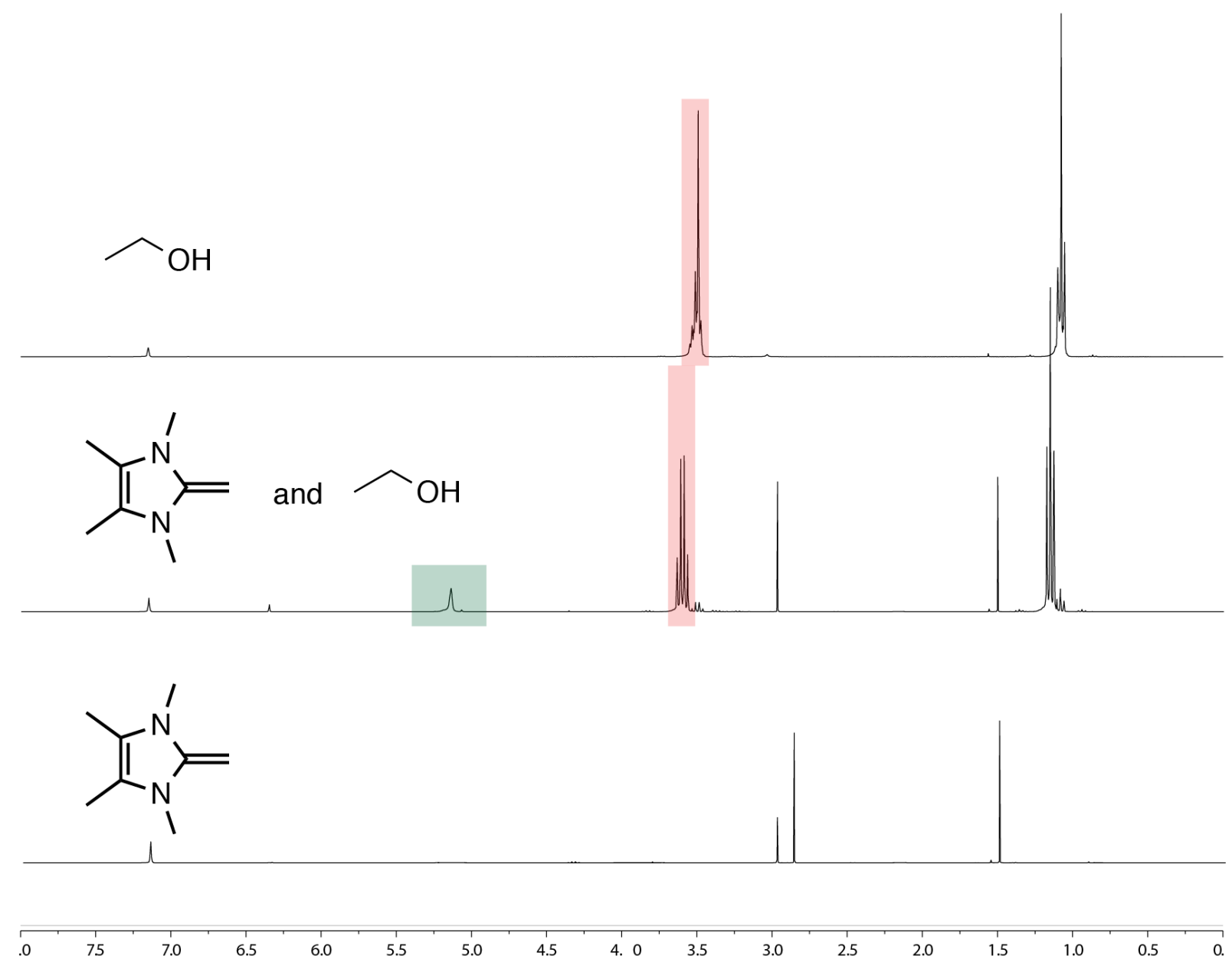

Figure S1. ${ }^{1} \mathrm{H}$ NMR spectra $\left(\mathrm{C}_{6} \mathrm{D}_{6}, 25{ }^{\circ} \mathrm{C}, 300 \mathrm{MHz}\right)$ of (top) EtOH; (middle) NHO A and EtOH mixture; (bottom) NHO A.

When NHO A and EtOH were mixed together, the methylene group ( $\mathrm{HO}-\mathbf{C H}_{\mathbf{2}}-\mathrm{CH}_{3}$, highlighted in pink) of EtOH shifted downfield. A broad signal appreared at $\sim 5.2 \mathrm{ppm}$, most likely representing the hydroxylic proton $\left(\mathrm{HO}-\mathrm{CH}_{2}-\mathrm{CH}_{3}\right.$, highlighted in green) of EtOH. The usually downfield chemical shift was probably the evidence for the deshielded - $\mathrm{OH}$ group that was partially abstracted by the strongly basic NHO A. This complexation study is in good agreement with Naumann's and Dove's studies of NHO A vs. BnOH system. ${ }^{21}$

${ }^{21}$ Naumann, S.; Thomas, A. W.; Dove, A. P. Angew. Chem. Int. Ed. 2015, 54, 9550-9554. 


\section{NHO-catalyzed depolymerization reaction of PET}

PET taken from post-consumer clear bottles from which the polyethylene caps and the polypropylene label were removed was used. The bottles were cut into $\sim 2$ x $6 \mathrm{~mm}$ flakes. PET flakes were washed thoroughly with water then dried under reduced pressure at $50{ }^{\circ} \mathrm{C}$ for 12 hours prior to use.

The PET depolymerization experiments were conducted in a CEM microwave reactor equipped with a digital temperature control system and pressure sensors. PET flakes $(0.5 \mathrm{~g})$ together with $3.0 \mathrm{~mL}$ of ethyleneglycol and catalyst $\mathbf{B}(5 \mathrm{~mol} \%)$ were added into the reaction tube containing a magnetic stirrer bar. The reaction tube was sealed under a nitrogen atmosphere and irradiated using a temperature program (ramping from 25 to $200{ }^{\circ} \mathrm{C}$ then holding at $200{ }^{\circ} \mathrm{C}$ for $60 \mathrm{~min}$ ). The reaction mixture became clear with no sign of PET flakes at this point of the experiment. The reaction mixture was subsequently cooled to room temperature and poured into a conical flask containing $30 \mathrm{~mL}$ hexanes. The resulting white precipitate was collected by filtration and dried under reduced pressure at $50{ }^{\circ} \mathrm{C}$ for 4 hours to yield the BHET product in $94 \%$ yield. Characterization data corresponded to that of the commercially available BHET.

A similar reaction setup under conventional heating conditions with a hotplate stirrer at $200{ }^{\circ} \mathrm{C}$ took $8 \mathrm{~h}$ to achieve $91 \%$ conversion.

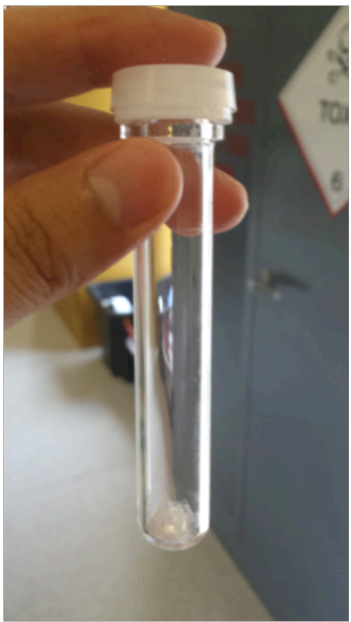

(PET flake)

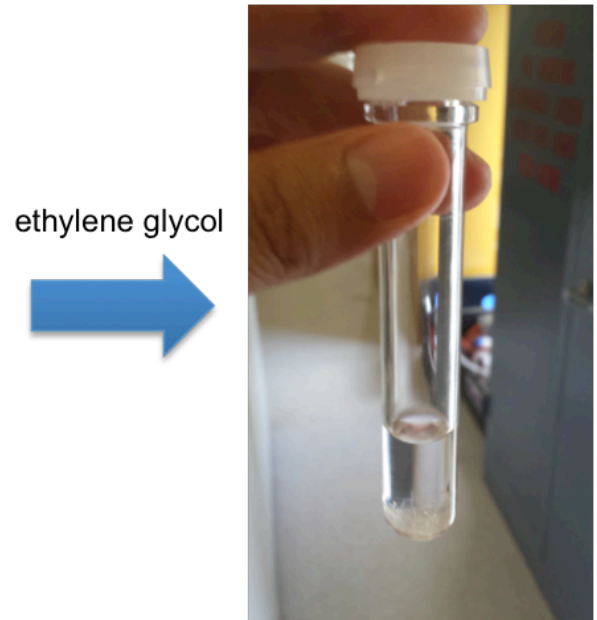

(PET flake in EG)

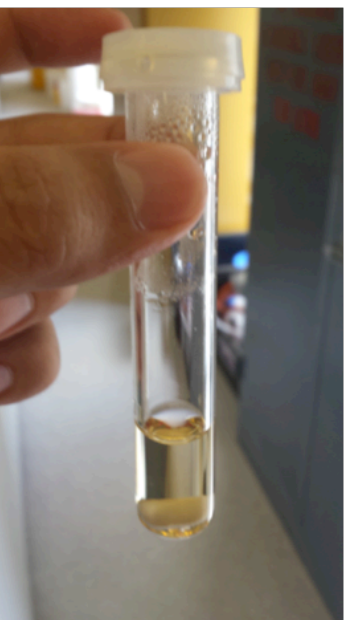

(BHET solution in EG) 


\section{Synthesis of polyesters via an NHO-catalyzed transesterification reaction}

\section{Materials}

Benzyl alcohol (Fluka, puriss p.a.), 3,6-dimethyl-1,4-dioxane-2,5-dione (lactide; Aldrich), anhydrous tetrahydrofuran (THF; Aldrich, $>99 \%$ ) were used without any further purification. $\varepsilon$-caprolactone (Aldrich, 97\%) was purified by vacuum distillation.

\section{Instrumentation}

Size exclusion chromatography (SEC) was performed on a Shimadzu modular system equipped with a Phenomenex $50 \times 7.8 \mathrm{~mm}$ guard column and four Phenogel $300 \times 7.8 \mathrm{~mm}$ linear columns $(500,103,104$, and $105 \AA$ pore size, $5 \mu \mathrm{m}$ particle size). Tetrahydrofuran (THF; HPLC grade) at a flow rate of $1 \mathrm{~mL} \min ^{-1}$ and a constant temperature of $50{ }^{\circ} \mathrm{C}$ was used as the mobile phase with an injection volume of $50 \mu \mathrm{L}$. The system was calibrated with a series of narrow molar mass distribution polystyrene standards with molar masses ranging from $0.58-1820 \mathrm{~kg} \mathrm{~mol}^{-1}$.

\section{Synthesis}

Polymerization of $\varepsilon$-caprolactone by ring-opening polymerization (ROP). $\varepsilon$-caprolactone ( $2.11 \mathrm{~g}, 18.50 \mathrm{mmol}, 100$ equiv) and benzyl alcohol ( $0.02 \mathrm{~g}, 0.19 \mathrm{mmol}, 1$ equiv) were placed in a dry Schlenk flask under nitrogen atmosphere. The catalyst $\mathbf{B}(0.0185 \mathrm{mmol}, 0.1$ equiv) was dissolved in anhydrous toluene $(1 \mathrm{~mL})$, filtered, and added to the reaction mixture, this was then stirred at $110^{\circ} \mathrm{C}$ for 17 hours. After cooling down to room temperature, the viscous solution was diluted with chloroform and then precipitated in a mixture of diethylether- $n$ hexane $(1: 1)$ and the solid was then dried under reduced pressure at $40^{\circ} \mathrm{C}$. The monomer conversion was calculated to be $97 \%$ by ${ }^{1} \mathrm{H}$ NMR yielding 97 repeating units of $\varepsilon$ caprolactone, $\mathrm{Mn}_{\text {theo }}=11.17 \mathrm{~kg} \mathrm{~mol}^{-1}, \mathrm{Mn}_{\text {(SEC THF })}=18.21 \mathrm{~kg} \mathrm{~mol}^{-1}, \bigoplus_{\mathrm{SEC}}=1.71$.

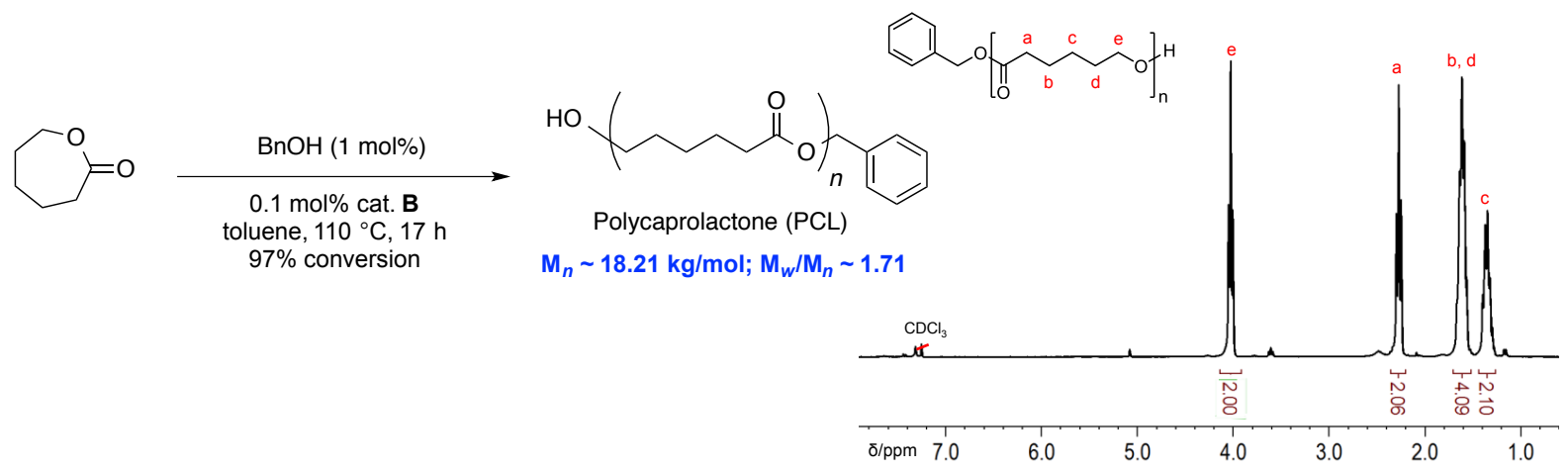


Polymerization of $\boldsymbol{L}, \boldsymbol{D}$-Lactide by ring-opening polymerization (ROP). 3,6-dimethyl-1,4dioxane-2,5-dione (1.33 g, $9.25 \mathrm{mmol}, 50$ equiv) and benzyl alcohol (0.02 g, $0.19 \mathrm{mmol}, 1$ equiv) were weight in a dry Schlenk flask. Catalyst $\mathbf{B}(0.0185 \mathrm{mmol}, 0.1$ equiv), dissolved in anhydrous toluene $(1 \mathrm{~mL})$ and filtered, was added to the mixture under nitrogen atmosphere. The Schlenk flask was then placed in a $110^{\circ} \mathrm{C}$ pre heated oil bath and stirred for 25 hours. After cooling to room temperature, the polymer was precipitated in cold methanol and then dried under reduced pressure at $40^{\circ} \mathrm{C}$ to obtain a colourless solid. The monomer conversion was calculated to be $98 \%$ by ${ }^{1} \mathrm{H}$ NMR yielding 98 repeating units of $L, D$-lactide, $\mathrm{Mn}_{\text {theo }}=$ $7.17 \mathrm{~kg} \mathrm{~mol}^{-1}, \mathrm{Mn}_{\text {(SEC THF })}=9.56 \mathrm{~kg} \mathrm{~mol}^{-1}, \mathrm{Ð}_{\mathrm{SEC}}=1.56$.<smiles>CC1OC(=O)C(C)OC1=O</smiles>
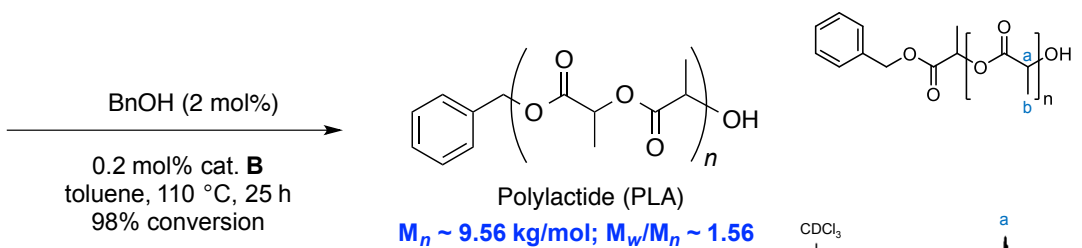
$\mathrm{M}_{n} \sim 9.56 \mathrm{~kg} / \mathrm{mol} ; \mathrm{M}_{w} / \mathrm{M}_{n} \sim 1.56$
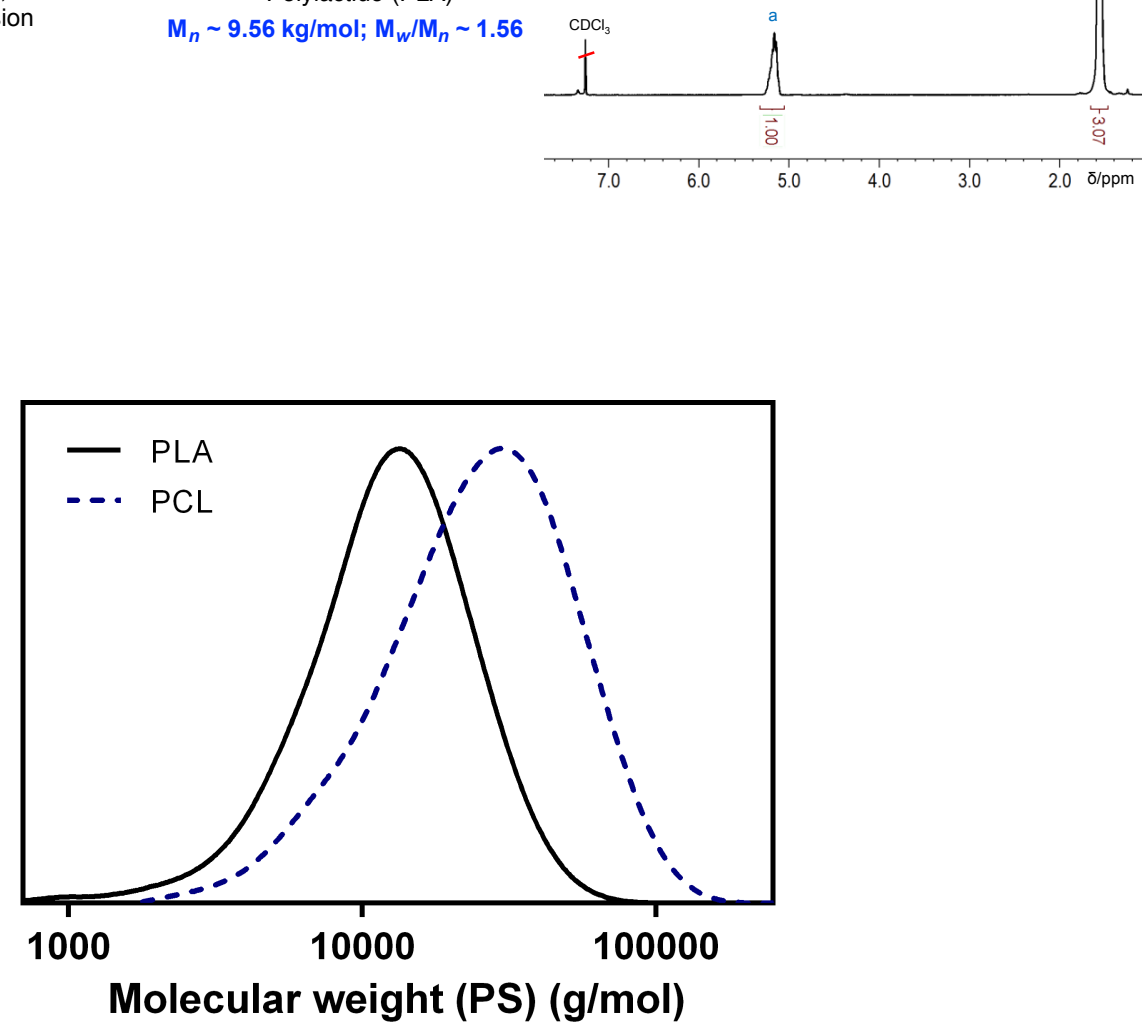

Figure S1 Molecular weight distribution of polyester products. 
Using a volumetric flask $7.6 \mathrm{mg}(49.9 \mu \mathrm{mol}, 0.5$ equiv) DBU or $15.4 \mathrm{mg}$ (49.3 $\mu \mathrm{mol}, 0.5$ equiv) ${ }^{t} \mathrm{Bu}-\mathrm{P}_{1}$ base (tert-Butylimino-tri(pyrrolidino)phosphorane) and $26.4 \mathrm{mg}(99.2 \mu \mathrm{mol}, 1.0$ equiv) pentamethylimidazolium iodide pre-A were dissolved in $5 \mathrm{~mL}$ of $\mathrm{d}_{3}$-acetonitrile and subsequently divided into three samples. ${ }^{1} \mathrm{H}$ NMR measurements were conduct after 20 minutes and 24 hours after mixing for every sample.
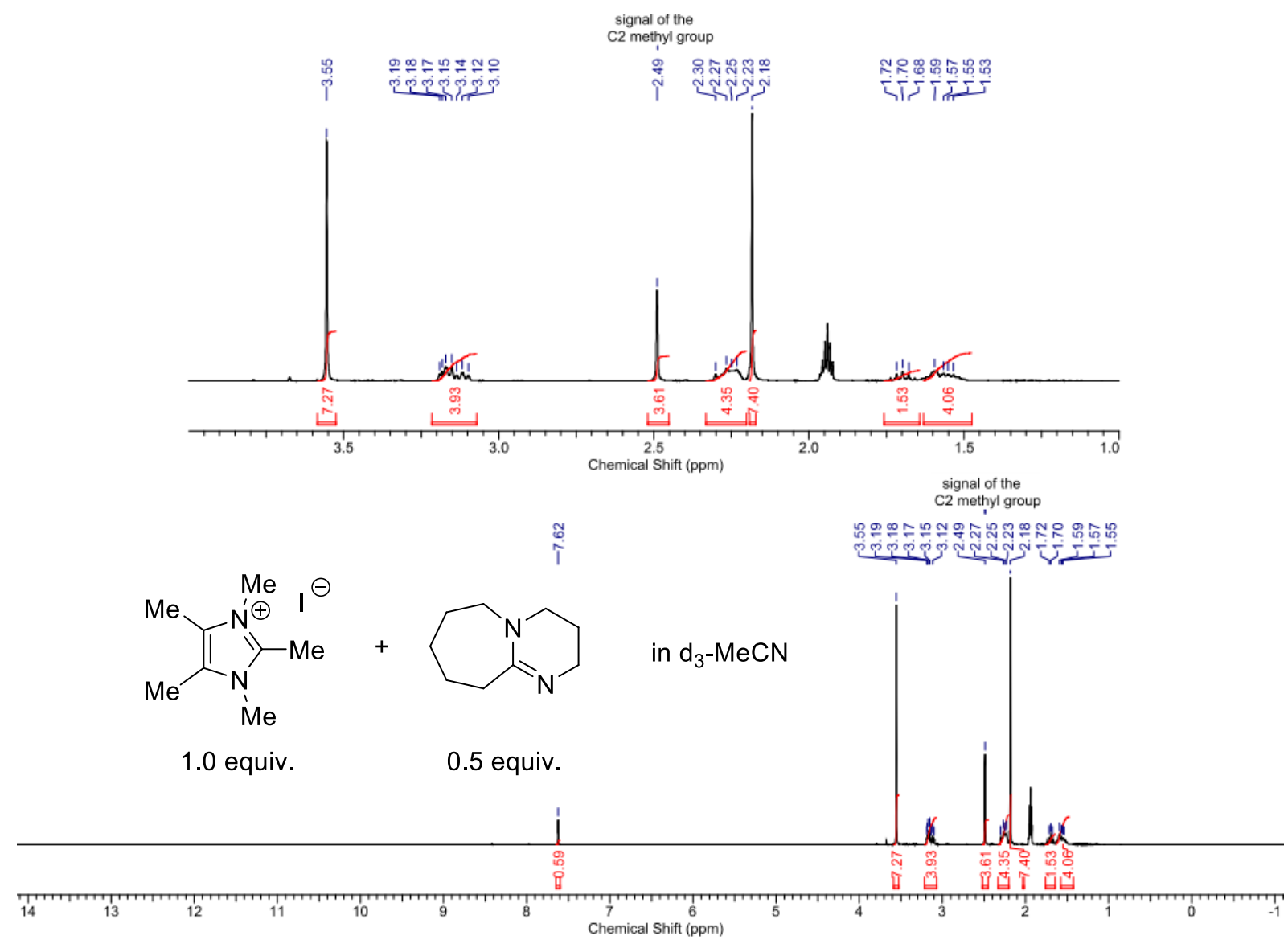

The NMR signal of the C2 methyl group remained unchanged when DBU was used to deprotonate the NHO precursor salt. Furthermore, no protonation of DBU was observed, which proves that $\mathbf{A}$ exhibits a higher $\mathrm{p} K_{\mathrm{a}}$ than DBU. 

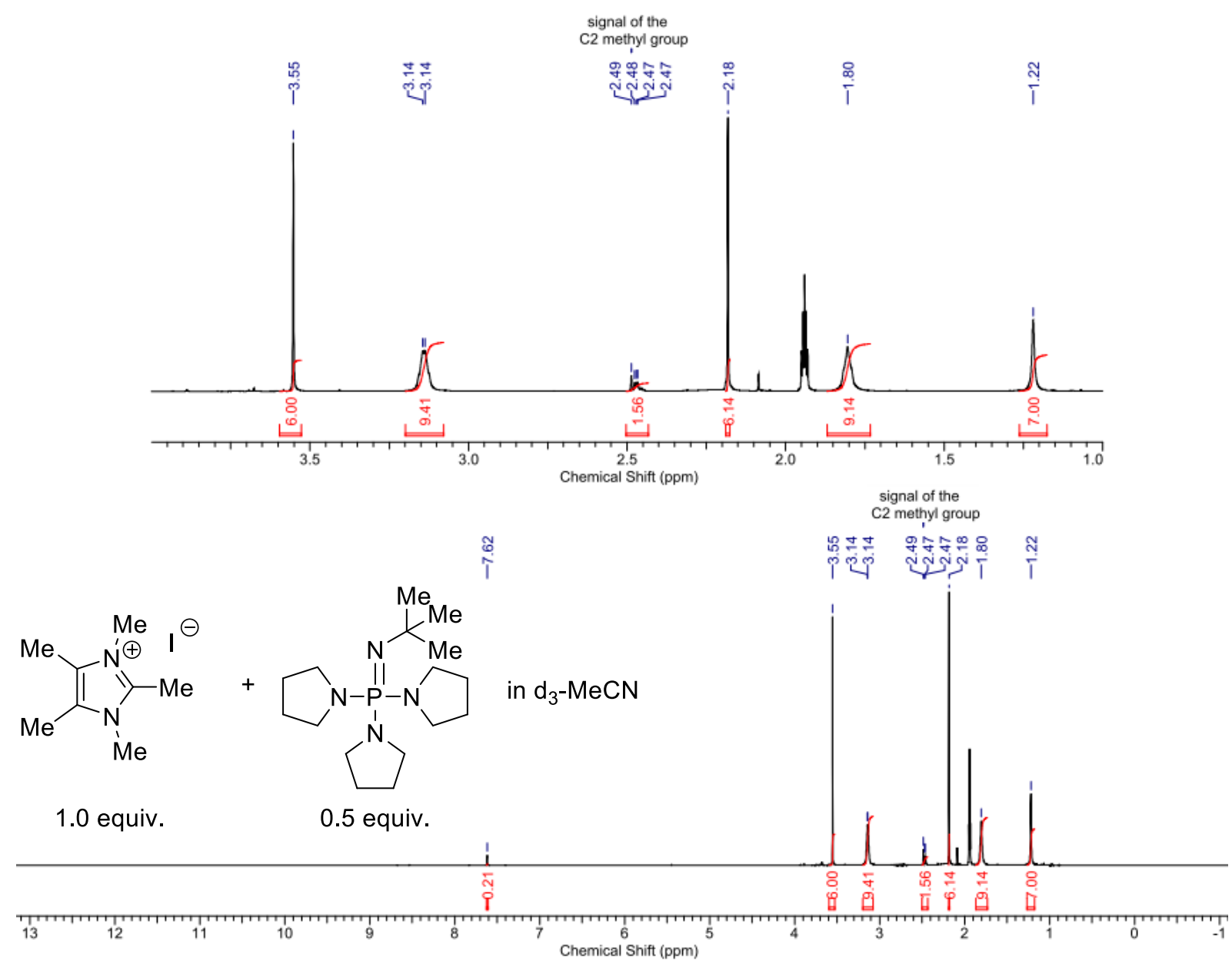

When the ${ }^{t} \mathrm{Bu}-\mathrm{P}_{1}$ base was used for the deprotonation of pre-A the signal of the $\mathrm{C} 2$ methyl group disappeared. However, the starting deuterium exchange between the deuterated solvent and the free NHO resulted in a multiplet signal at a chemical shift from 2.47-2.49 ppm. Thus we conclude that the ${ }^{t} \mathrm{Bu}-\mathrm{P}_{1}$ base is a stronger base than the representative NHO A. Therefore, the $\mathrm{p} K_{\mathrm{a}}$ of $\mathbf{A}$ lies within the range of $24.3\left(\mathrm{p} K_{\mathrm{a}}[\mathrm{DBU}]\right)$ and $28.4\left(\mathrm{p} K_{\mathrm{a}}\left[{ }^{t} \mathrm{Bu}-\mathrm{P}_{1}\right]\right)$. 


\section{Spectra}

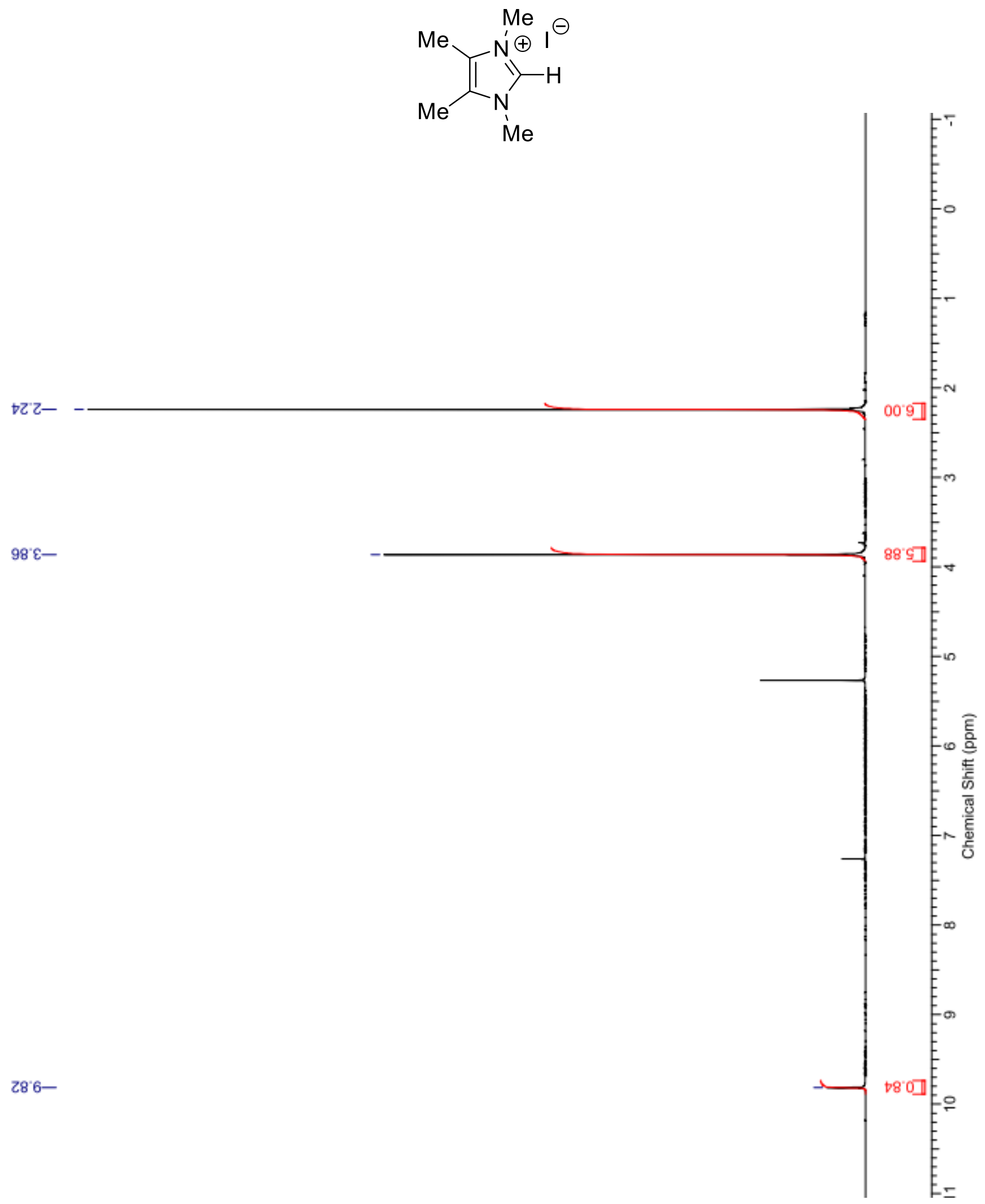


28'8-

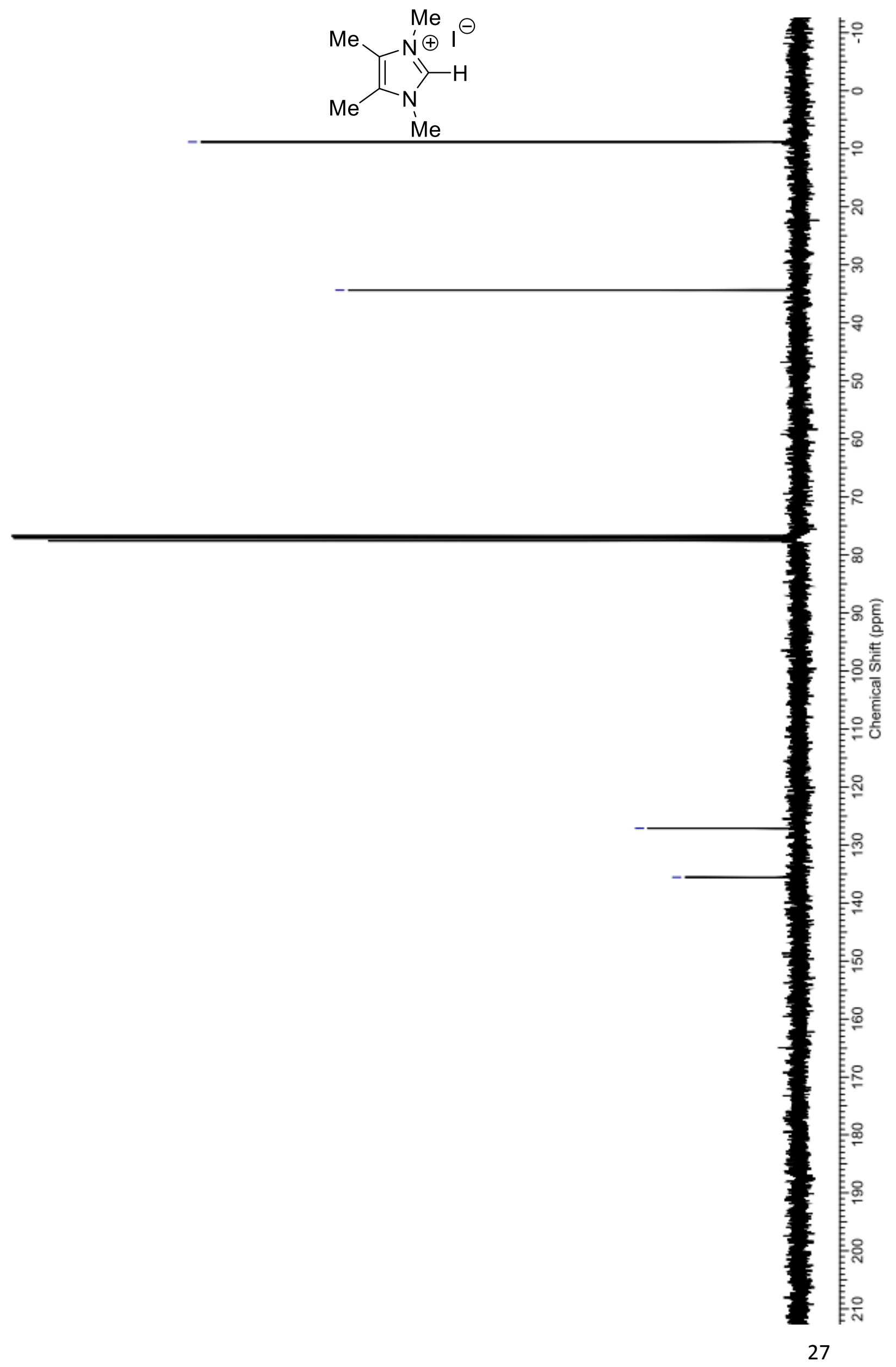




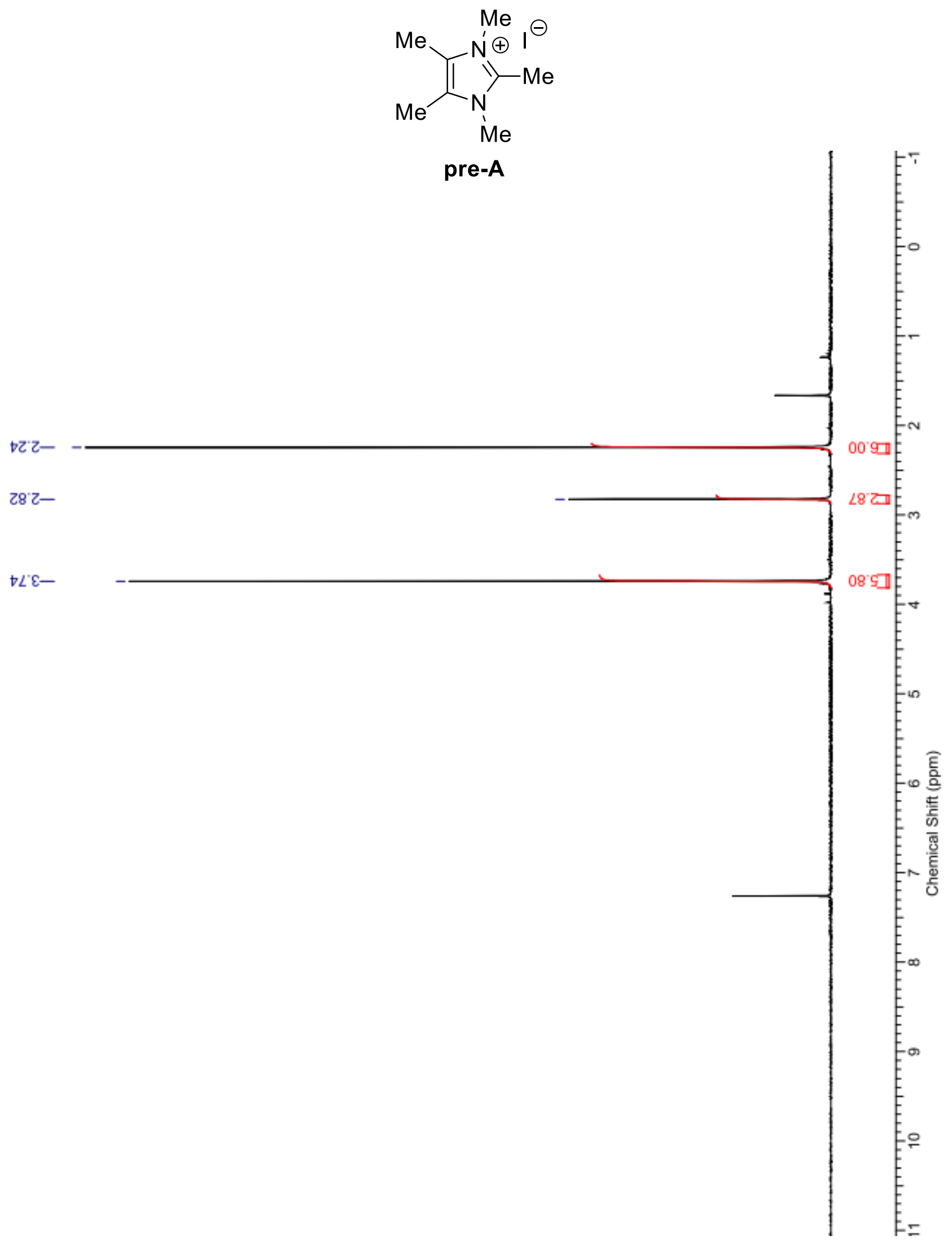


6Z'6-
\&L'Zl-

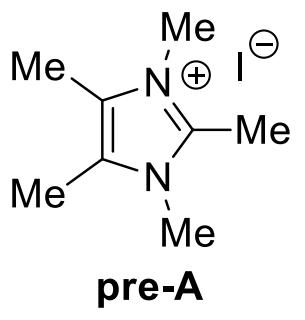

ธะระ-

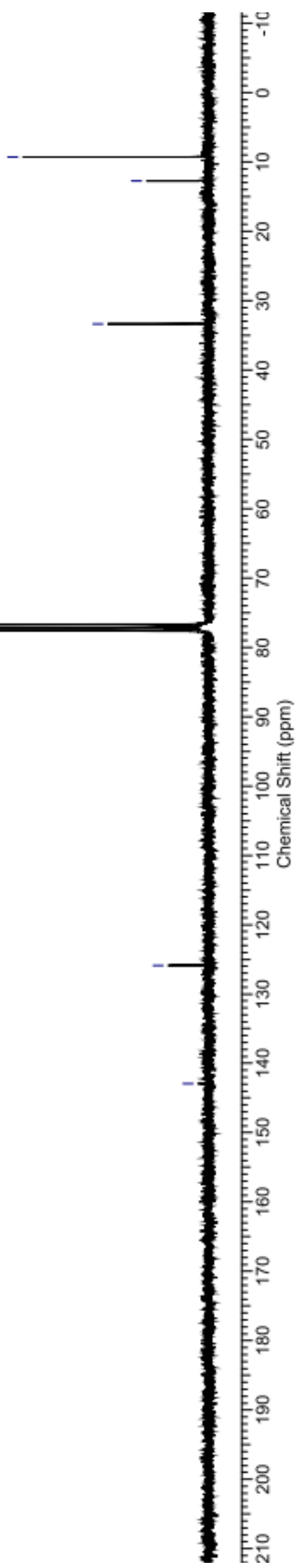




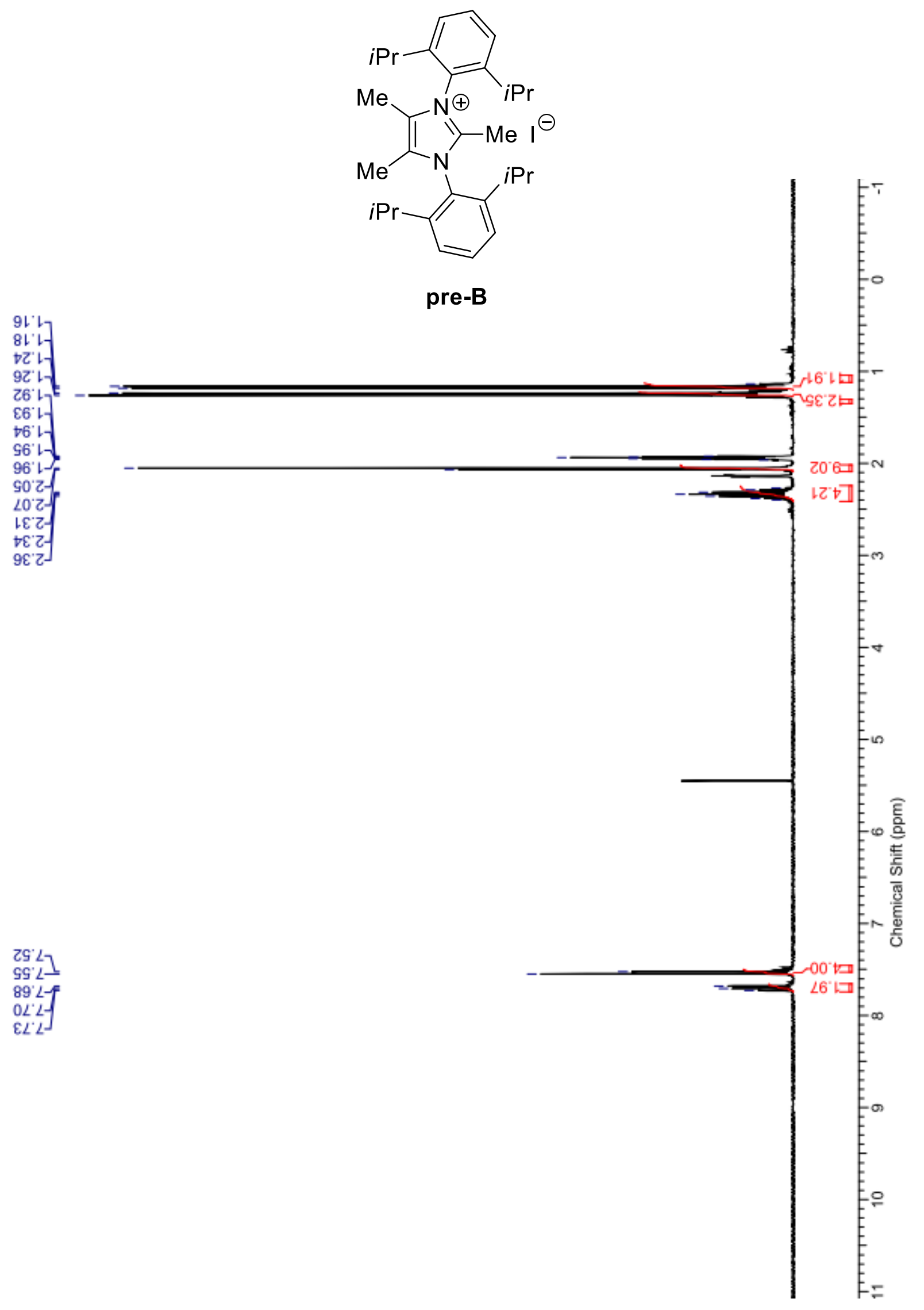


$\mathrm{CrO}-$

9l'Zl-

$9 \mathrm{~L}^{\prime} \mathrm{z}-$

$\angle 0^{\circ} 6 \mathrm{Z}$ -

28'SZ!

$00^{\circ} \mathrm{LL}$ -

Z8'8Z1

เ8'Z\&L-

$\angle Z^{\prime} \varepsilon D L_{-}$ 6Z'St-<smiles>[M]C1=C(C(C)C)N(c2c(C(C)C)cccc2C(C)C)C(C)=C(C(C)C)N1c1c(C(C)C)cccc1C(C)C</smiles>

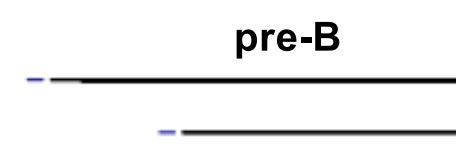




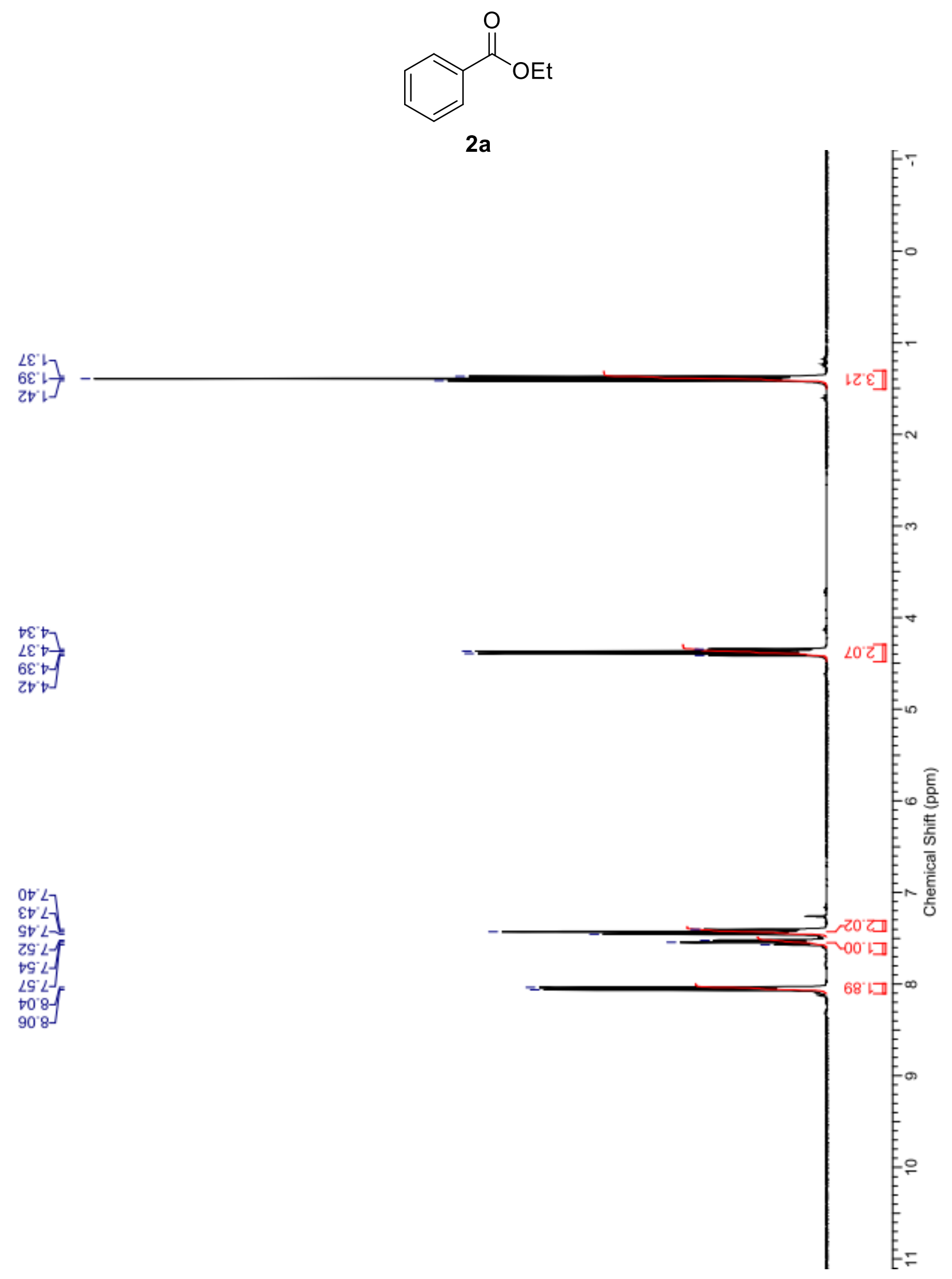


ยท゙เレ-<smiles>CCOC(=O)c1ccccc1</smiles>

So'เ9-

$59^{\circ} 62$ 工

$29^{\circ} 0 \mathrm{EL}-$

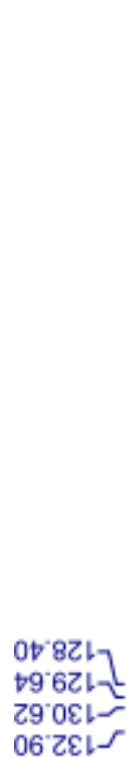

SL'991- 
<smiles>CCCCOC(=O)c1ccccc1</smiles>

2b

$1 \varepsilon^{-} t$
$\varepsilon \varepsilon^{-} t-1$
$s \varepsilon^{-} t$

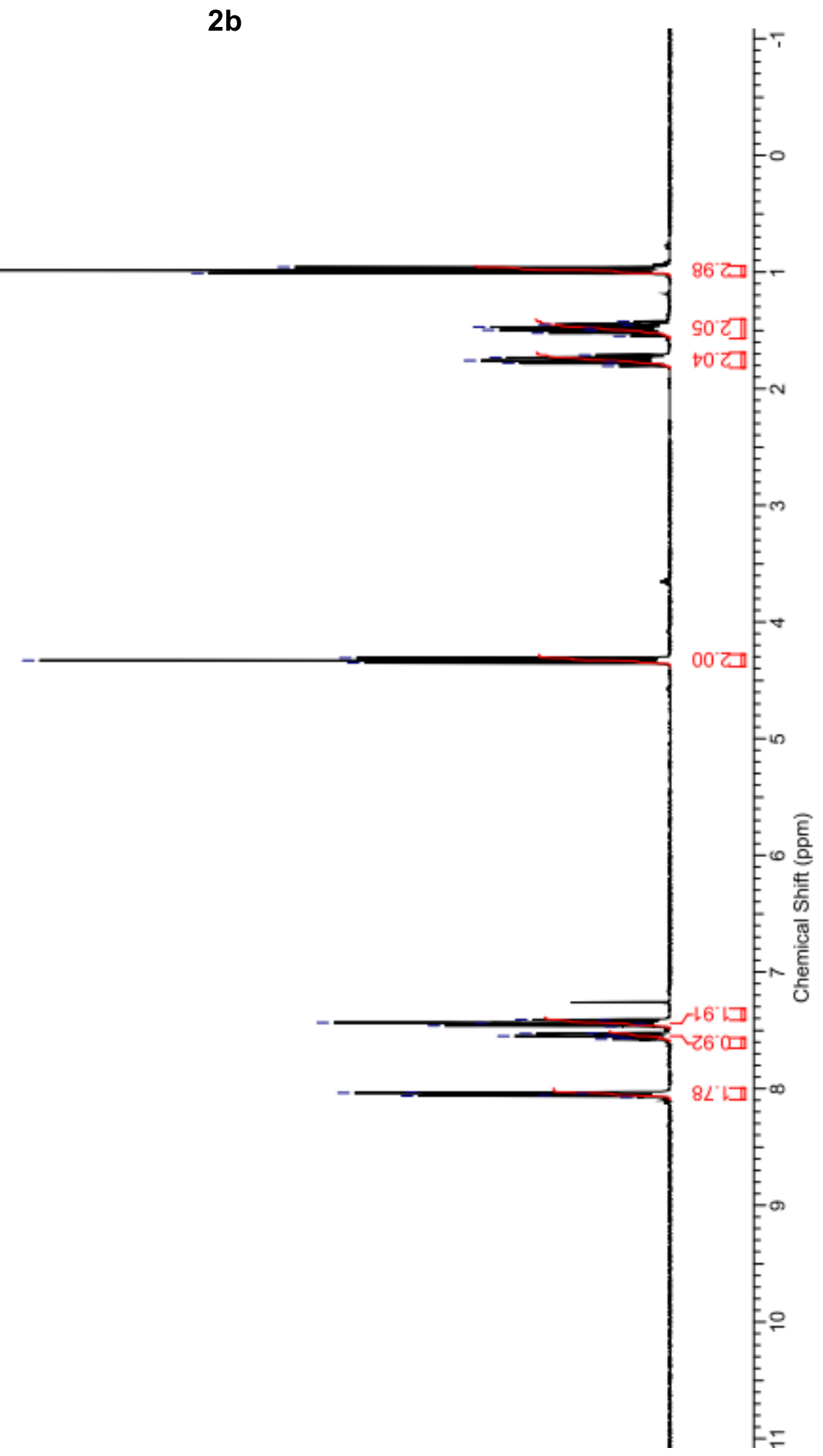




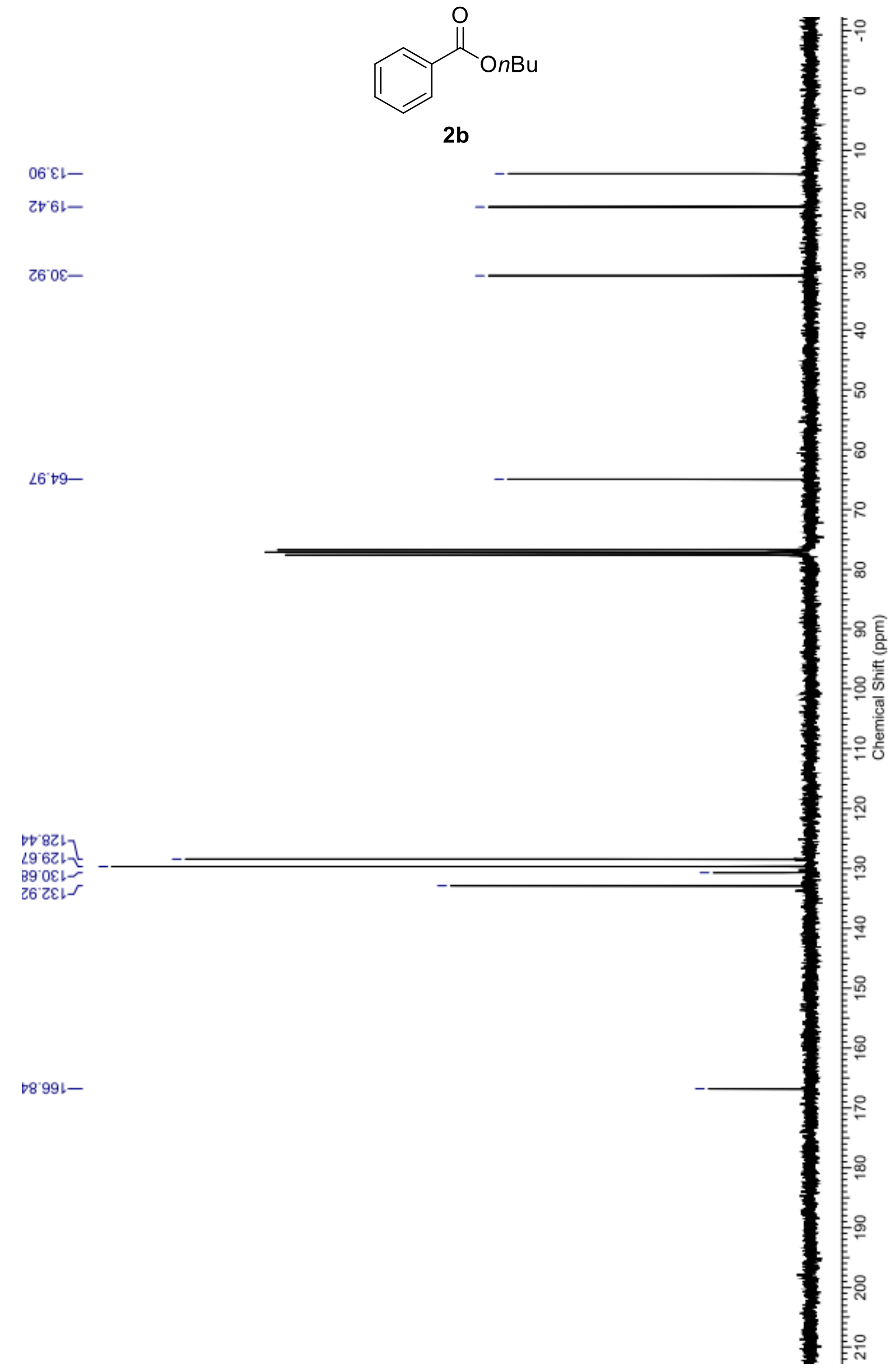




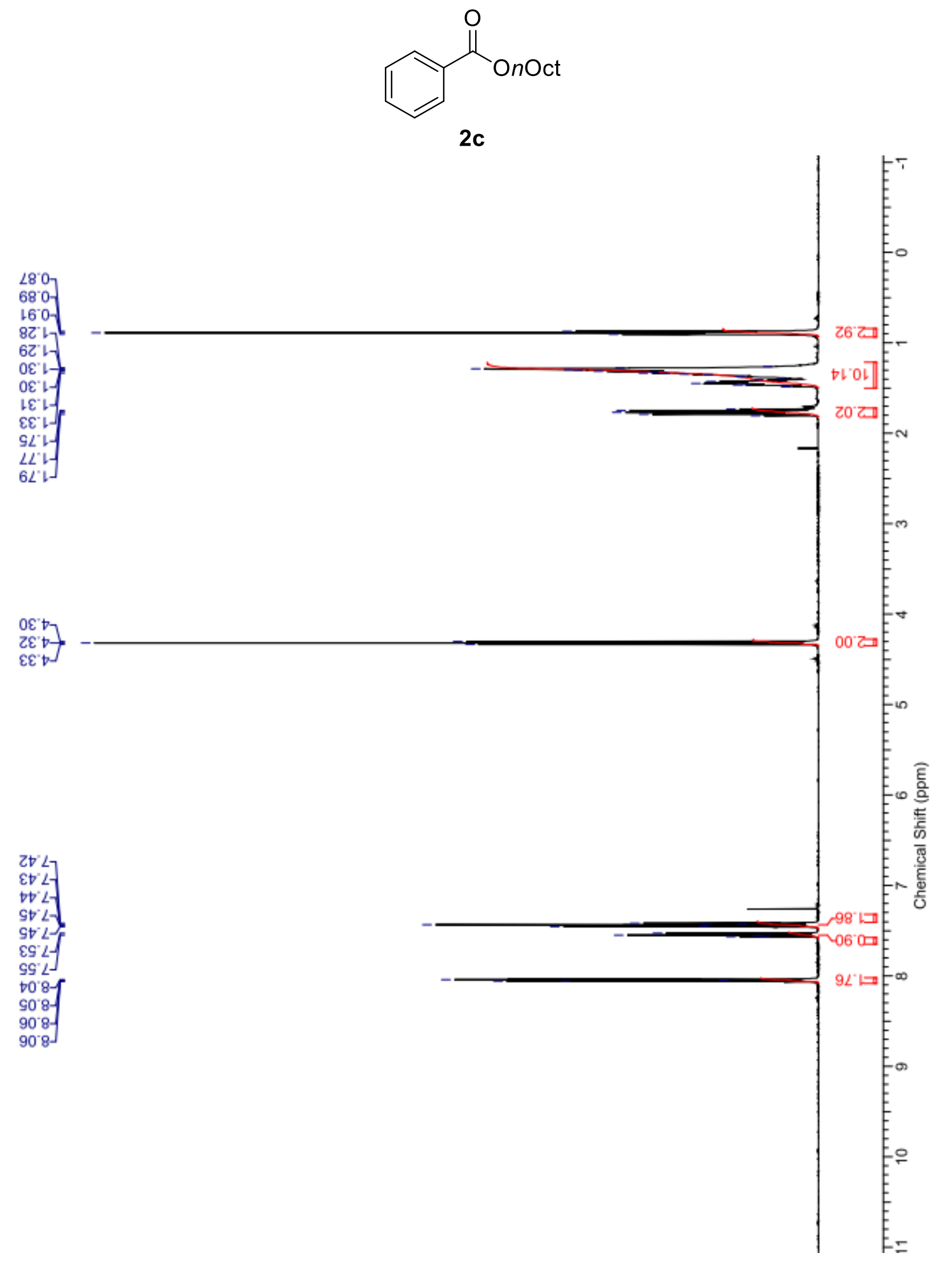


เฉ

LLZC

81 '92

$98^{\circ} 82$

$8 \varepsilon^{*} 62^{-5}$

$9299-$

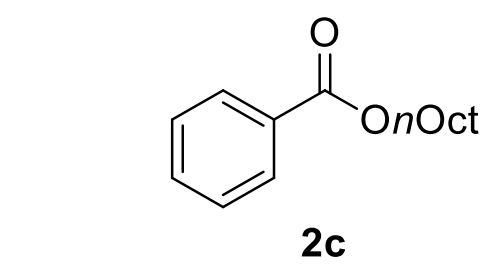

$861 \varepsilon$

Z $8 \mathrm{Zl}$

99.62 म

B9.0ع -

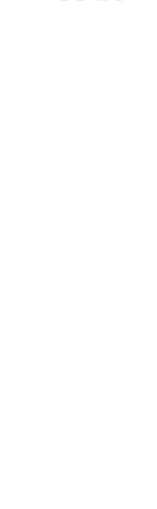

08'991- 


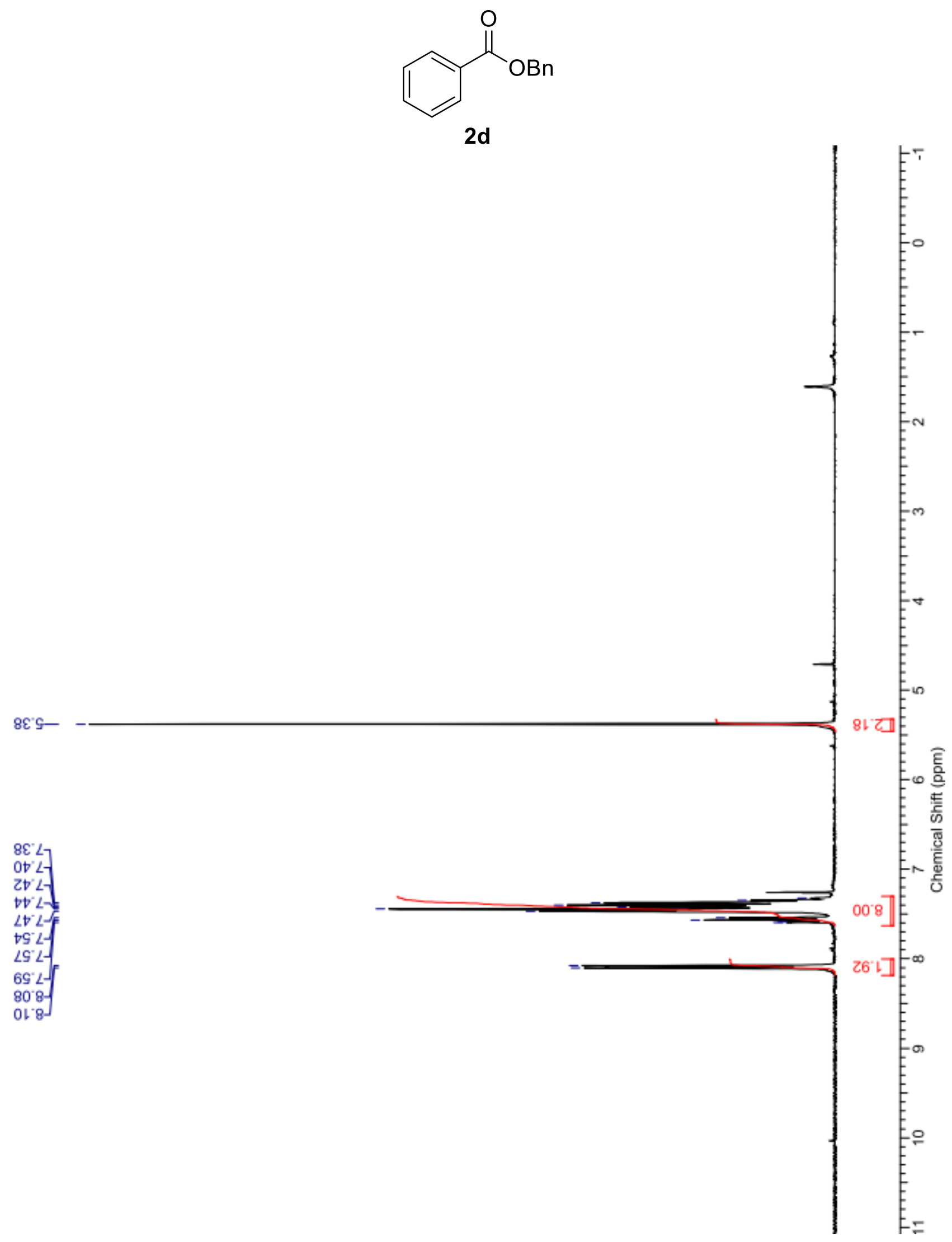




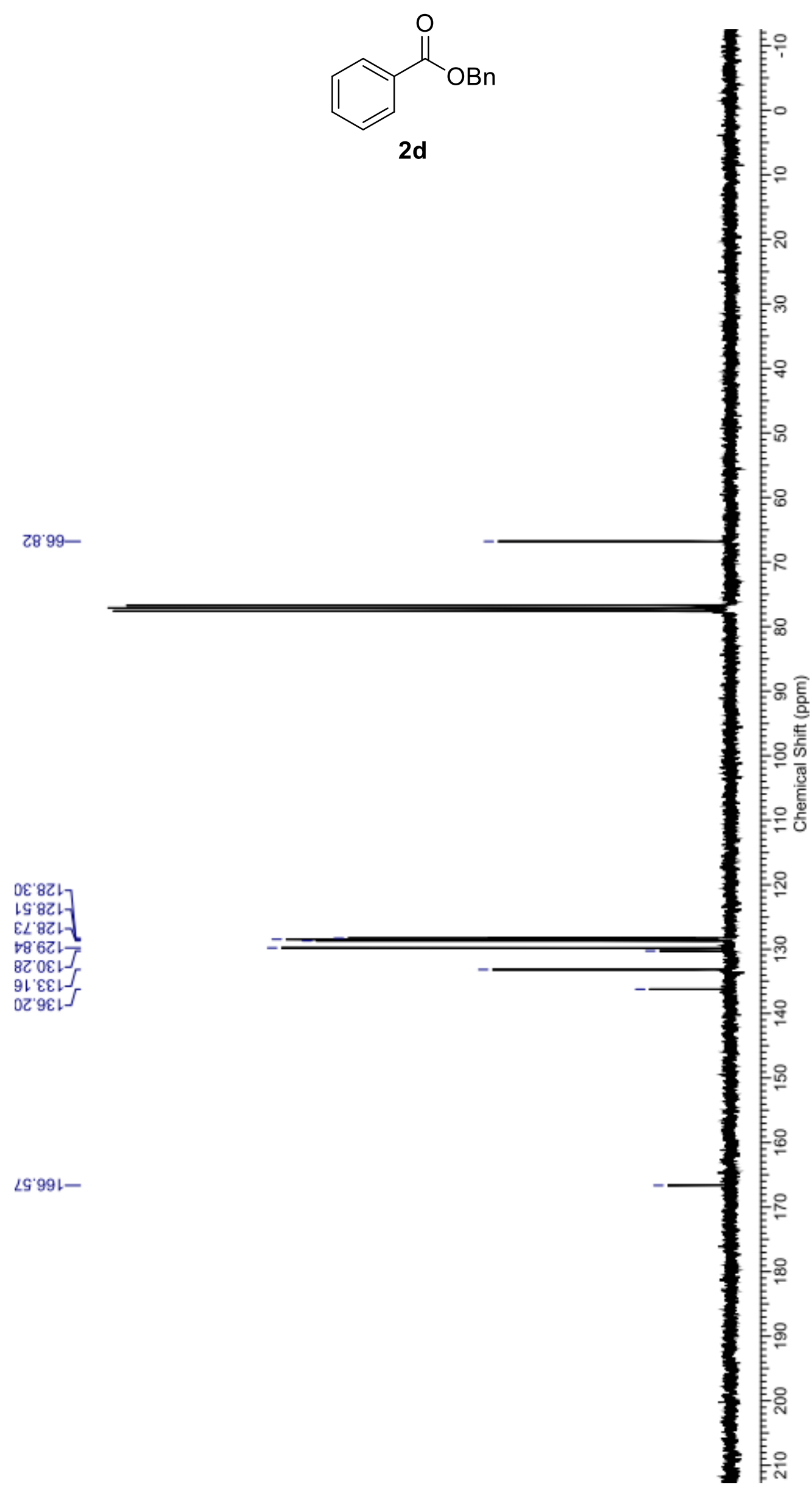




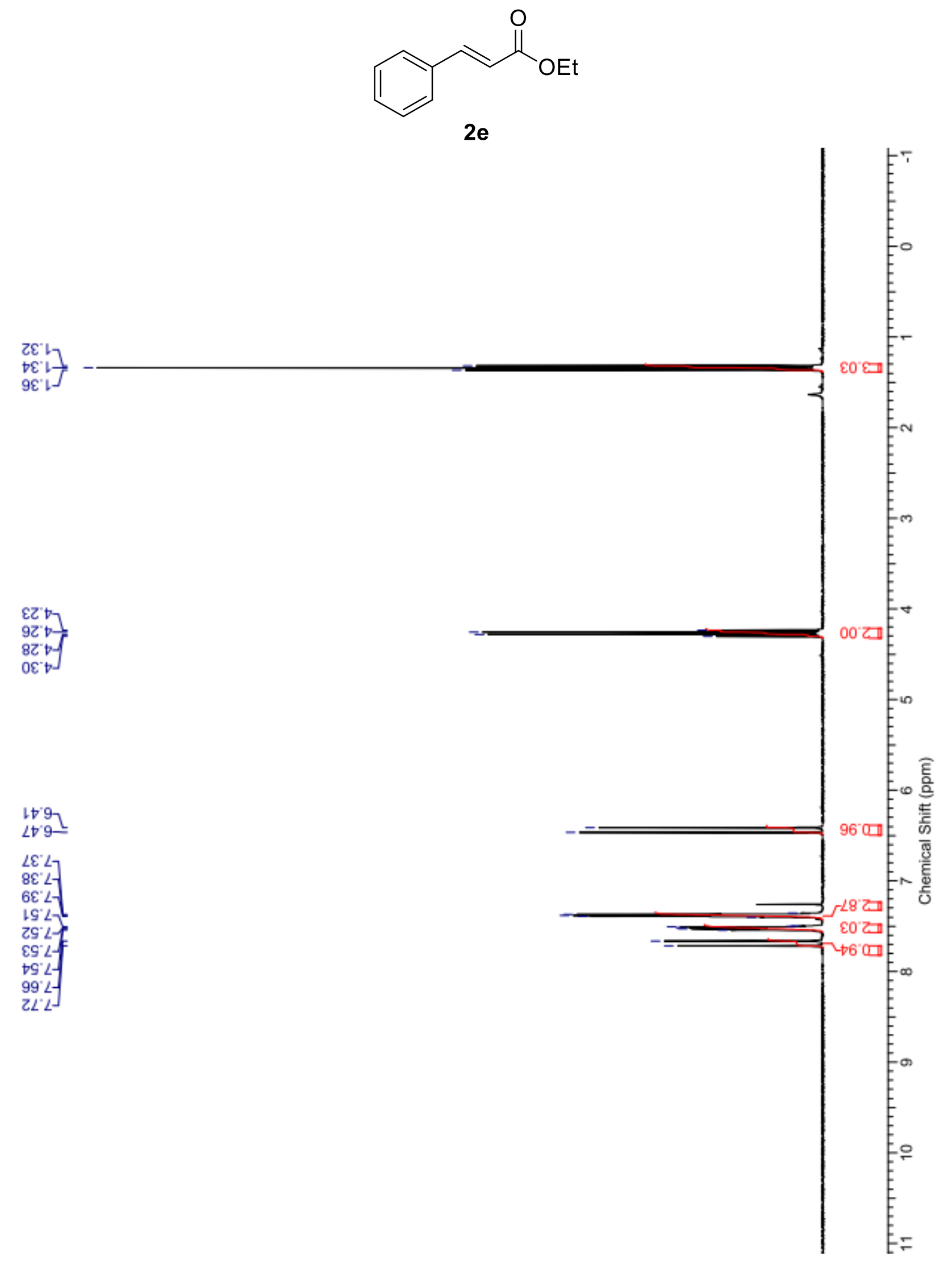




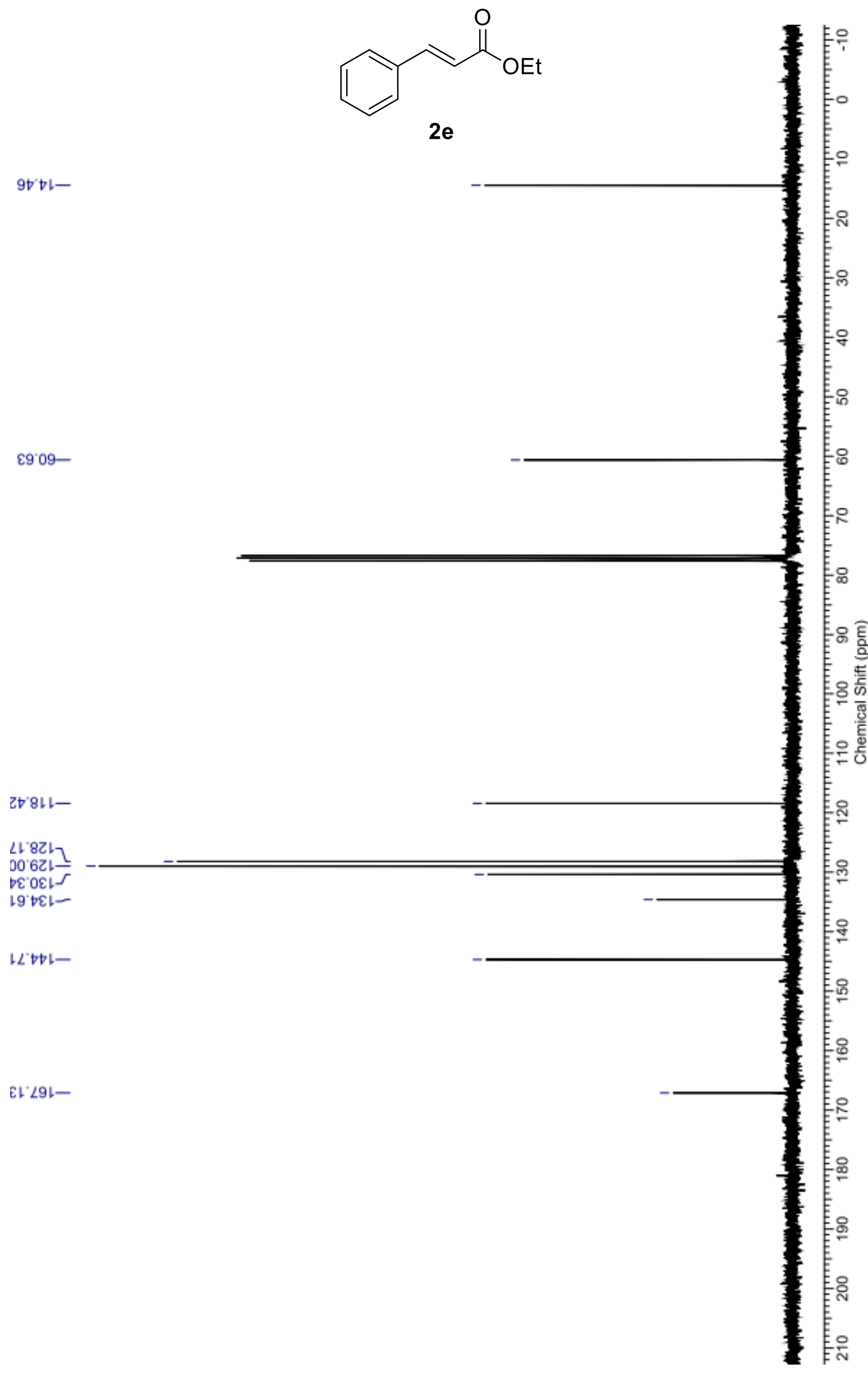

41 


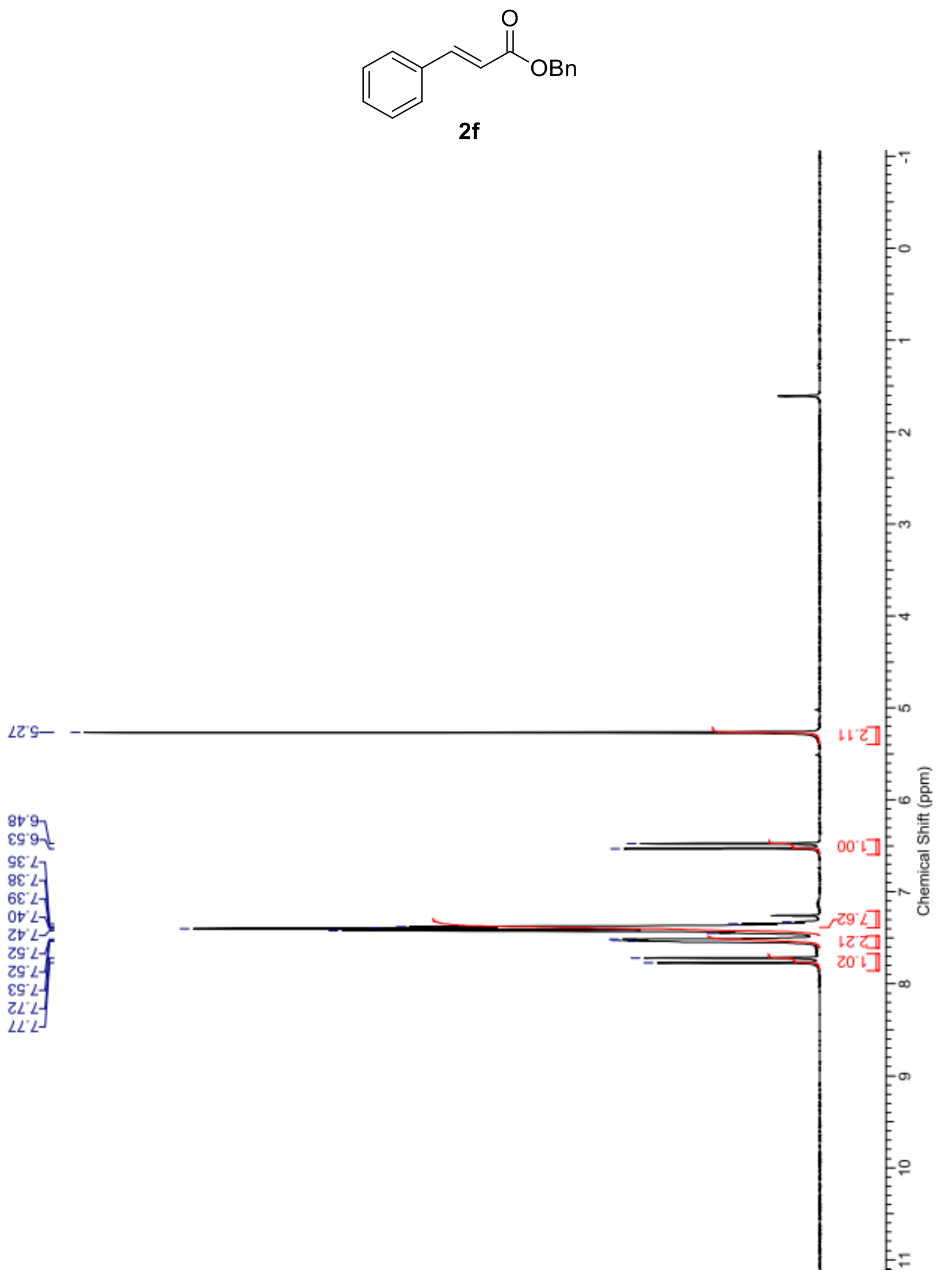




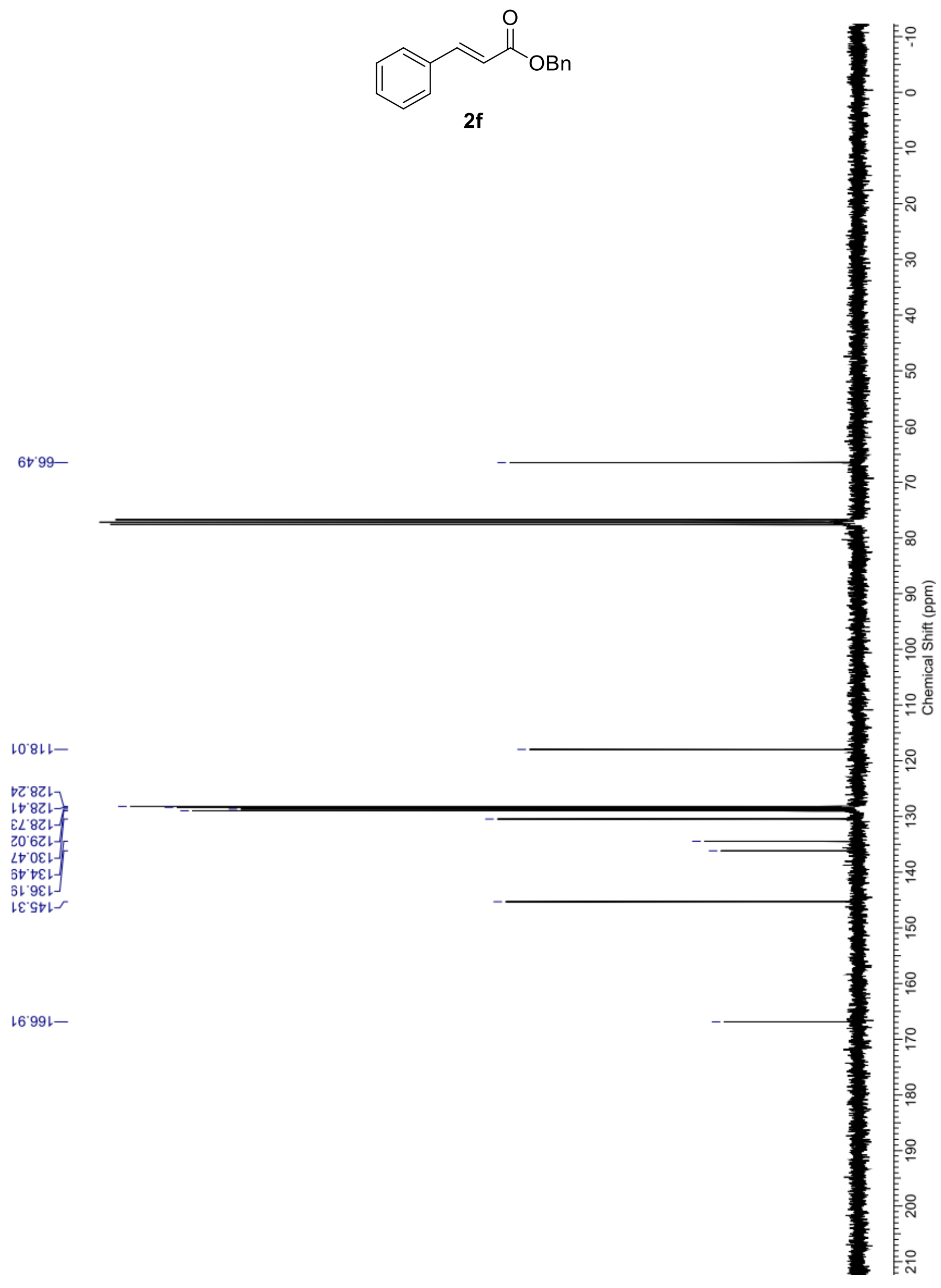




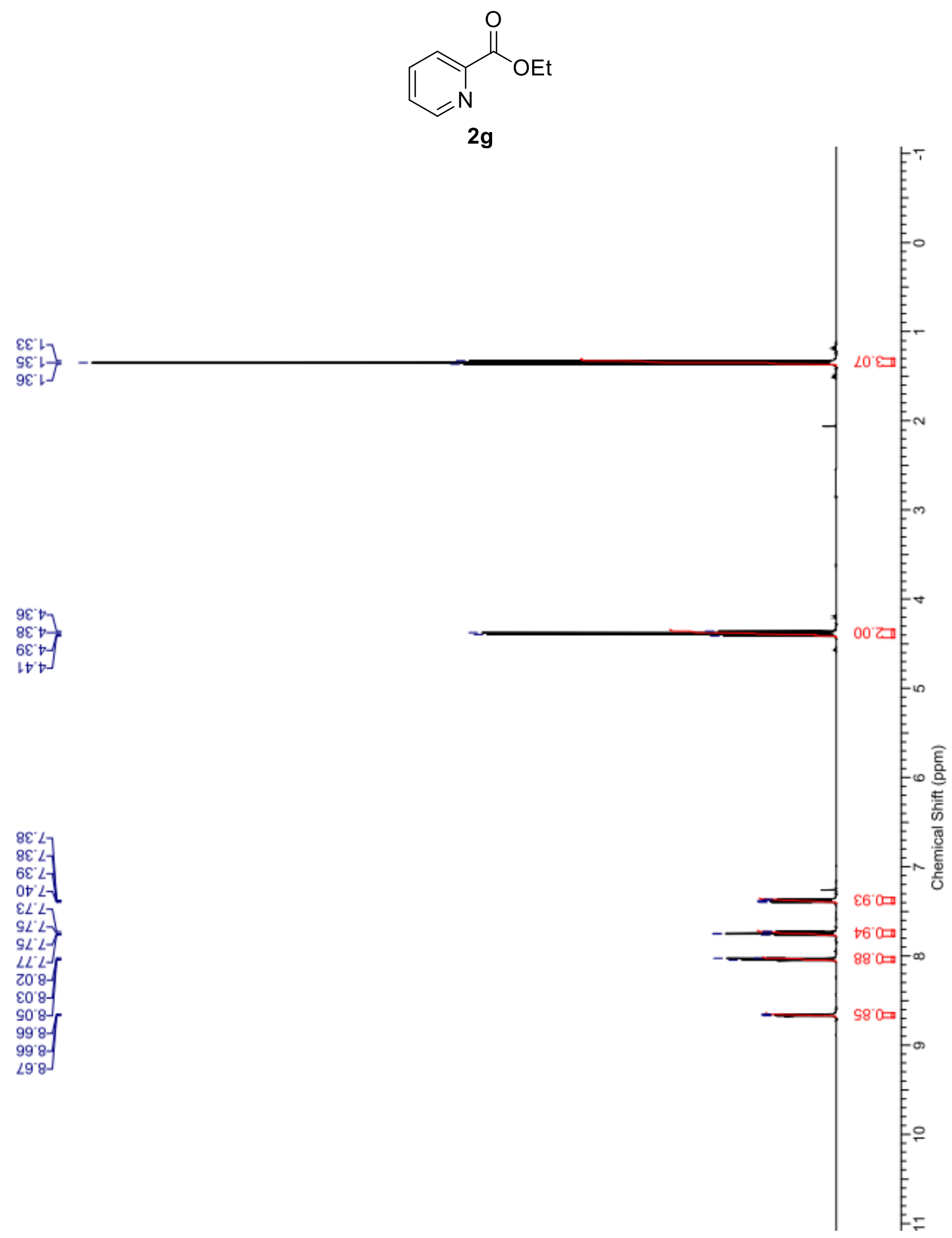




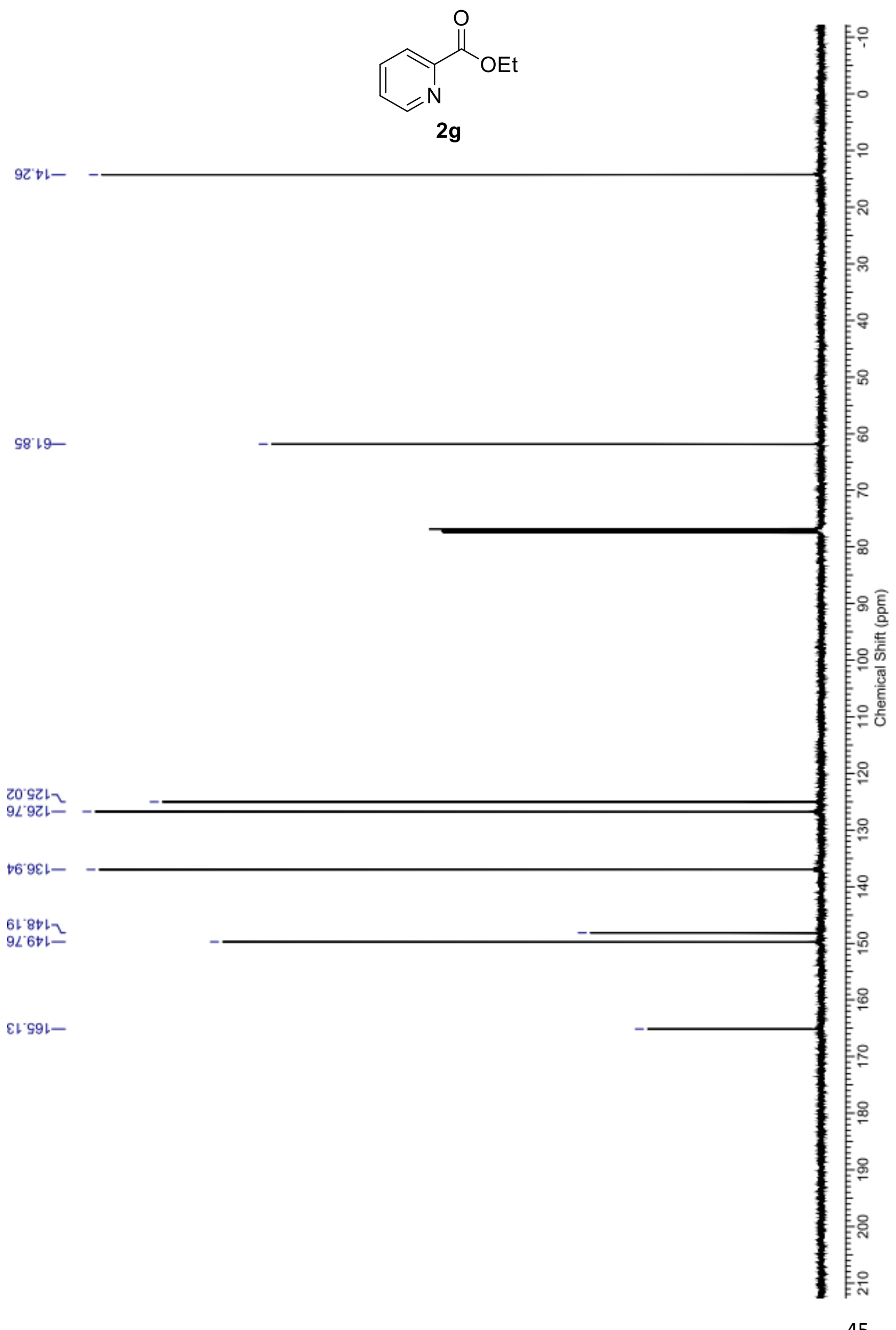




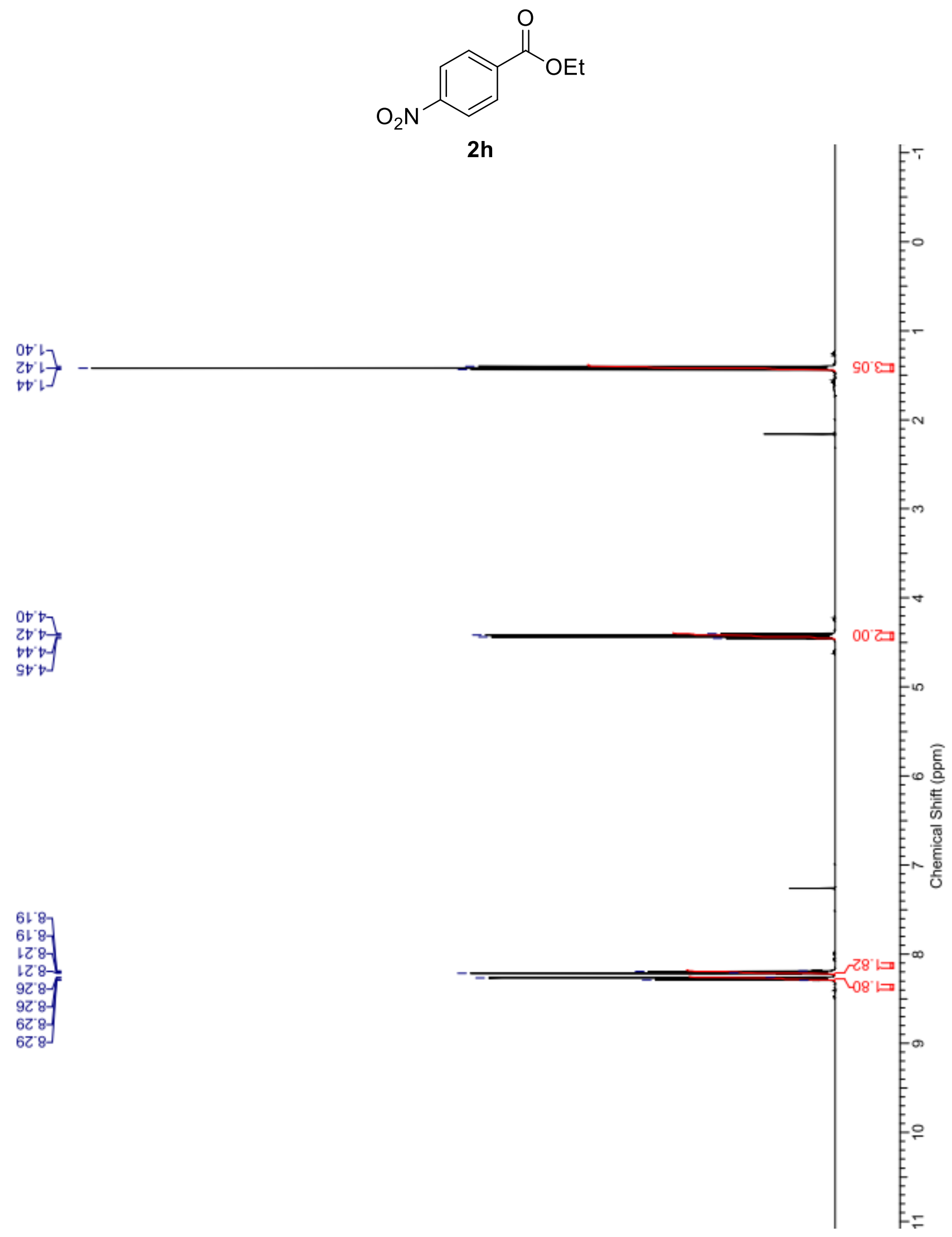


sEเ

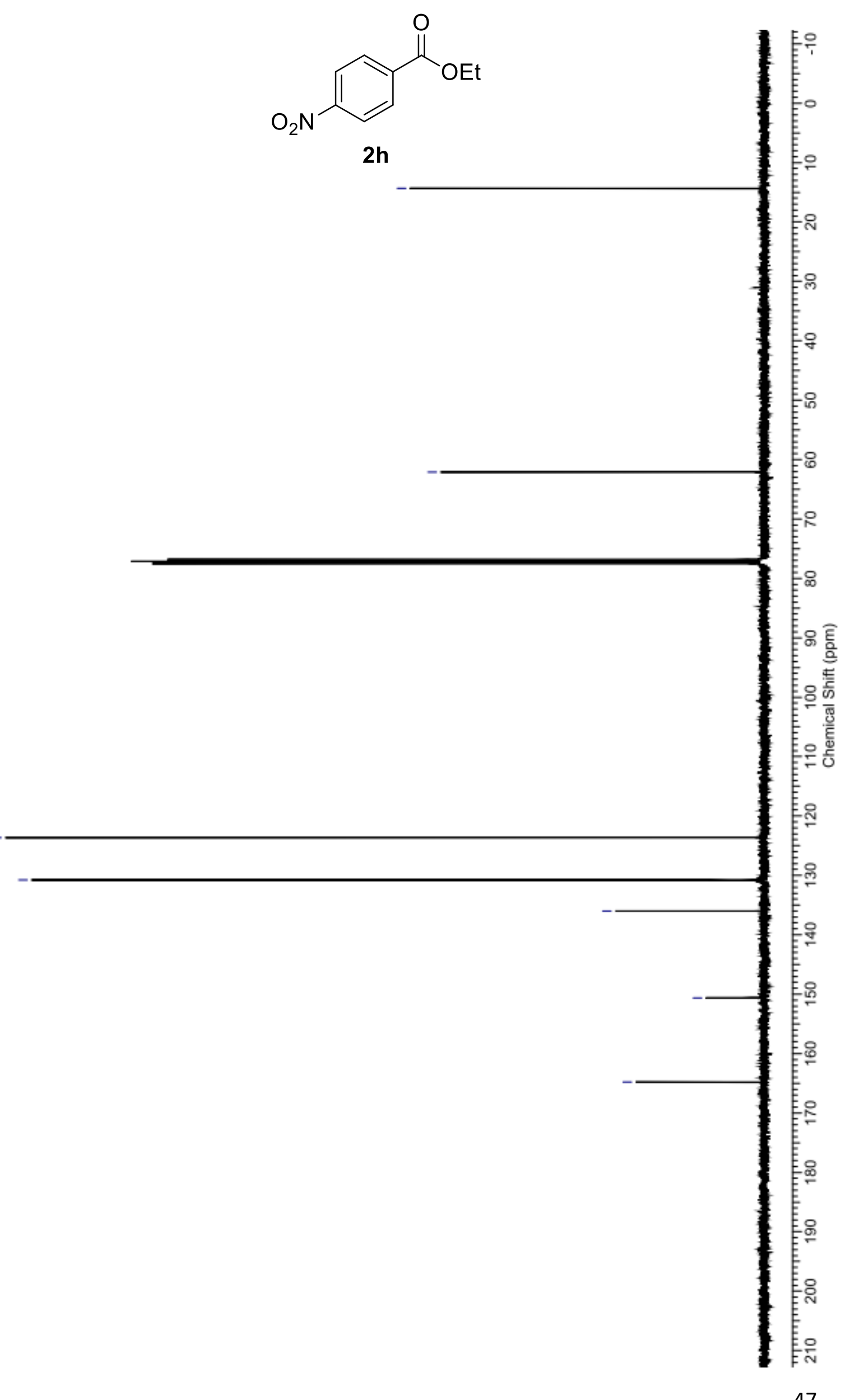

8079

29091-

$18^{\circ}+91-$

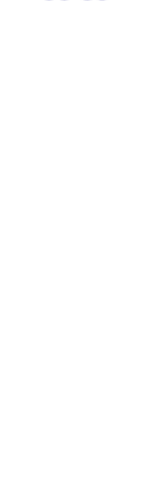

乙9.

6LOㄴ-

66's\&ᄂ-

18 


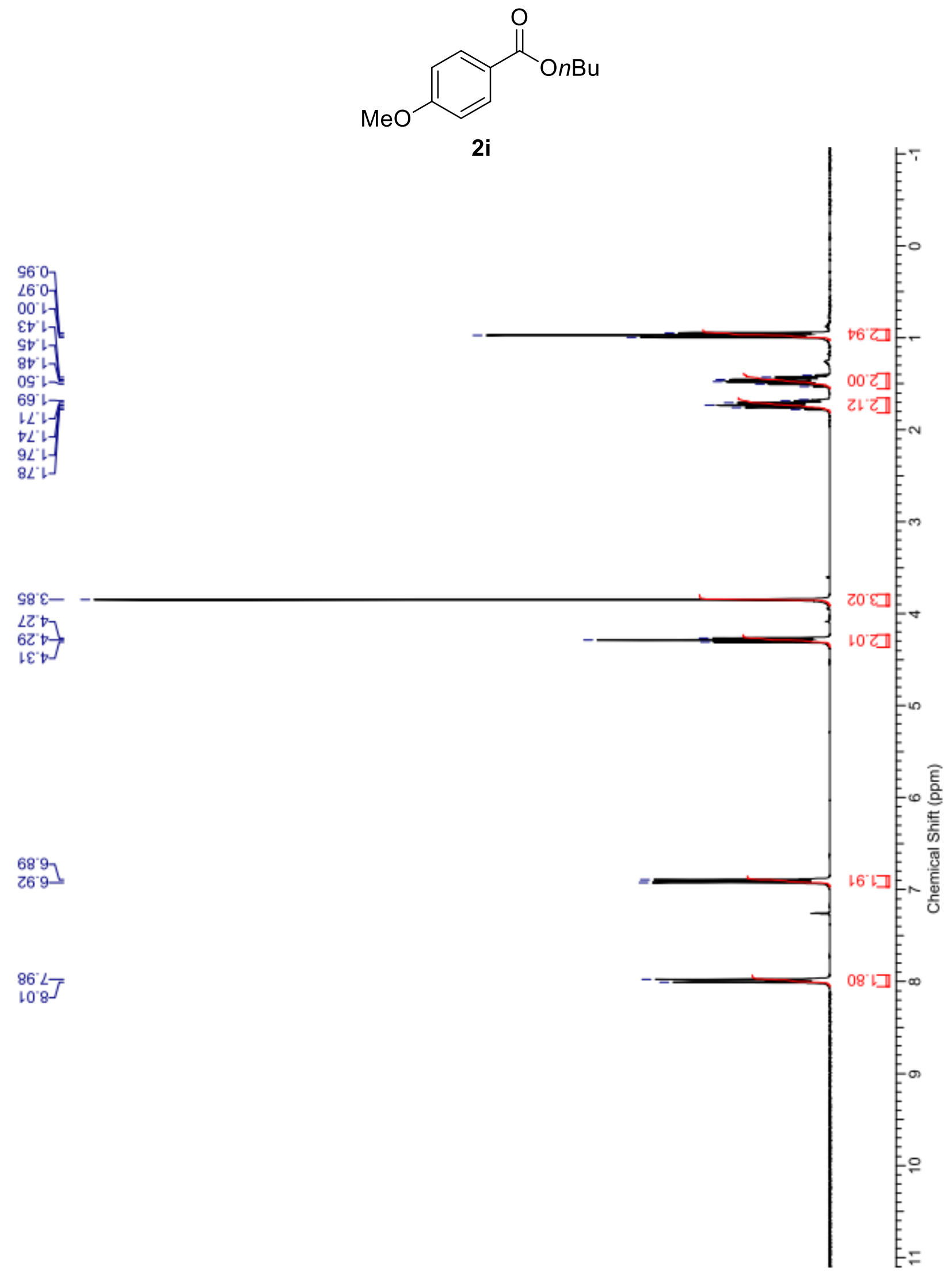


$06^{\circ} \varepsilon 1-$
乙ฟ'6L-
$\angle 6^{\circ} 0 \varepsilon-$

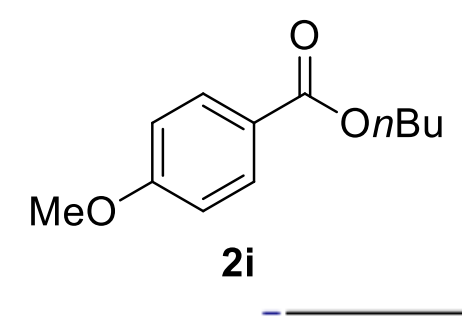

\&s'ss-

$59+9-$

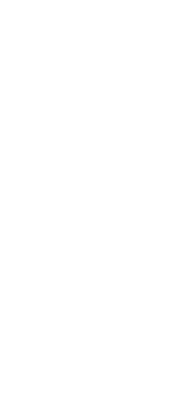

น9ยเレー

そเยฉเー

ร9"เยเ-

$\angle \varepsilon^{\prime} \varepsilon 91-$

89.99l-
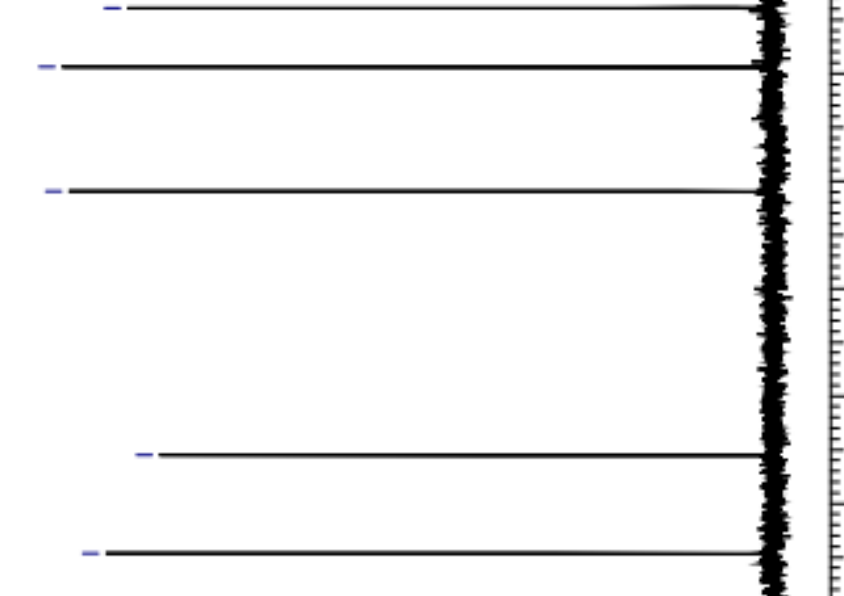

요

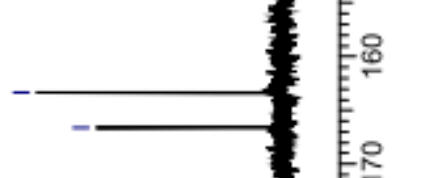




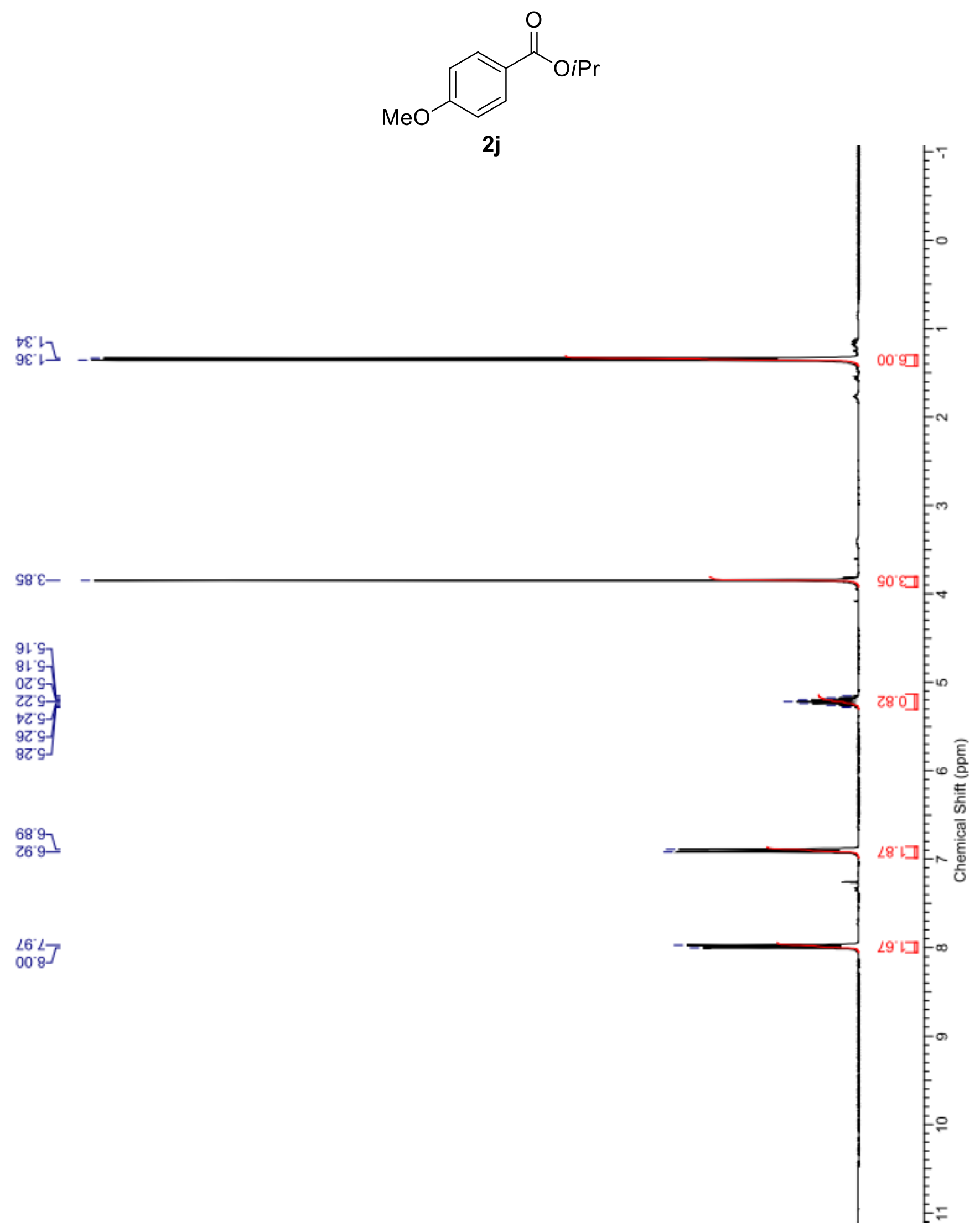




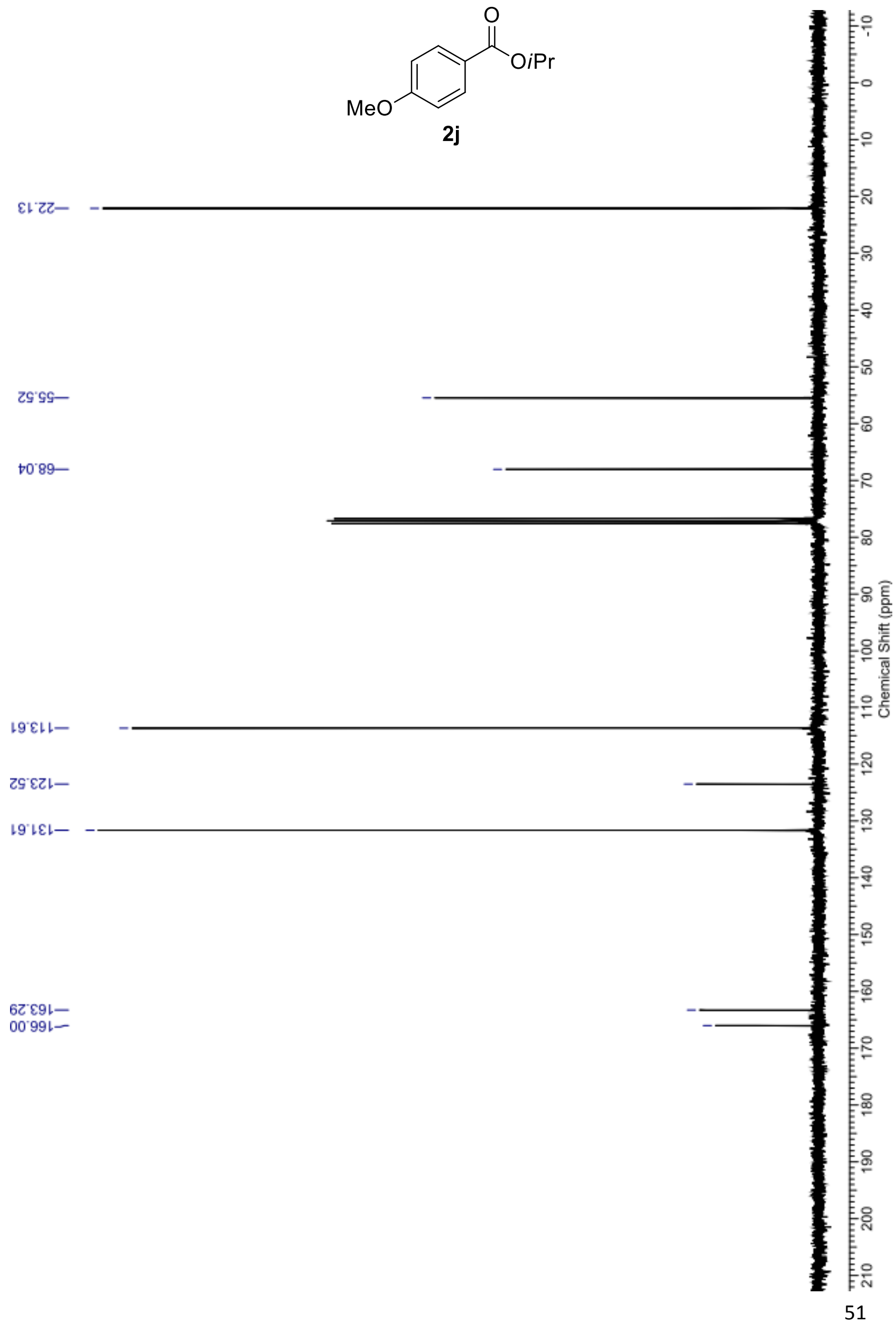



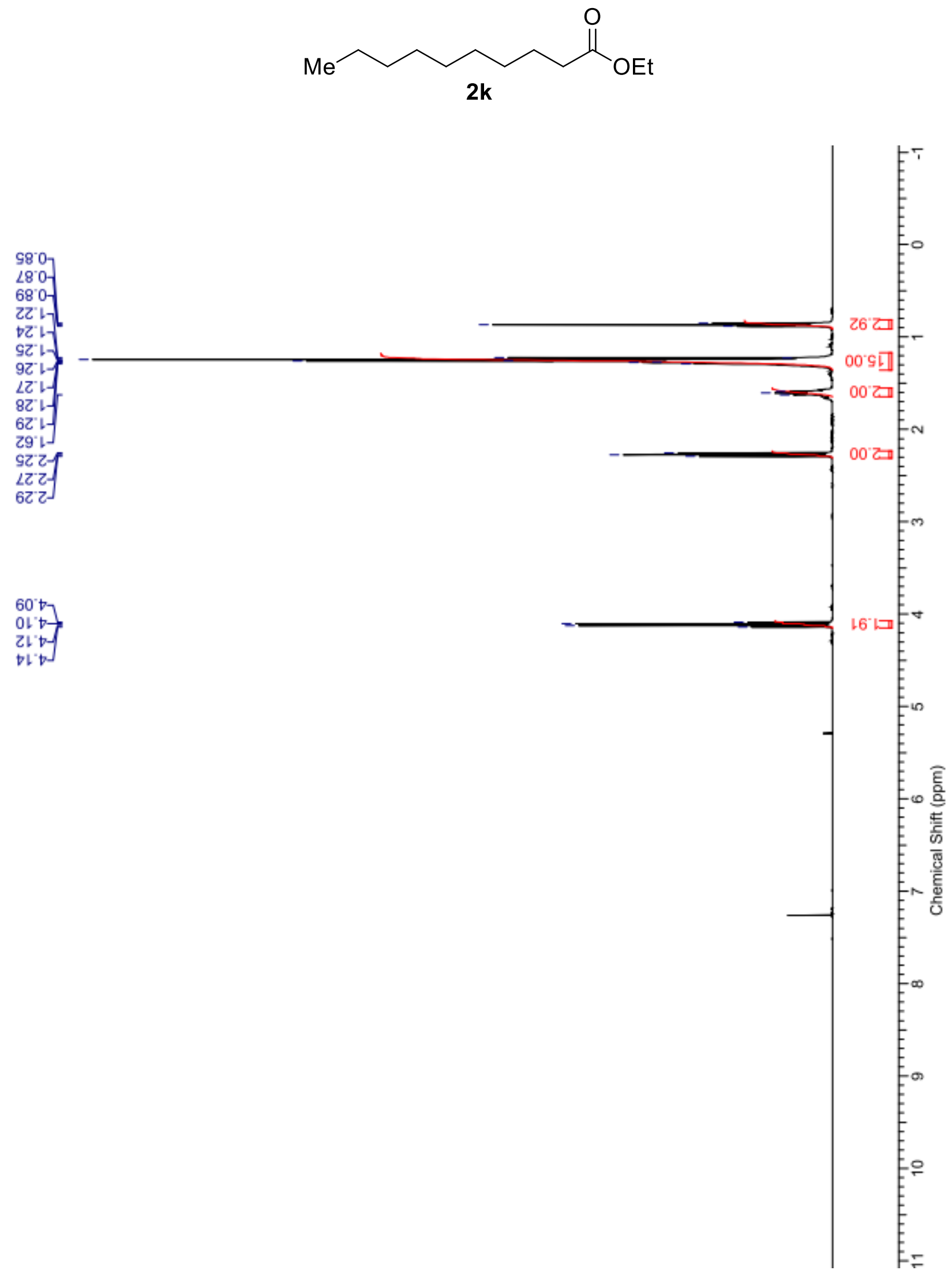
$z x^{\circ}+1$
$\left.8 \varepsilon^{\prime}+1\right]$

$08^{\prime} 2 z_{7}$

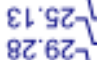

$6 \varepsilon^{\circ} 62-$

s.'62

66 ' เ

9209-

$60^{\circ} \nabla \angle L-$<smiles>CCCCCCCCCC(=O)OCC</smiles>
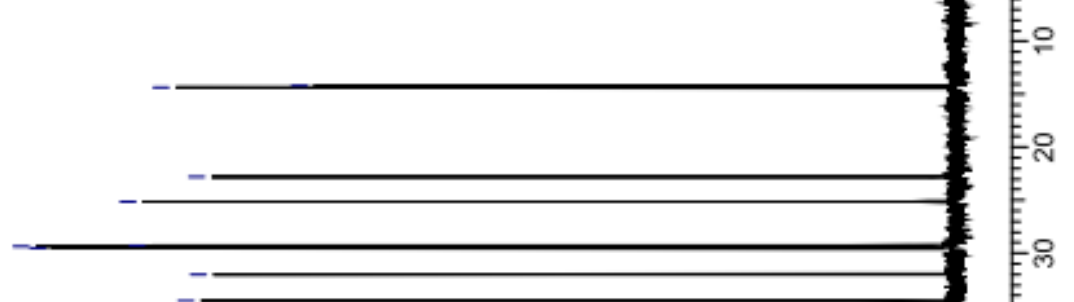

$-$

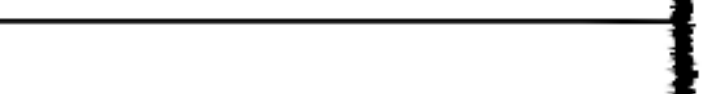


<smiles>O=C(CCCCCBr)OCCc1ccccc1</smiles>

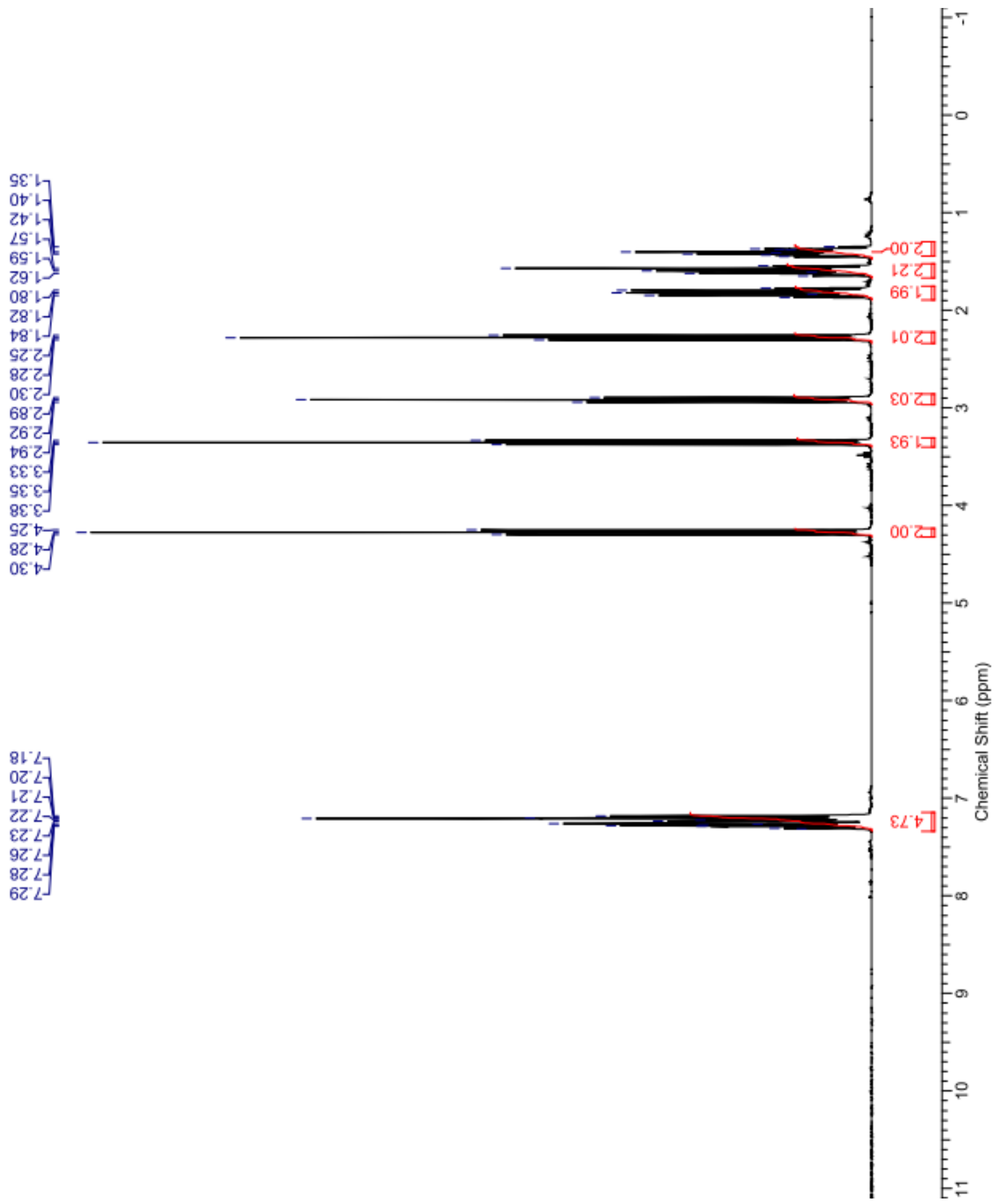




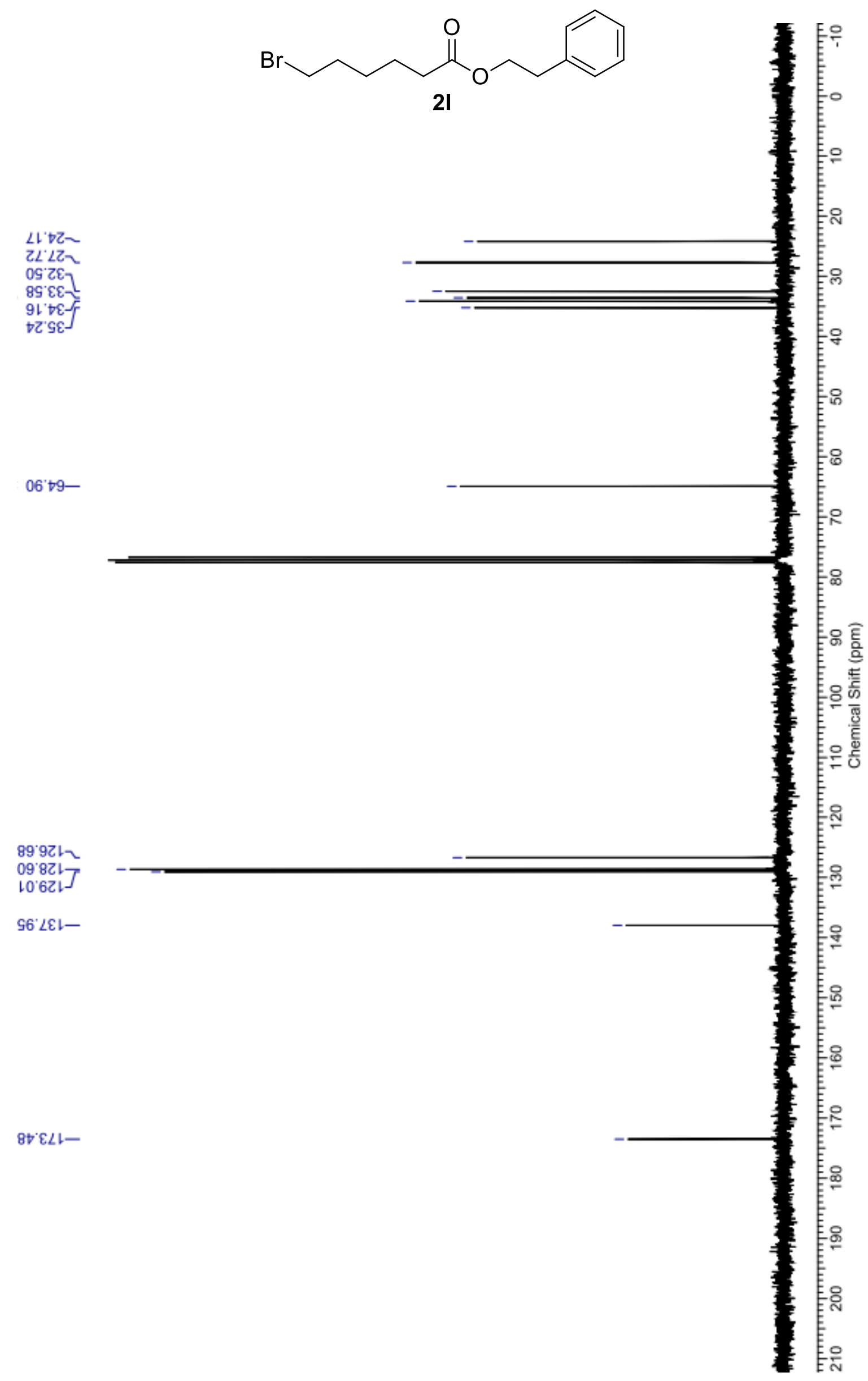



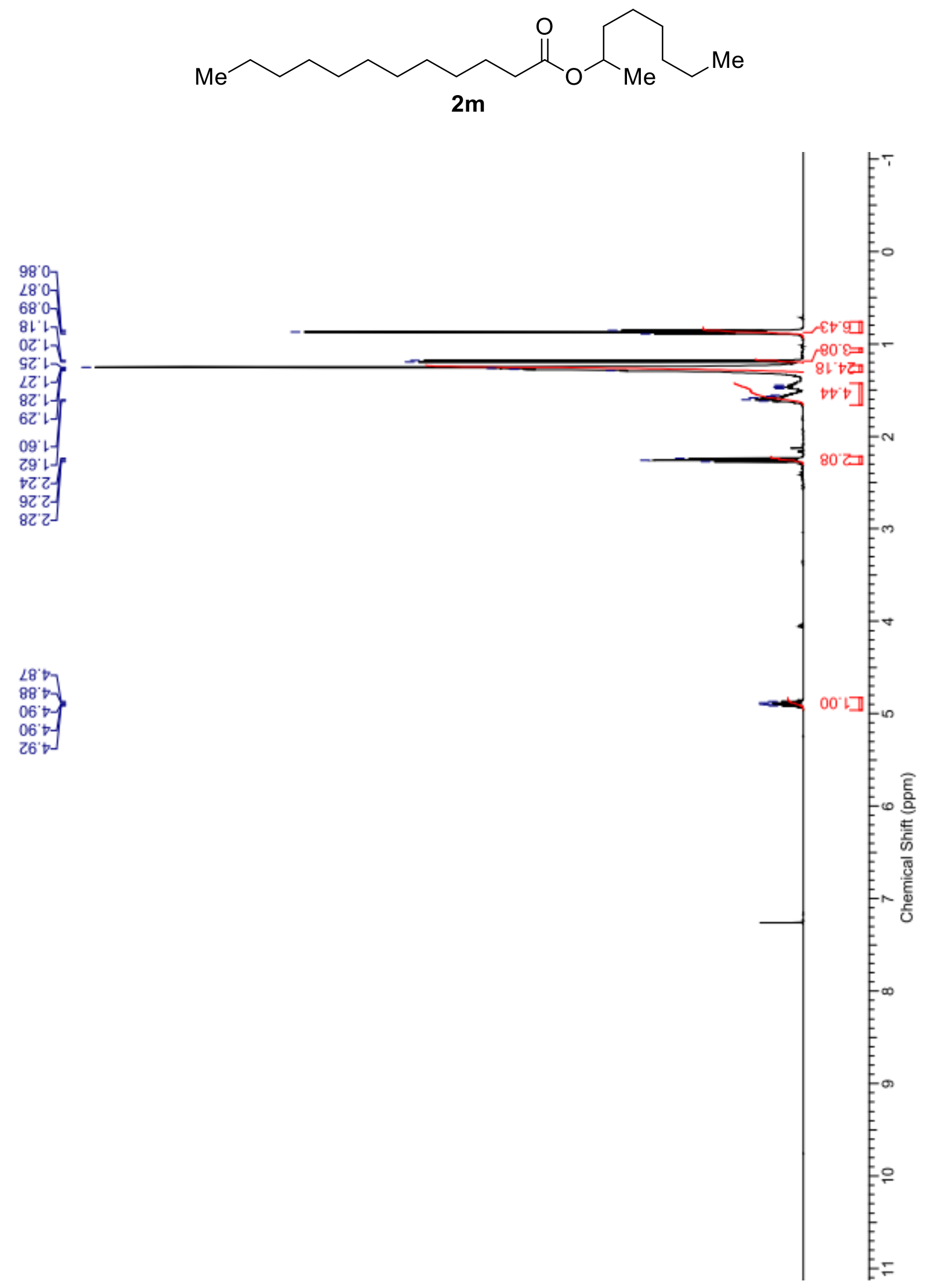

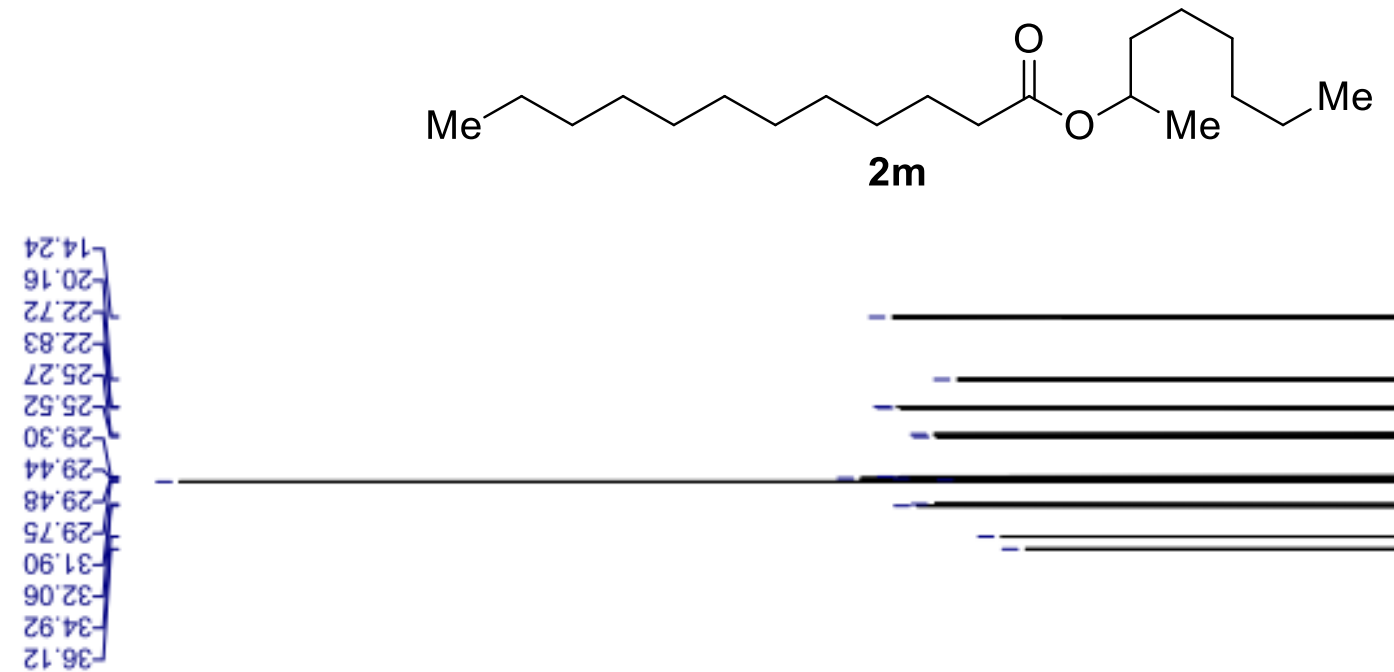

$S^{\circ} 0 L-$

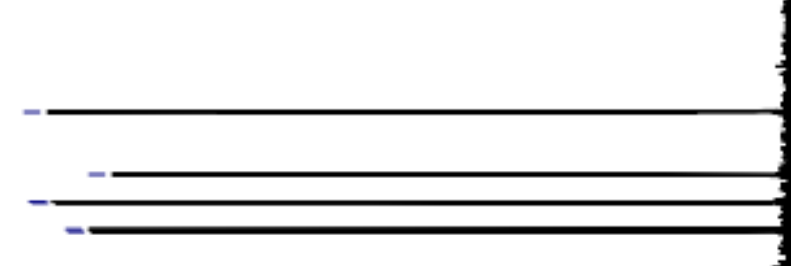


<smiles>CCCCCCCCCCCC(=O)OC1CCCCC1</smiles>

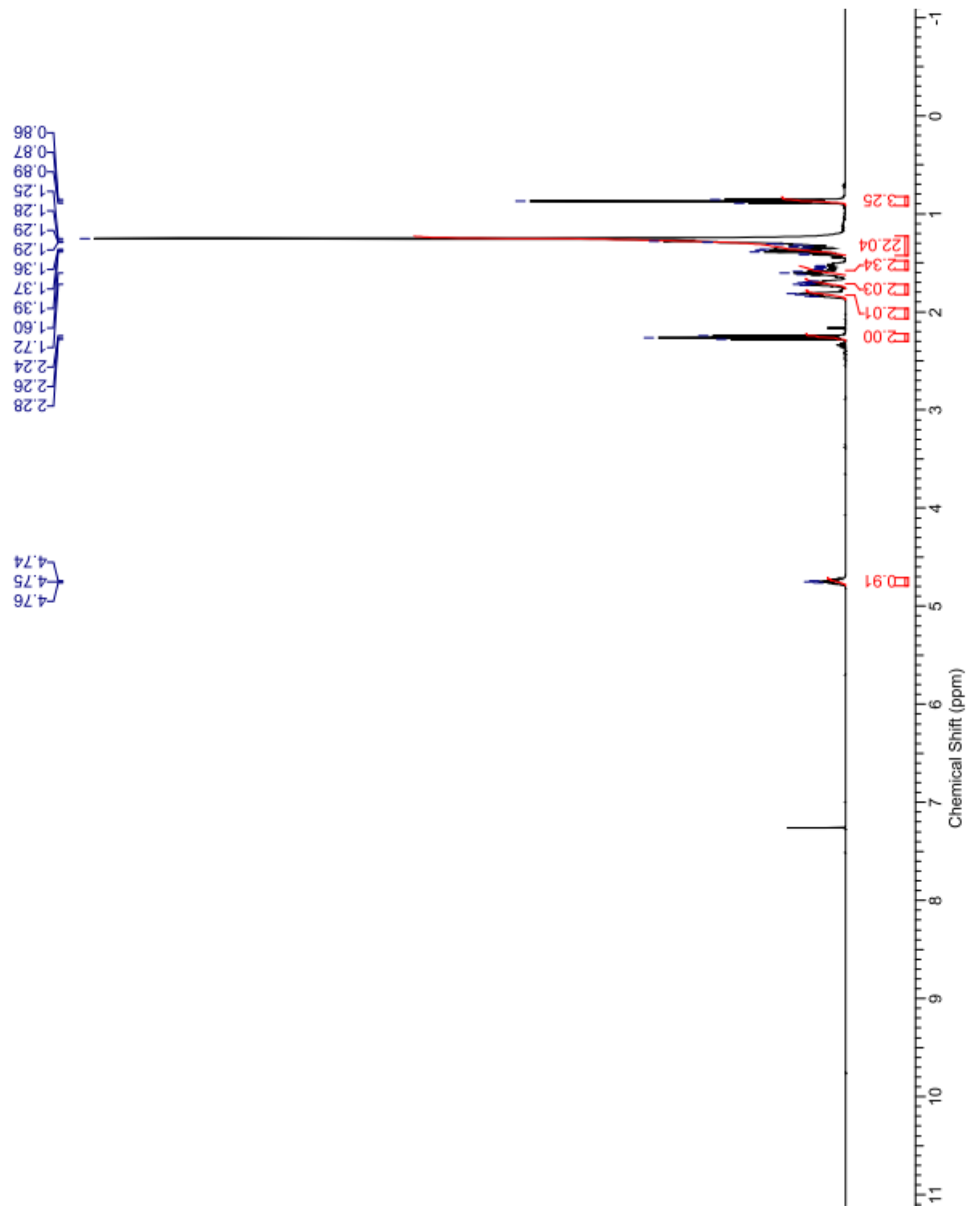



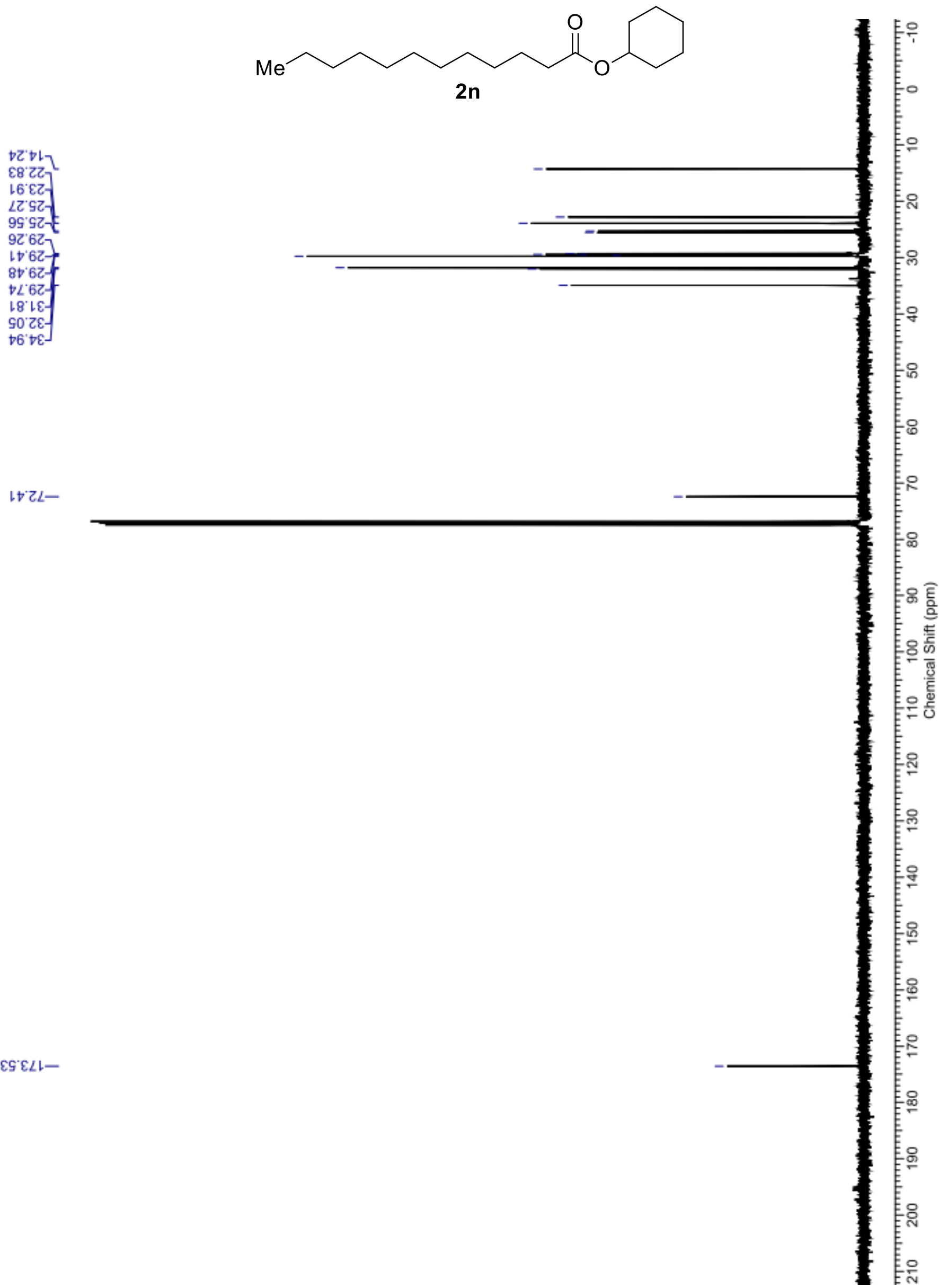

$1+Z L-$

$\varepsilon \varsigma^{\prime} \varepsilon L L-$ 
<smiles>CC(=O)OCCc1ccccc1</smiles>

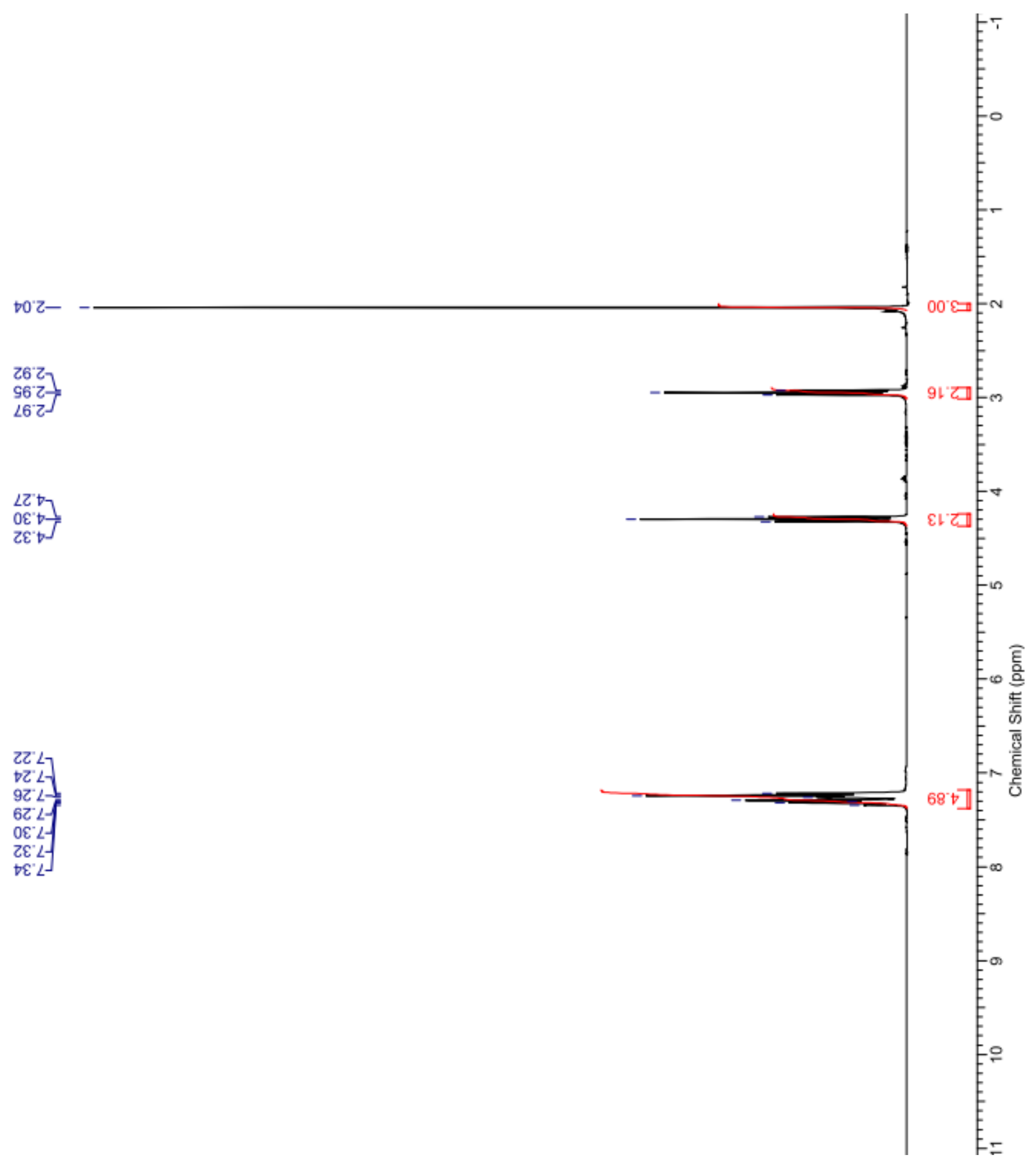




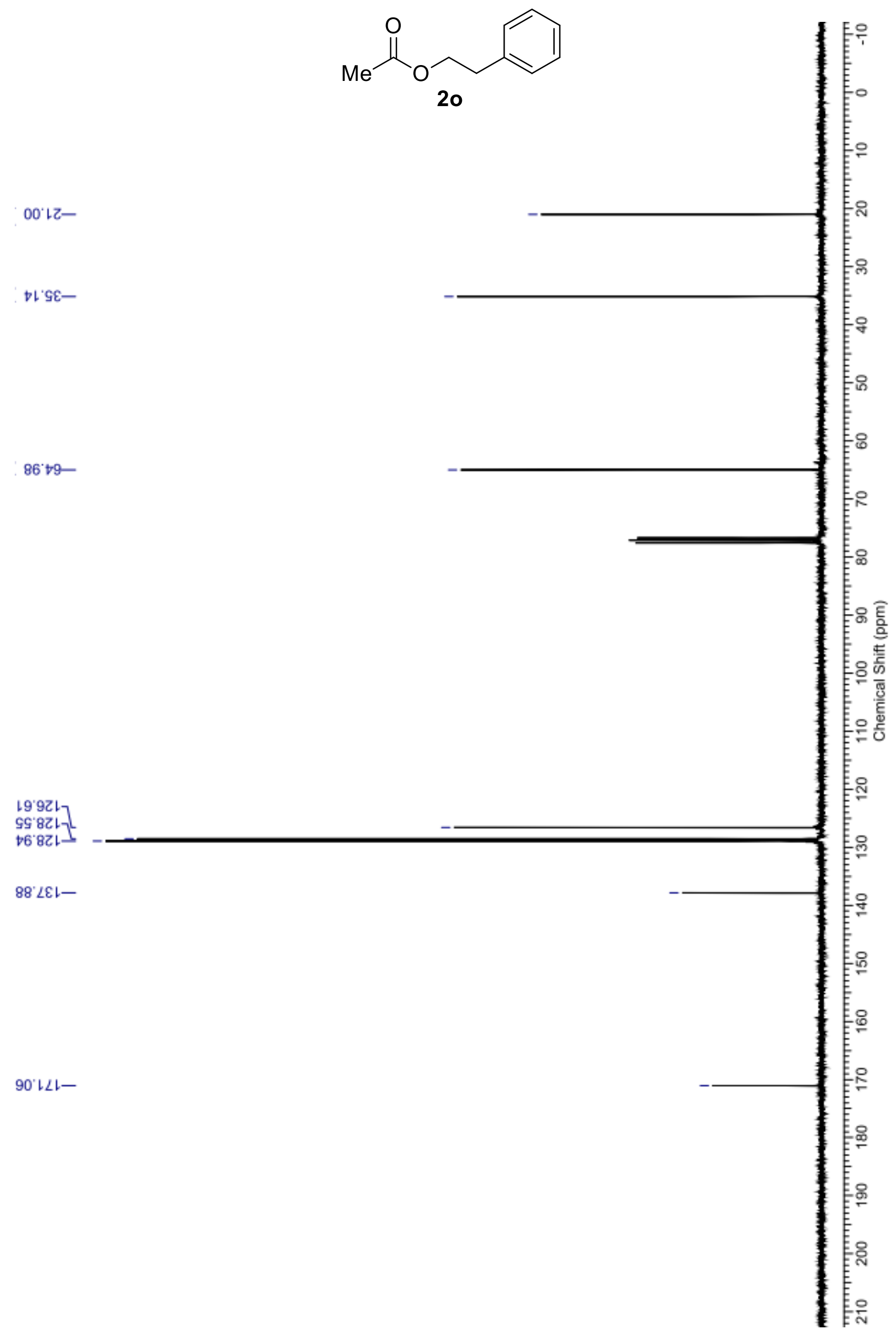




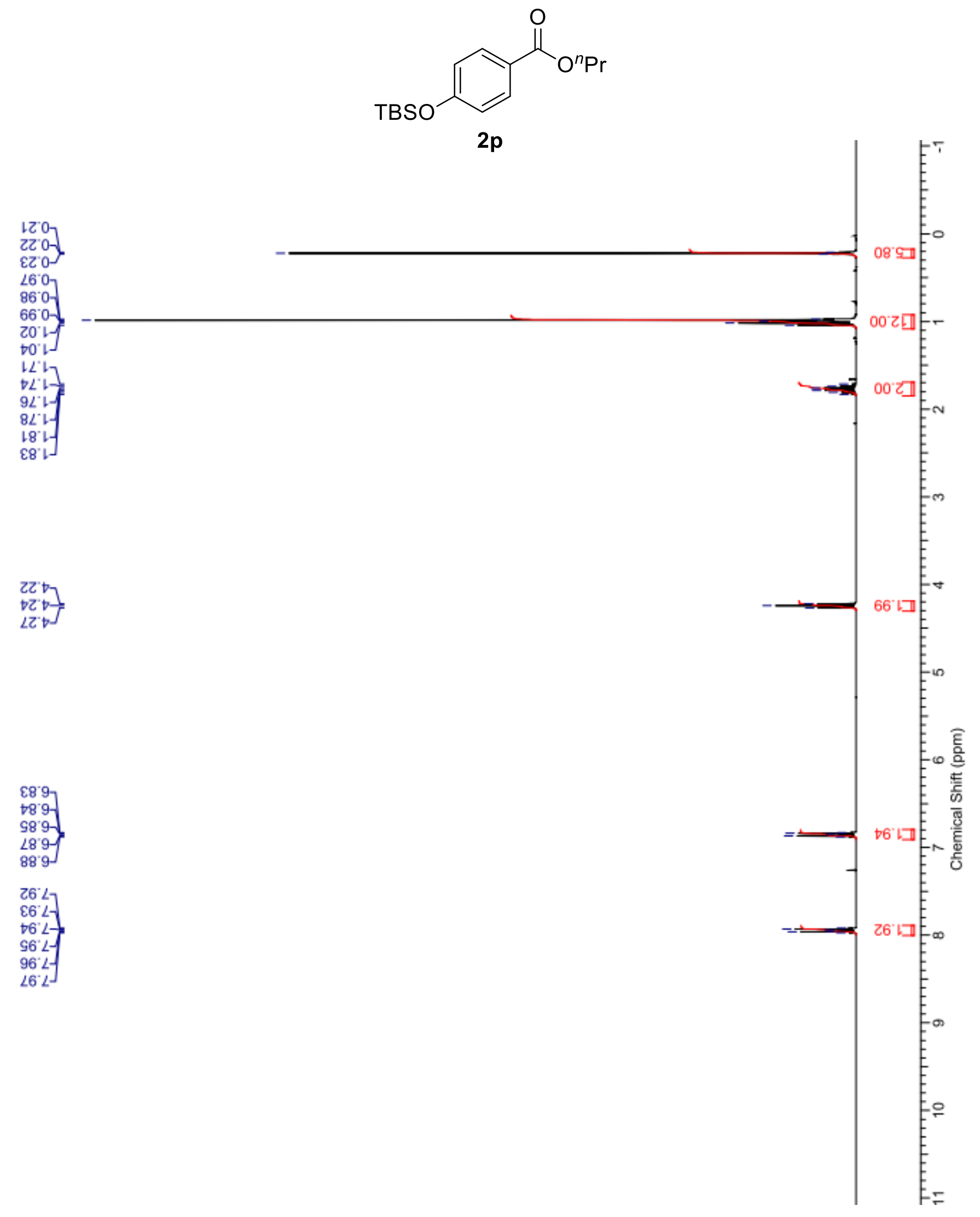




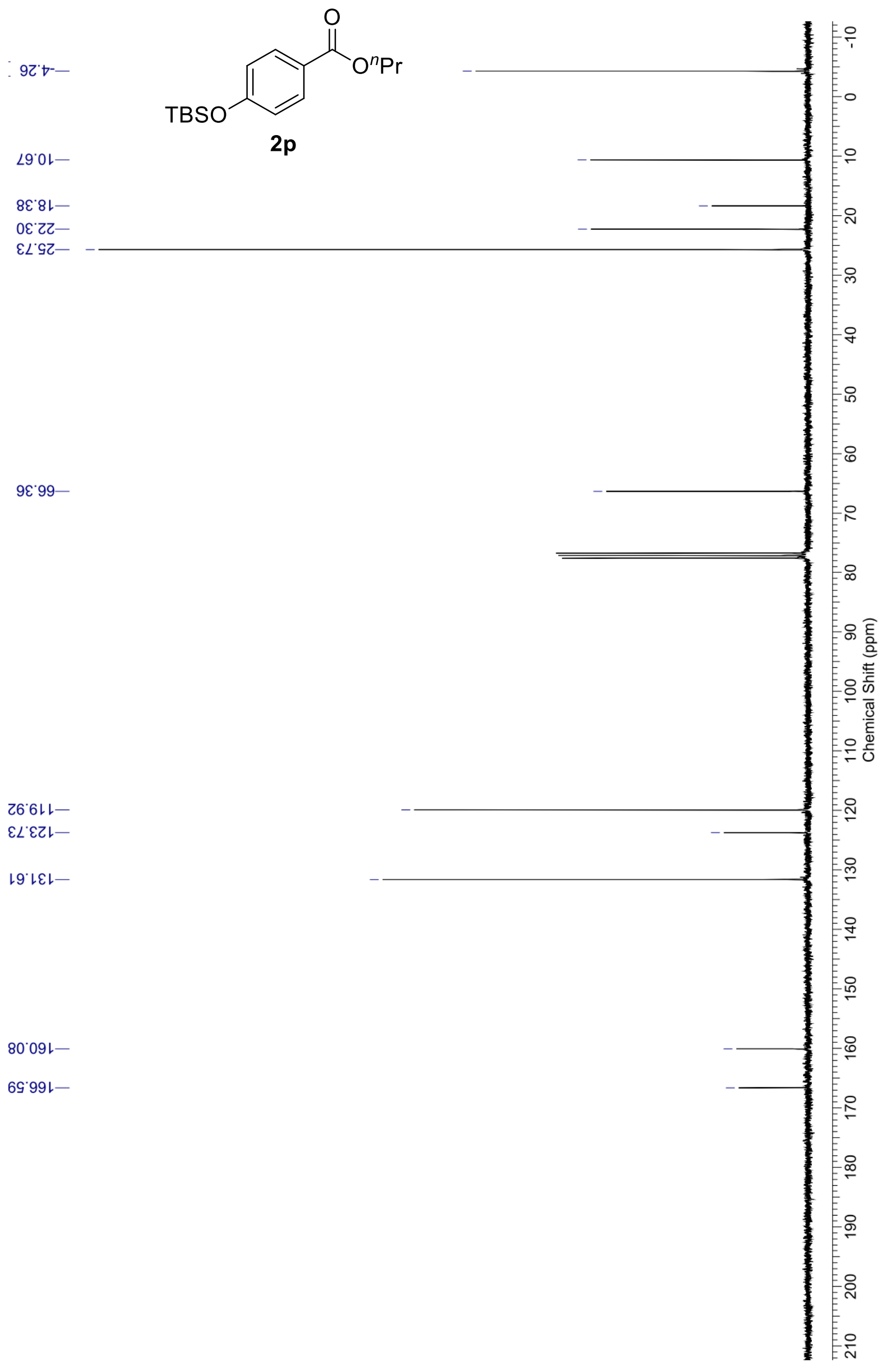




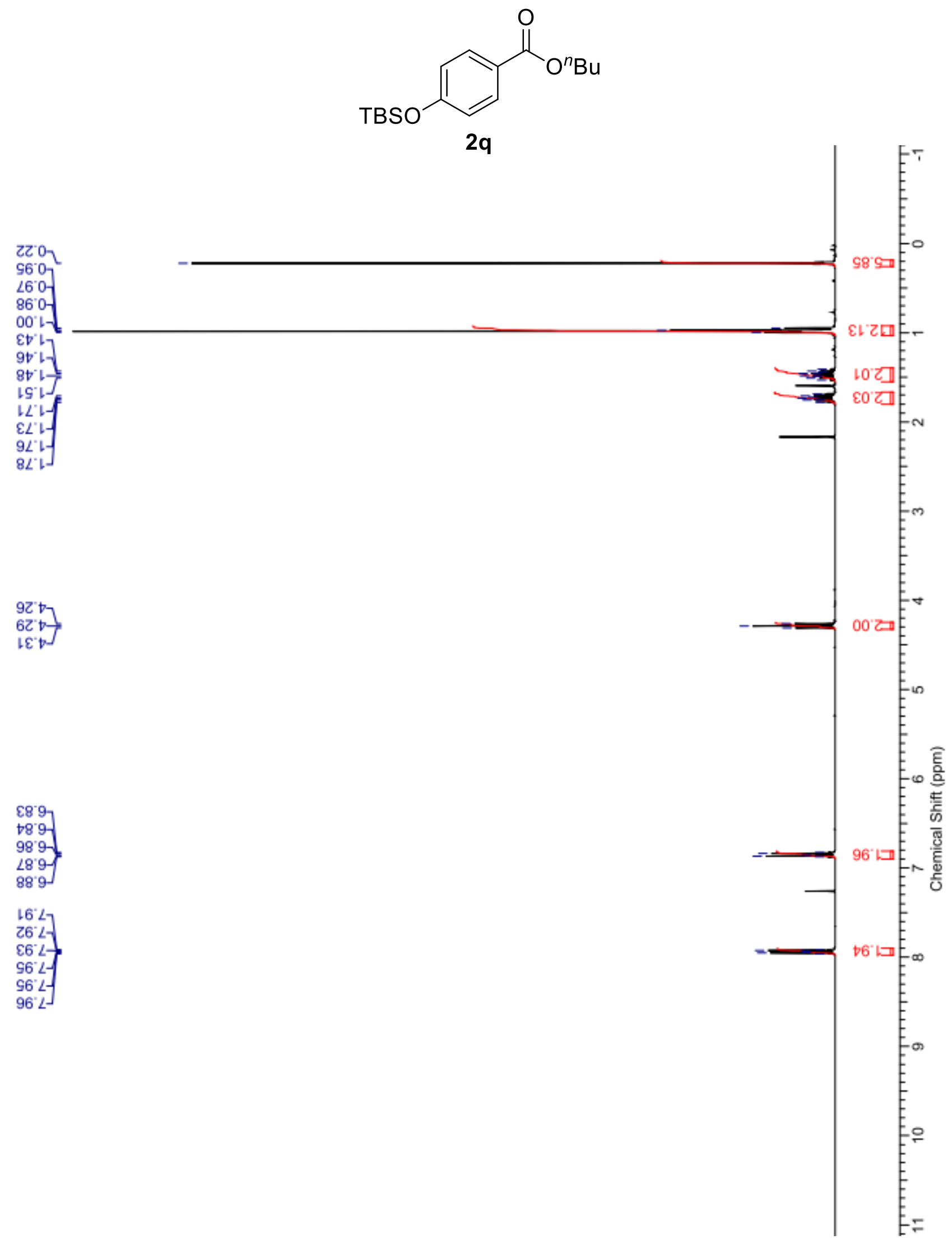




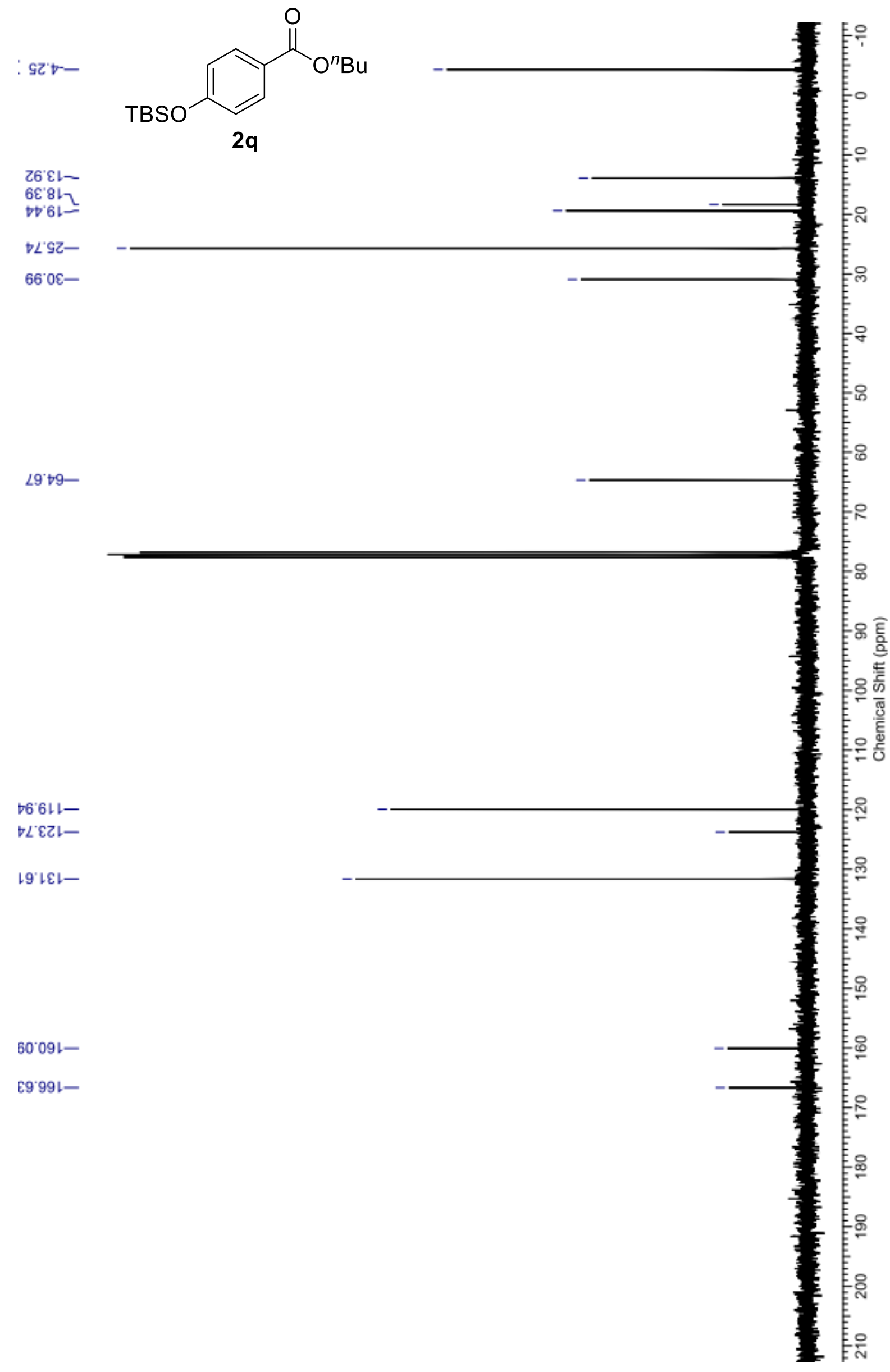




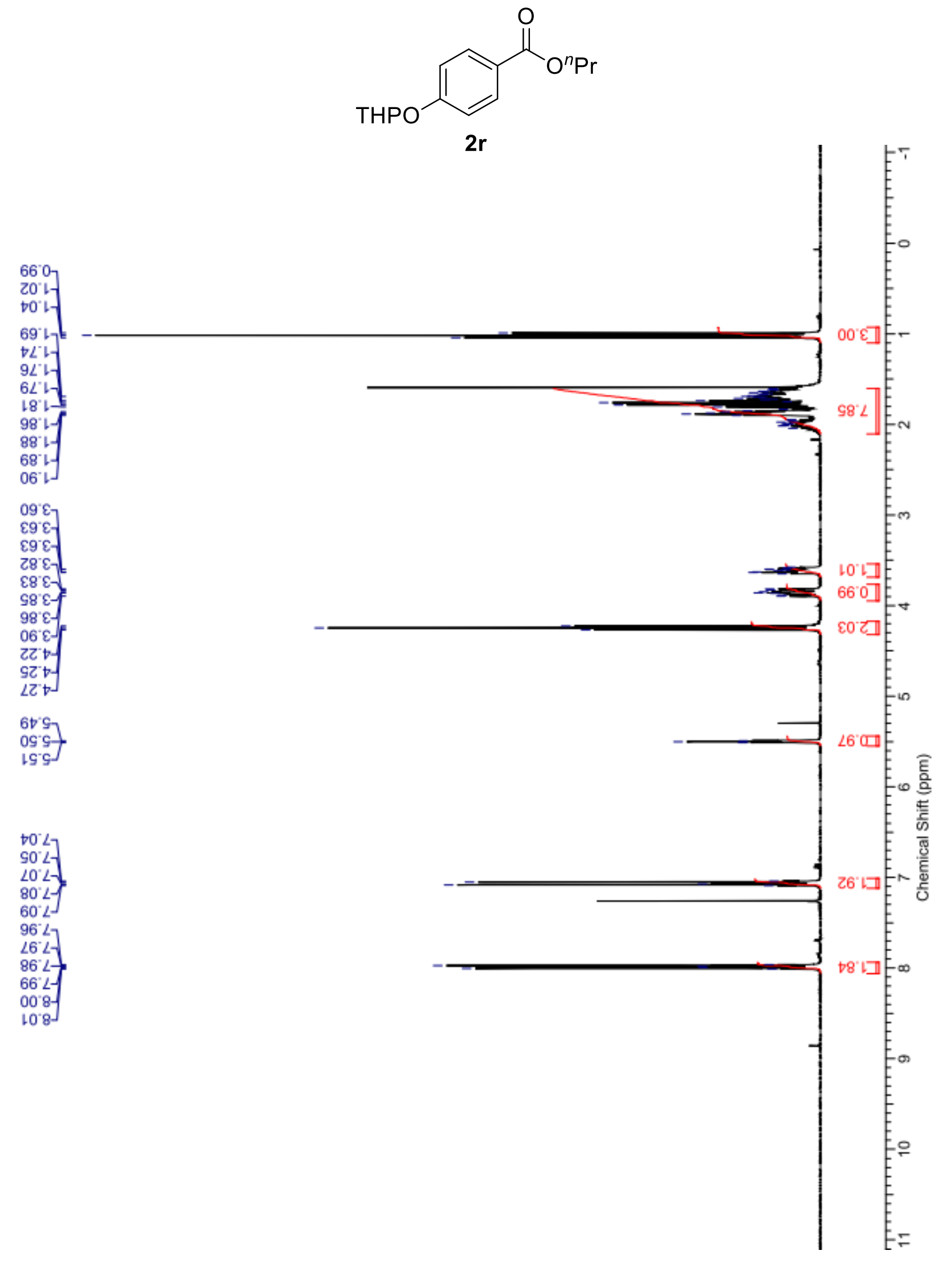


$\angle 9^{\circ} 01-$
$89.81-$
$1 \varepsilon^{\circ} Z Z-$
$2 Z^{\circ} 9 z-$
$8 Z^{\circ} 0 \varepsilon-$<smiles>CCCOC(=O)c1ccc(OP)cc1</smiles>

L1'29-

8ع 99

OZ $96-$

L6'SLL

ธิเ'ยนเ-

๑งเยเ-

96.091-

59.991-

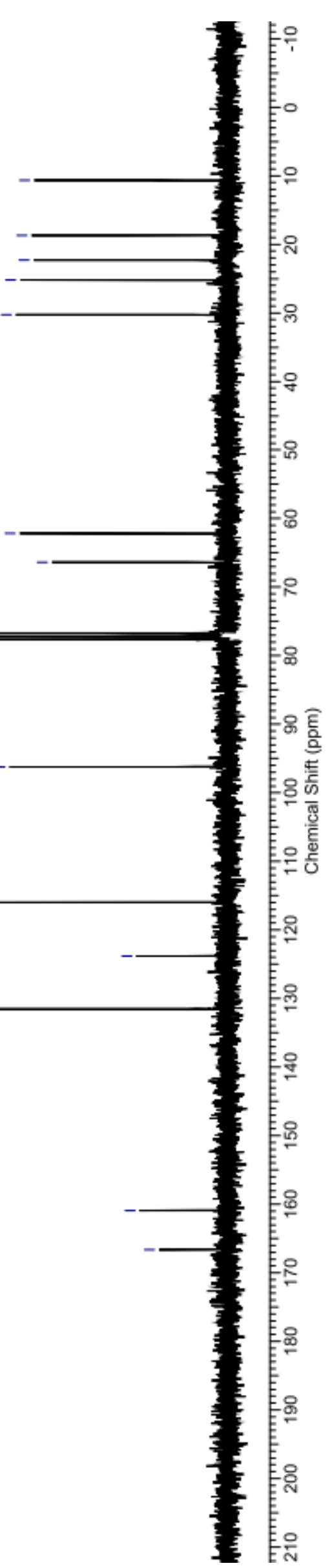




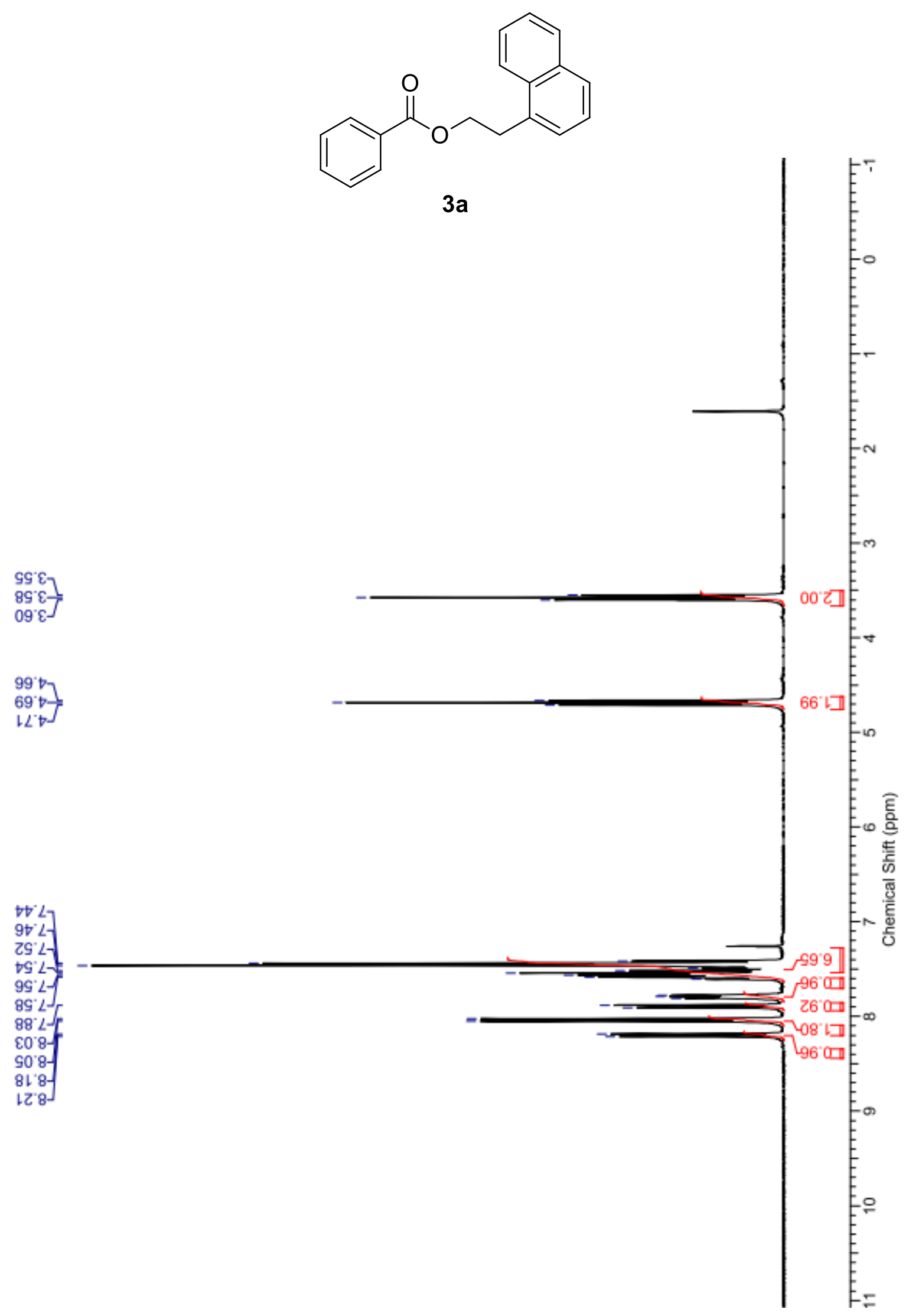




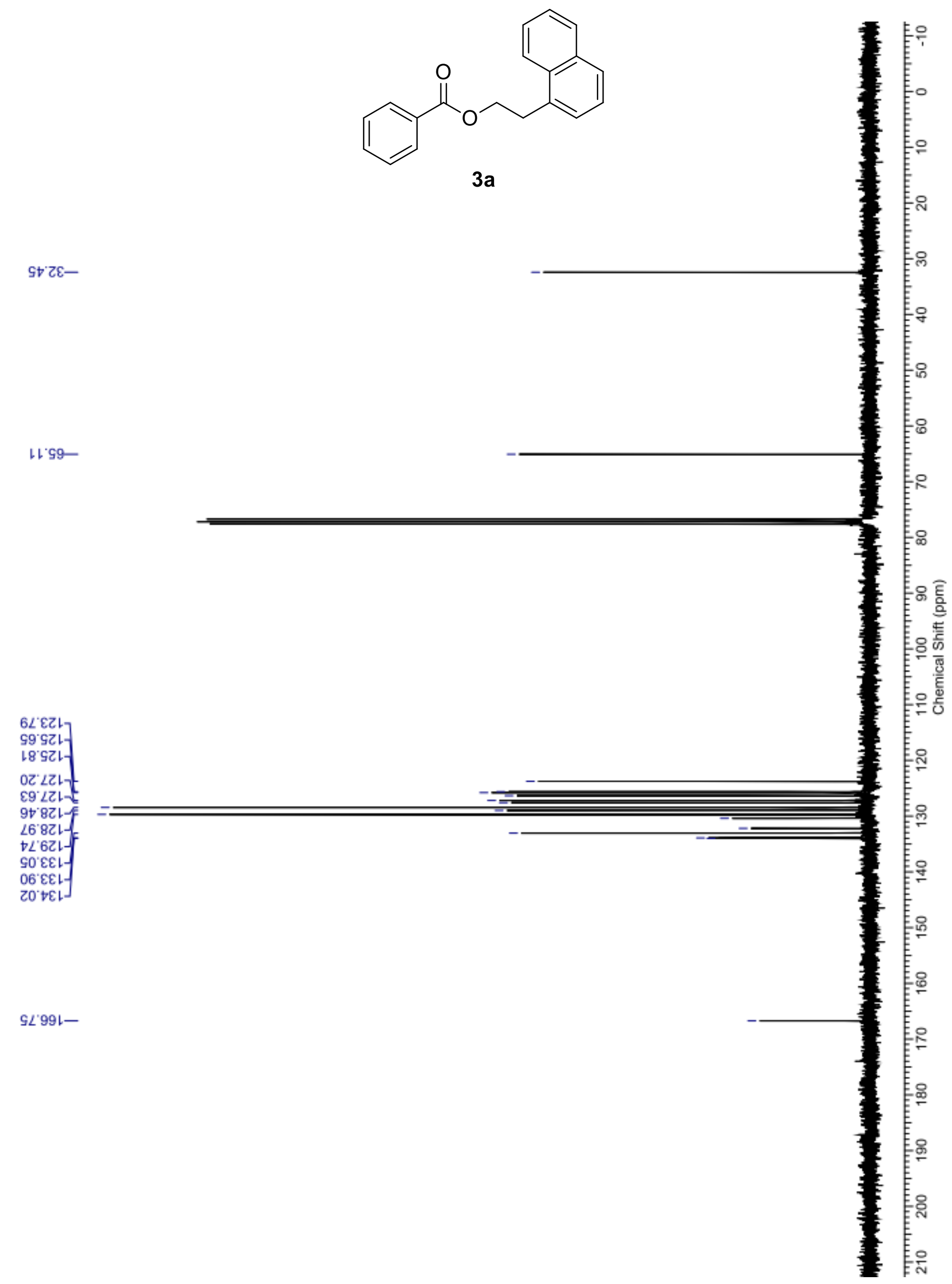




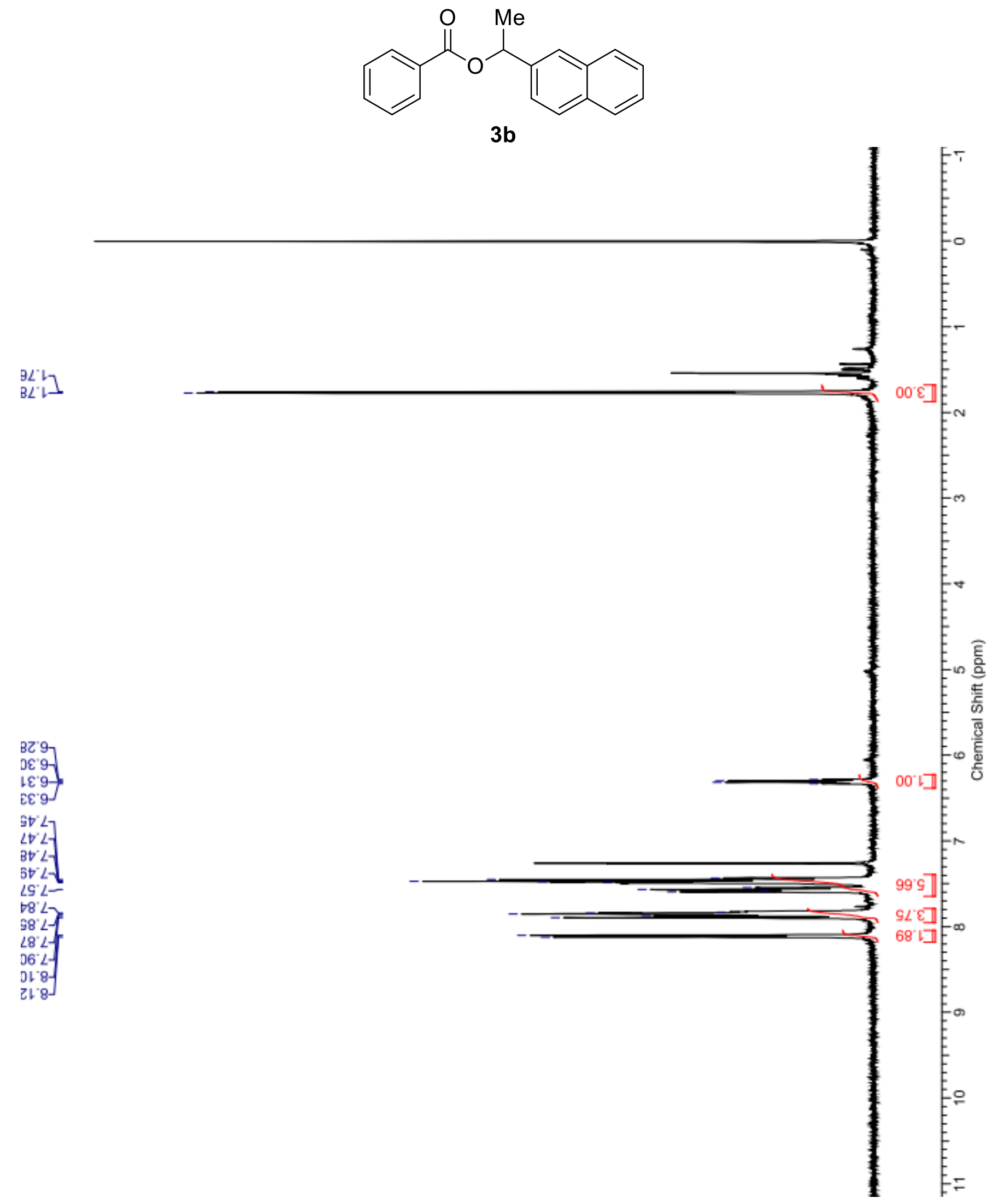




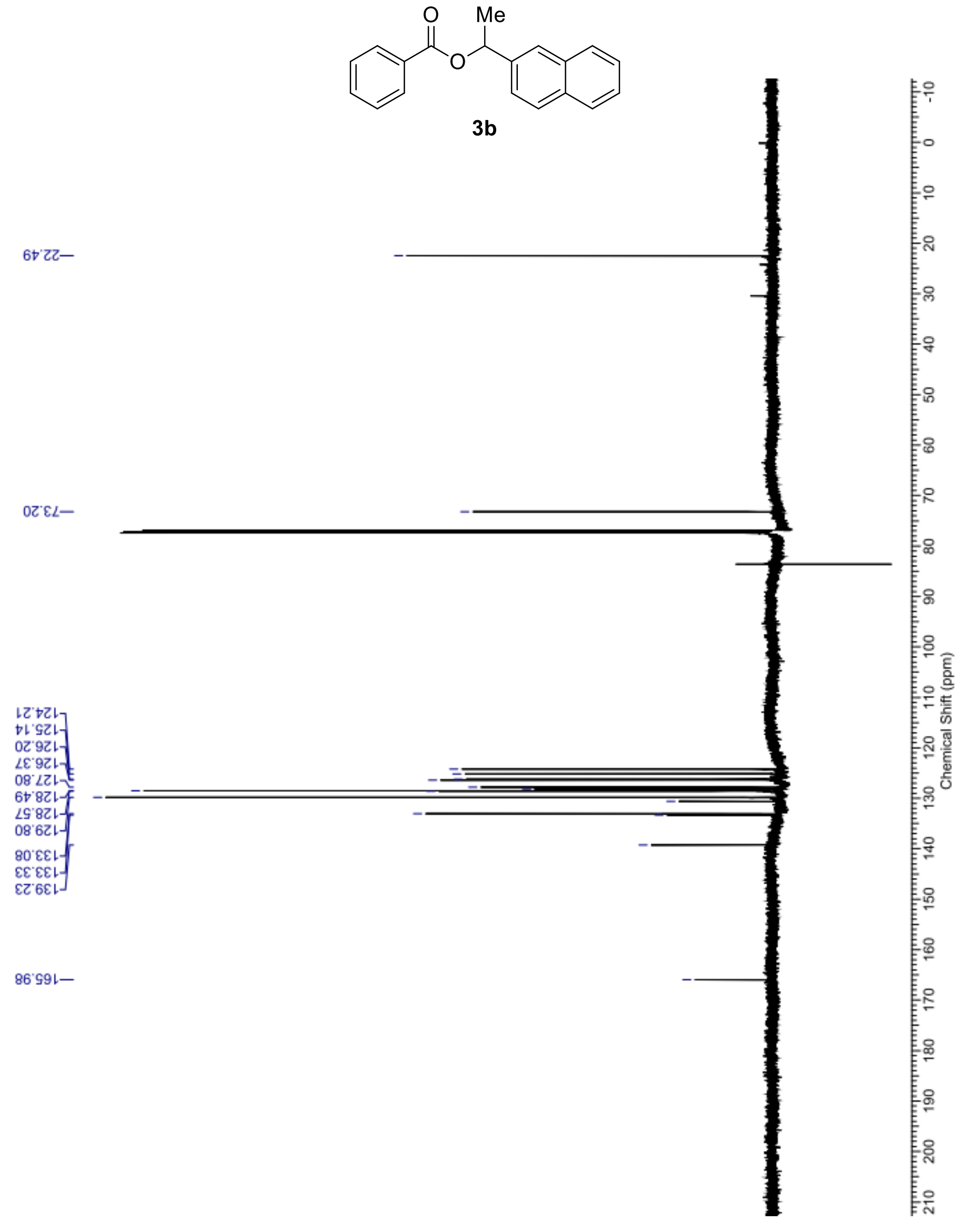




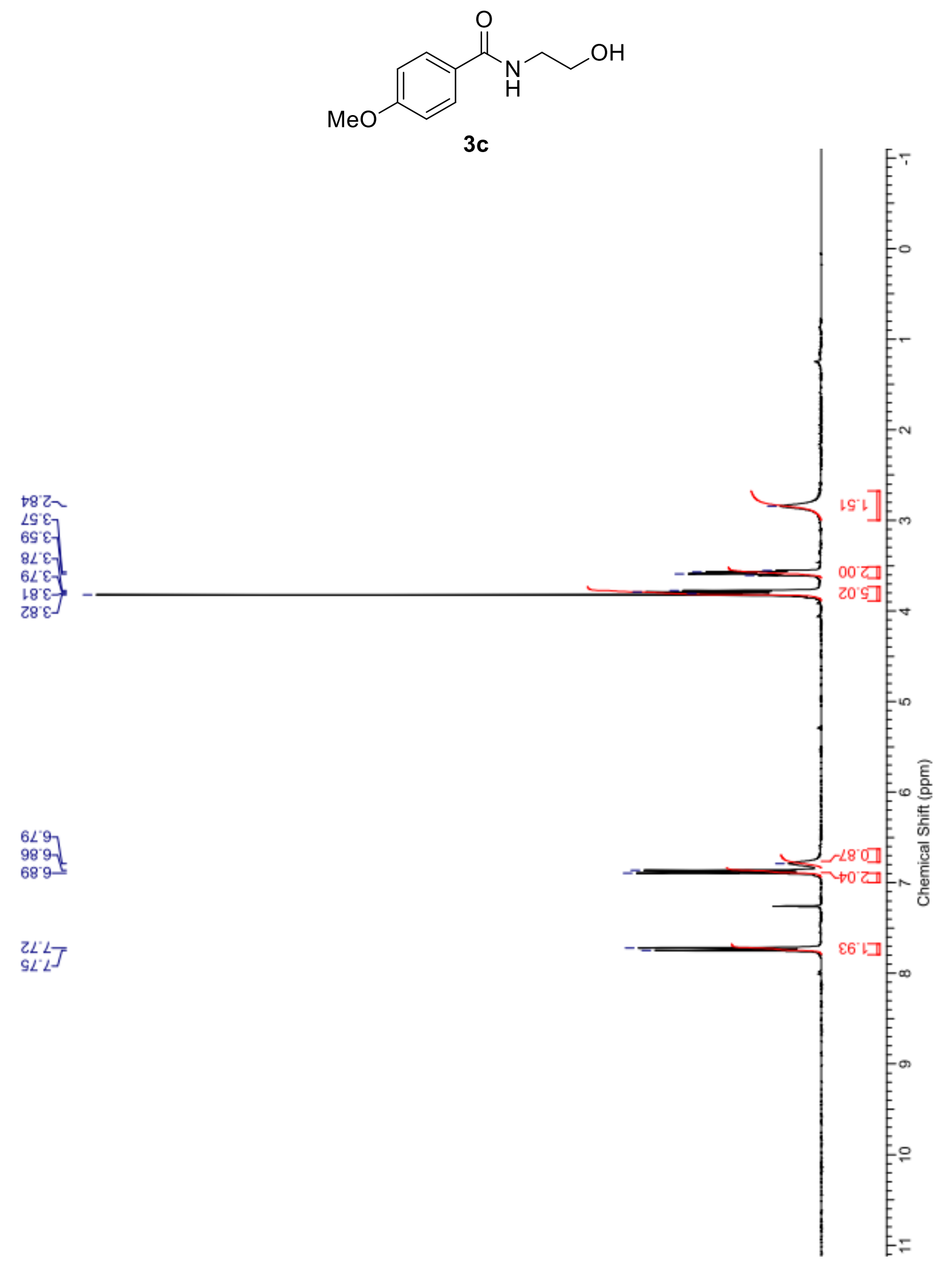




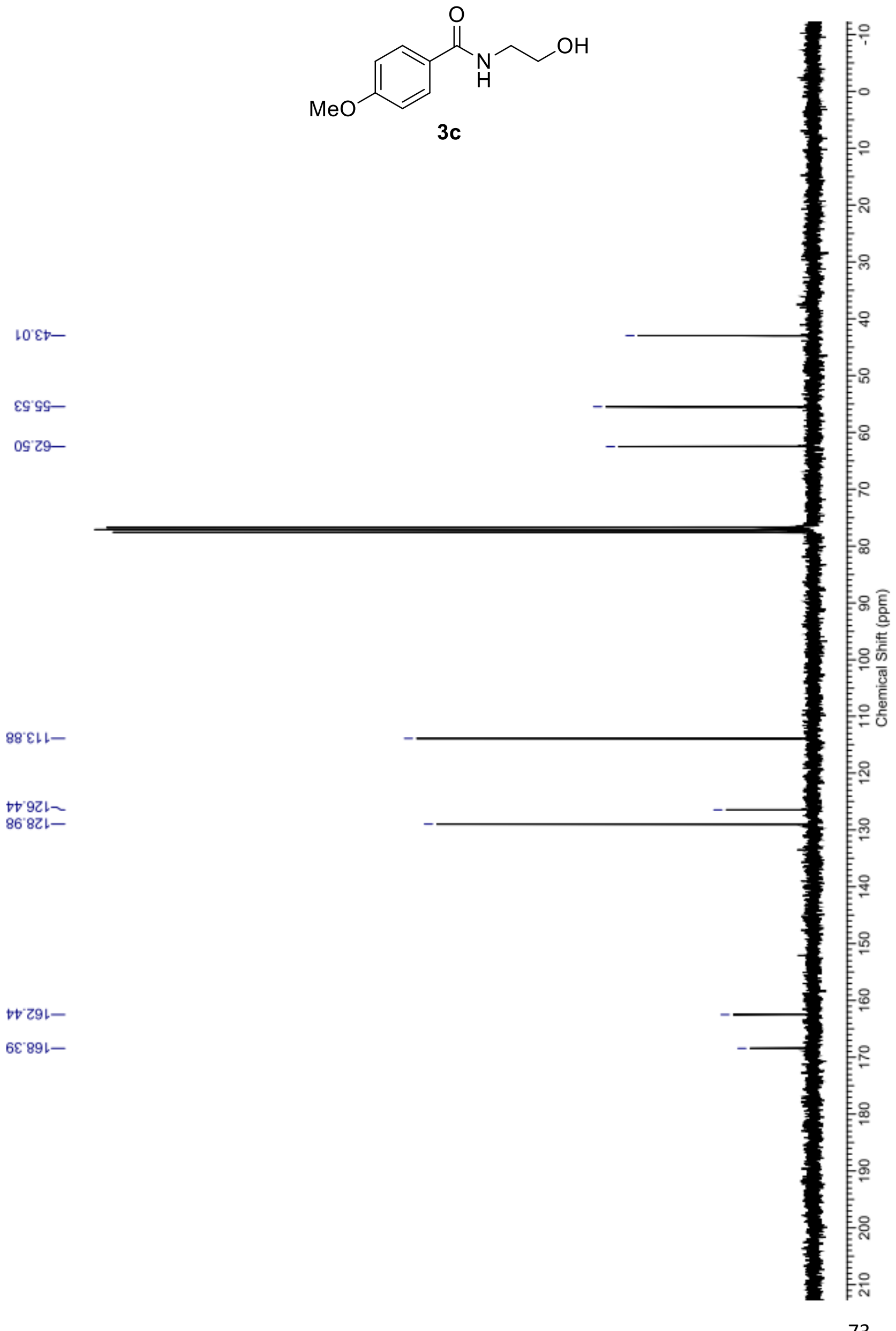




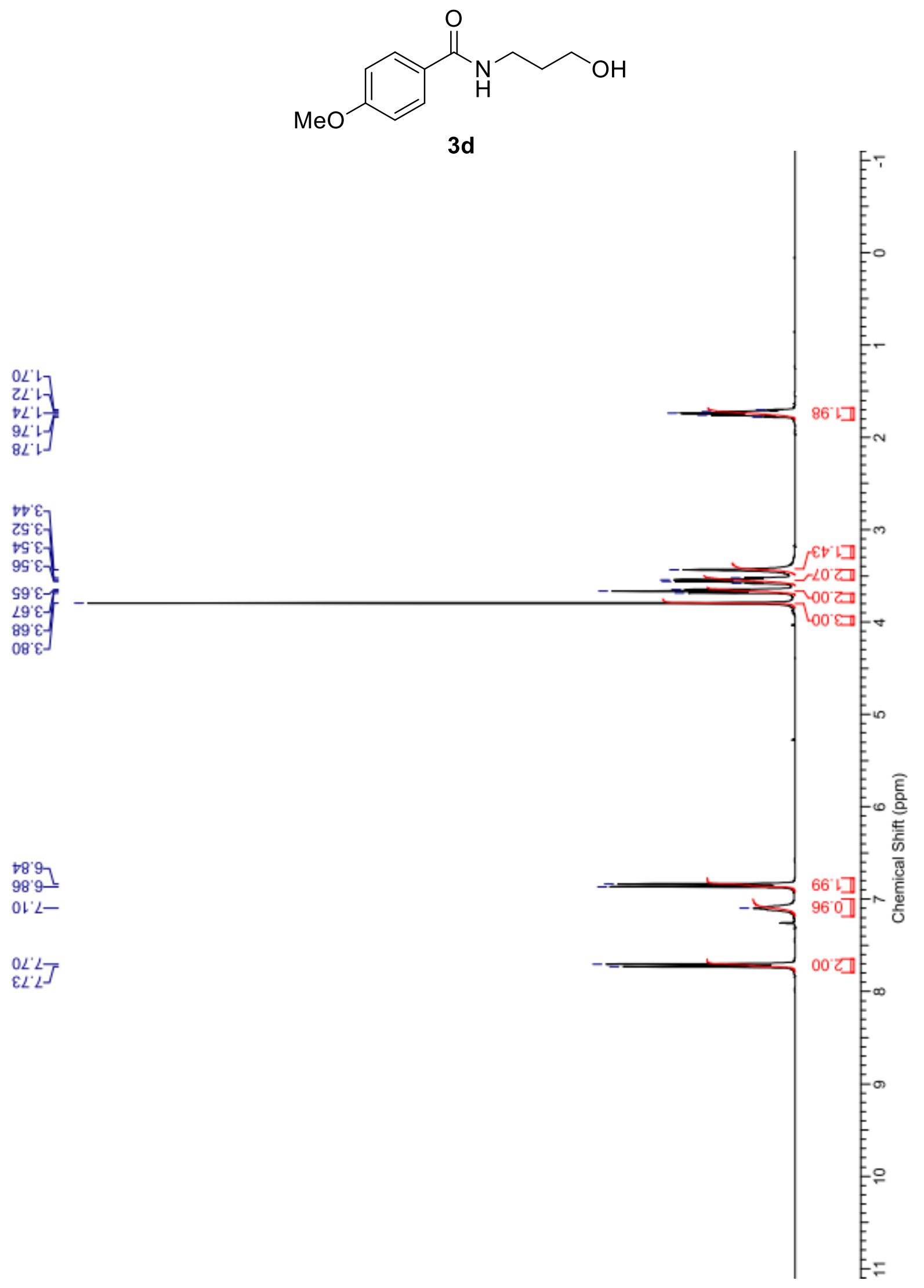




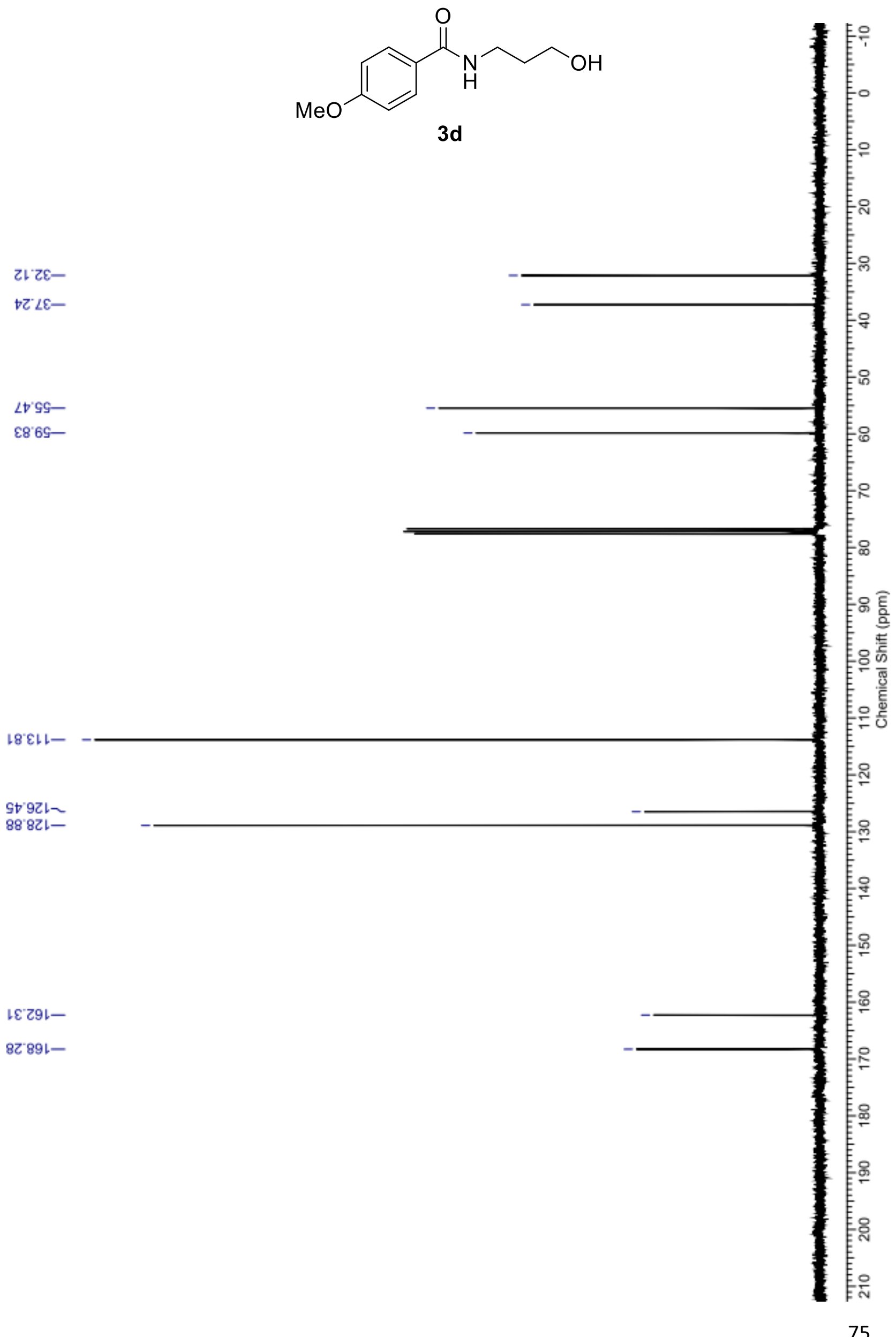



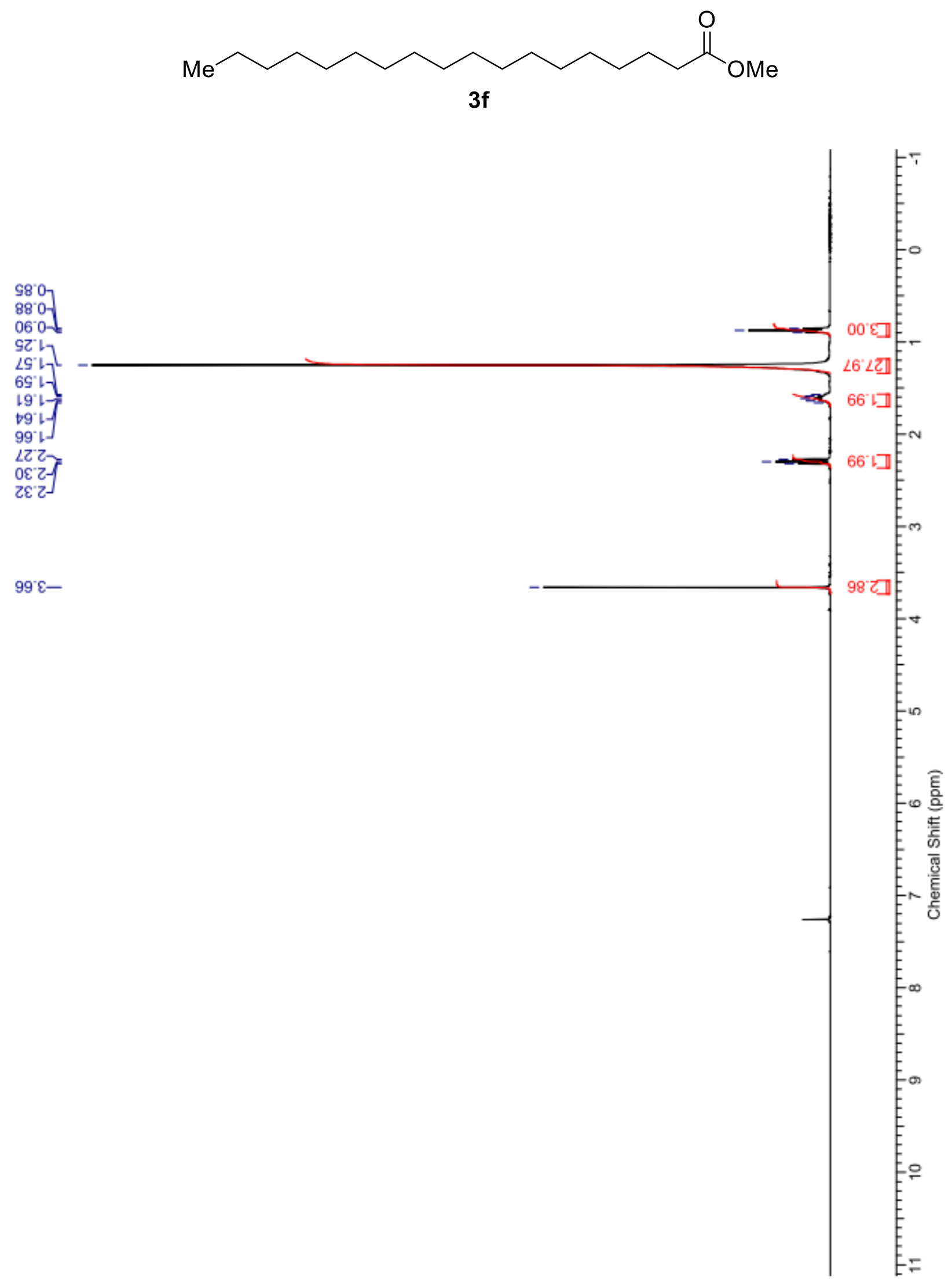


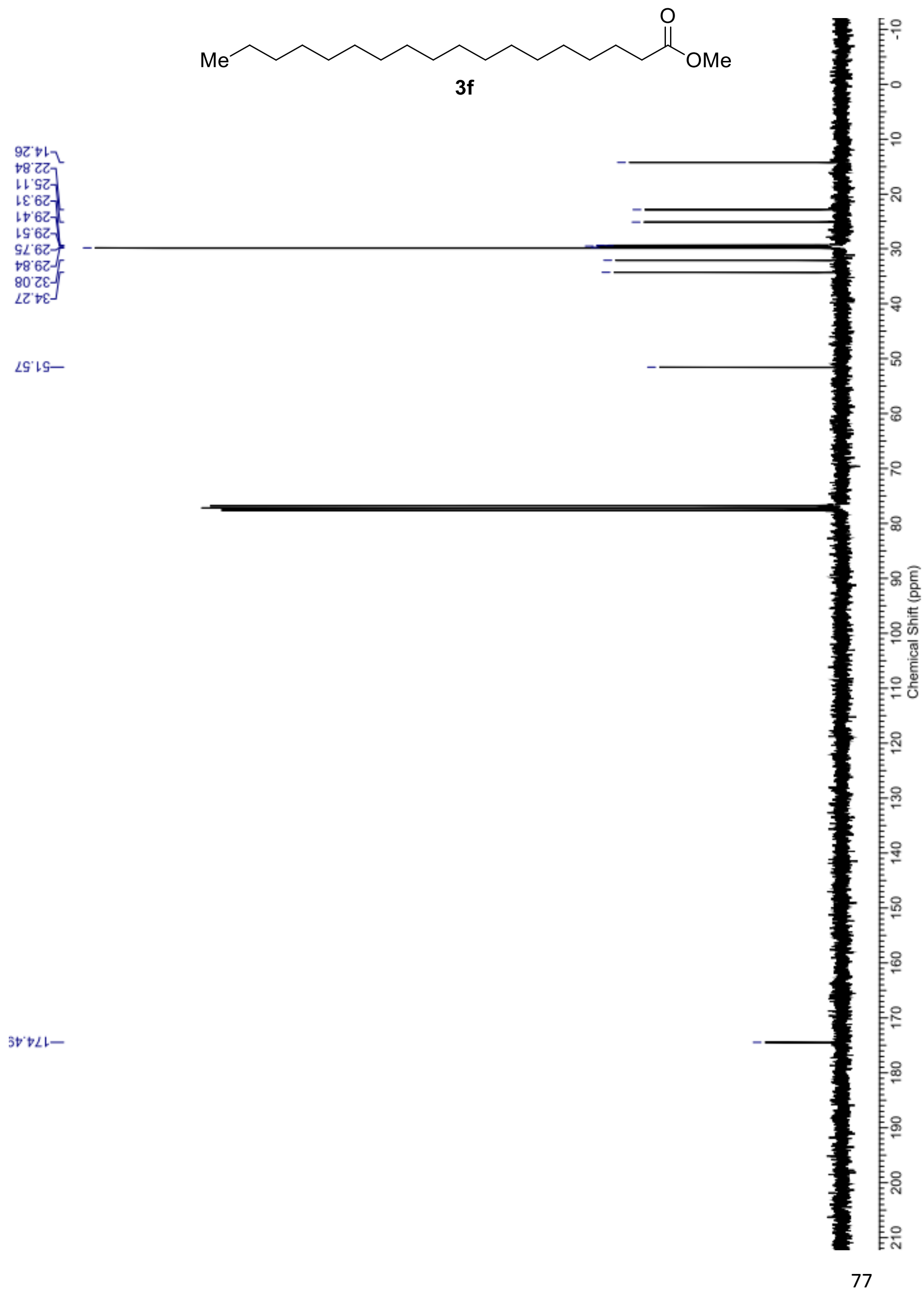


<smiles>O=C(OCCO)c1ccc(C(=O)OCCO)cc1</smiles>

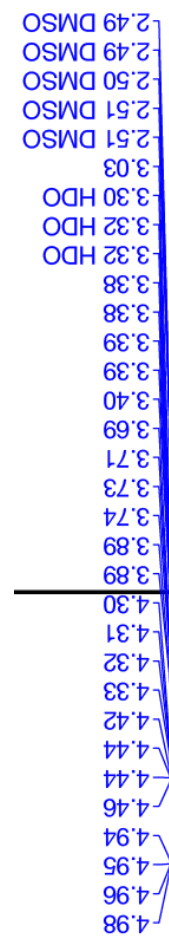

$\left.\begin{array}{l}88^{\circ} .4 \\ 80.8 \\ 60.8 \\ 01.8 \\ 21^{\circ} \cdot 8 \\ 21^{\circ} \cdot 8\end{array}\right]$ 


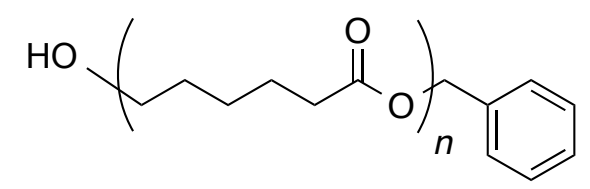

Polycaprolactone (PCL)

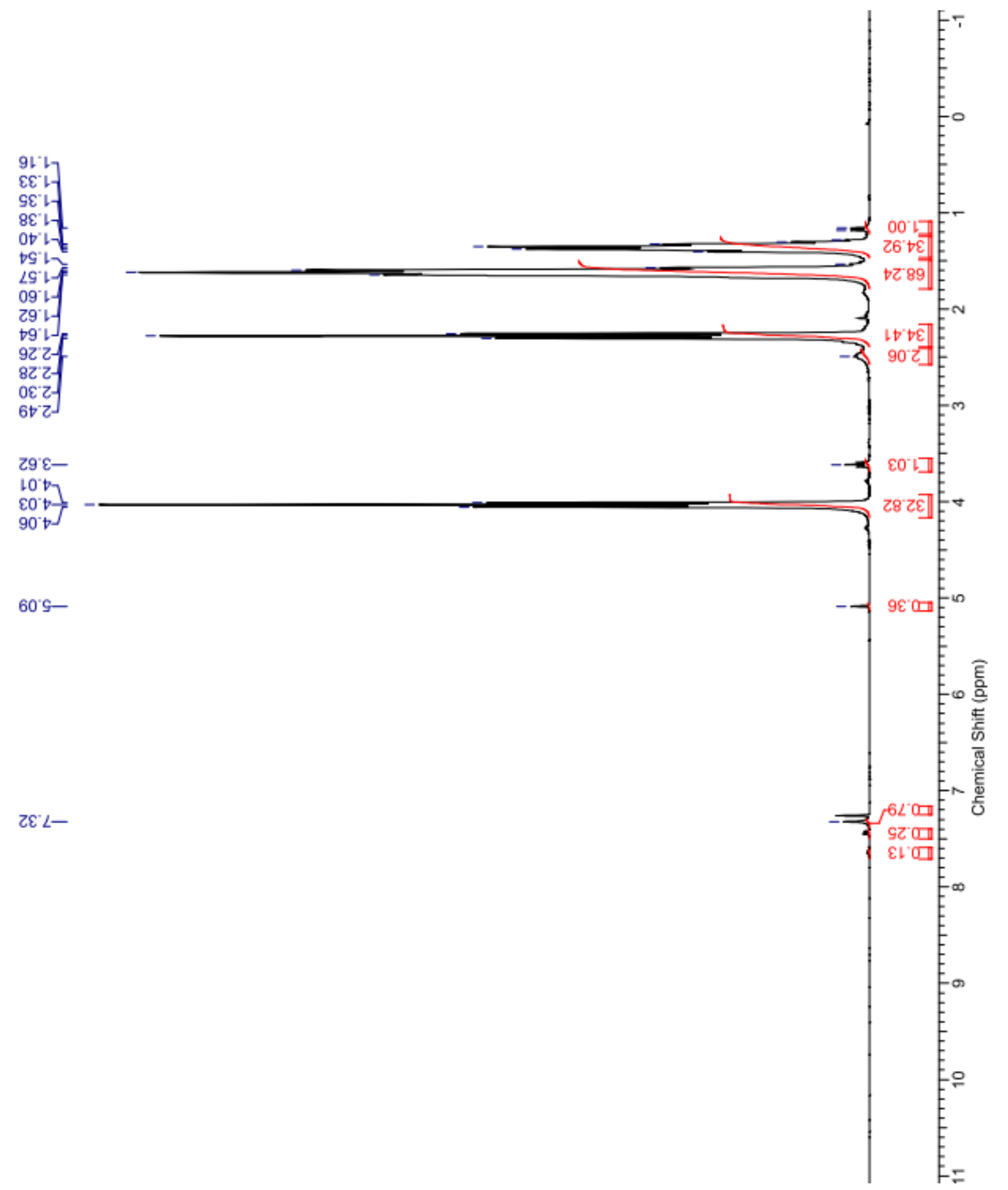




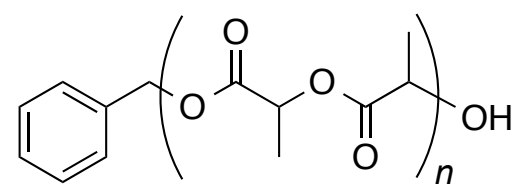

Polylactide (PLA)

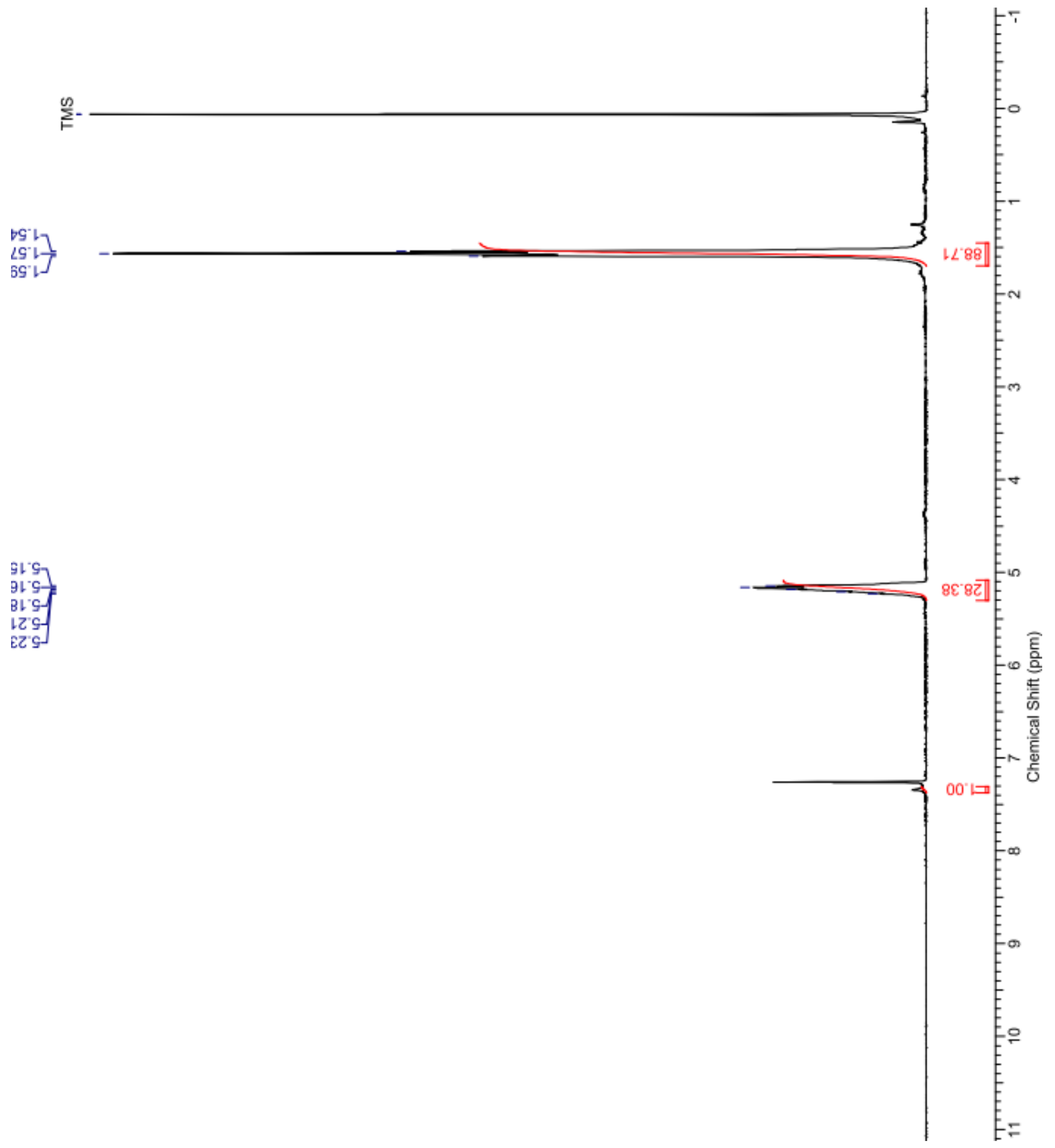

